\title{
SENSITIVITY INVESTIGATION OF EQUILIBRIUM STATES AND THE DETERMINATION OF THE FOLD LINE OF LIMIT POINTS FOR AN ELASTIC BODY
}

\author{
IMRE KOZÁK ${ }^{1}$ \\ Institute of Applied Mechanics, University of Miskolc \\ H-3515 Miskolc-Egyetemváros
}

[Received: November 4, 2016; Accepted: January 18, 2017]

\begin{abstract}
The paper presents the stability theory of a 3D elastic body by setting up those equations which govern the problem in the frameworks of the finite element method. In this respect it appears partly as a review paper. Special emphasis is laid on the issue, of how to determine the fold line of the limit points.
\end{abstract}

Mathematical Subject Classification: 74G60, 74B15

Keywords: Finite deformation, follower loads, imperfections, critical points, fold line

\section{INTRODUCTORY REMARKS}

A number of books have been published on the problem of stability. The first one [1] appeared in 1936. The fast development of the solution methods and deeper clarification of the theoretical background began in the sixties of the last century. In this respect a pioneering work was book [2] by Bolotin in 1964. As regards the theoretical background and the correct mathematical formulation of the stability problem it is worth referring to books [3 8] and [9]. Various numerical methods can be used to find the equilibrium path and the characteristic points (a limit point for example) on it. One of them is the finite element method (FEM) - see for instance [10 or as an example for the application of FEM the papers [11, 12. Though we have just cited some books it is also inevitable here to refer to Subsection 68bis. in Trusdell and Noll [13]: 'There is a vast literature on elastic stability and to little purpose. Most of it rests on upon improper, or at best unduly special formulation of the principles of elasticity. Whole volumes have been devoted to presenting ostensible solutions to particular problems by means of criteria that are never even clearly stated and morass of equations spouted forth on the subject can be regarded as little else then rhetoric. Stability theory is, necessarily, an application of some theory of finite deformations, such as elasticity, but most of the specialists of stability theory show no evidence of having troubled to learn the theory they claim to be applying.'

\footnotetext{
1 The author died in 2016. The present paper is his last work which he could not totally complete.
} 
In the present paper a stability investigation of an elastic body is carried out the basis of a continuum mechanical model in which the problem is considered as a 3D one by using a static procedure applying the nonlinear theory of deformation. It is assumed that the constitutive relation between the Green-Lagrange deformation tensor and the second Piola-Kirchoff stress tensor is a linear one. The load the body is subjected to is a surface load which is always perpendicular to the deformed surface and is, therefore, in general non-conservative.

A difference is made between the stress and deformation free state of the body (initial configuration) and the instantaneous equilibrated states (current configurations).

The perfect state of the body and the deviations from the perfect state are also treated. Deviations might be caused by load imperfections and/or geometrical imperfections. The imperfections in the problems considered are described by imperfection parameters.

The problems outlined in the title of the present paper include the following problems:

- finding the equilibrium states (configurations) and depicting the equilibrium paths and surfaces using the parameters of the problem considered;

- calculation of the work done by the total force system of the equilibrium state on the equilibrium path and setting up stability criteria;

- qualifying the critical points (bifurcation and limit points) on the equilibrium paths;

- determination of the fold line by a total stability analysis (joining the limit points of the equilibrium paths) or directly (departing from a limit point state).

In the present paper the equations of the 3D continuum mechanical model are affiliated with those of the finite element model.

Arbitrary curvilinear coordinate systems are assumed for the continuum mechanical models. In direct notation the (vectors) [tensors] are denoted by (boldface) [italic boldface] letters. The components of such quantities are denoted by the same letter in lightface with indices identifying the component considered. The indices have the range 1,2,3 and the subscripts preceded by a semicolon denote covariant derivations with respect to the coordinates that belong to the subscripts.

For the finite element models the matrices are denoted by boldface letters and the number of indices shows the size of the matrix. The indices have the range $1,2, \ldots, m$ where $m$ is the degree of freedom. Transposition is denoted by ()$^{\mathrm{T}}$.

For dummy indices the summation convention should be applied both in the equations of the continuum model and in the equations of the finite element model.

\section{Assumptions and notational CONVEntions}

The initial configuration and the instantaneous equilibrium configurations (current configurations) are designated by $(B)$ and $(\bar{B})$ or $(\bar{B}+\Delta \bar{B})$.

Quantities in the current configuration are denoted by barred letters. However there is no a distinguishing symbol (bar) for the same quantities in the initial configuration. 
Displacement and stress boundary conditions are assumed. The displacement boundary conditions are independent of the problem parameters and the deformations of the body.

Explicating the train of thought is performed in the Lagrangian description, i.e., the tensor fields that describe the physical state of the body are considered in state $(B)$ of the body. In this configuration $\left(x^{1}, x^{2}, x^{3}\right)$ is an arbitrary curvilinear coordinate system for which $\mathbf{g}_{k}$ and $\mathbf{g}^{l}$ are the base vectors, $g_{k l}$ is the metric tensor, $g=\operatorname{det} g_{k l}$ and $\delta_{k}^{l}$ is the Kronecker delta.

In configuration $(B)$ of the body:

$$
\mathbf{u}=u^{k} \mathbf{g}_{k}, \quad \Delta u^{k}, \quad \delta u^{k}=\delta\left(\Delta u^{k}\right)
$$

are the displacement vector, its increment and the virtual displacement. The GreenLagrange strain tensor and its increment are given by

$$
\begin{gathered}
E_{k l}=\frac{1}{2}\left(u_{k ; l}+u_{l ; k}+u_{; k}^{m} u_{m ; l}\right), \\
\Delta E_{k l}=\Delta E_{k l}^{(1)}+\Delta E_{k l}^{(2)}
\end{gathered}
$$

where the number in parentheses shows what the power of the derivatives is in the quantities considered - see for instance relations 2.6 and 2.7.

The constitutive equations in configuration $(B)$ of the body, which is assumed to be homogeneous, relate the second Piola-Kirchoff stress tensor (called simply stress tensor) to the Green-Lagrange strain tensor

$$
S^{p q}=E_{k l} C^{k l p q}=C^{p q k l} E_{k l} .
$$

where $C^{k l p q}$ is the tensor of material constants (stiffness tensor).

The increment of the stress tensor is given by

$$
\Delta S^{p q}=\Delta E_{k l} C^{k l p q}=\Delta E_{k l}^{(1)} C^{k l p q}+\Delta E_{k l}^{(2)} C^{k l p q}
$$

in which according to equation $(2.2)$

$$
\begin{aligned}
& \Delta E_{k l}^{(1)} C^{k l p q}=\Delta u_{m ; k}\left(\delta_{l}^{m}+u_{; l}^{m}\right) C^{k l p q}, \\
& \Delta E_{k l}^{(2)} C^{k l p q}=\frac{1}{2} \Delta u_{m ; k} \Delta u_{; l}^{m} C^{k l p q} .
\end{aligned}
$$

Let

$$
\mathrm{d} \overline{\mathbf{A}}=\mathrm{d} \bar{A}_{\bar{s}} \overline{\mathbf{g}}^{s}=\mathrm{d} \bar{A}_{k} \mathbf{g}^{k}
$$

be the surface element vector in the current configuration $(\bar{B})$ where $\mathrm{d} \bar{A}_{k}$ denotes the components of the vector $\mathrm{d} \overline{\mathbf{A}}$ in the local basis $\mathbf{g}^{k}$, i.e., in the initial configuration. Further let

$$
\mathrm{d} \mathbf{A}=\mathrm{d} A_{p} \mathbf{g}^{p}
$$

be the surface element vector in the initial configuration. As is well known

$$
\mathrm{d} \bar{A}_{k}=Q_{k}^{p} \mathrm{~d} A_{p}
$$

where

$$
Q_{k}{ }^{p}=\frac{1}{2} e_{k l m} e^{p q r}\left(\delta_{q}^{l}+u_{; q}^{l}\right)\left(\delta_{r}^{m}+u_{; r}^{m}\right)
$$


while $e_{k l m}$ and $e^{p q r}$ are the permutation symbols.

The increment of the surface element vector of configuration $(\bar{B})$ in the local coordinate system of the initial configuration (in state $(B)$ ) is given by:

$$
\Delta\left(\mathrm{d} \bar{A}_{k}\right)=\Delta Q_{k}^{p} \mathrm{~d} A_{p}=\Delta Q_{k}^{(1)} p \mathrm{~d} A_{p}+\Delta Q_{k}^{(2)} p \mathrm{~d} A_{p},
$$

where

$$
\Delta Q_{k}^{(1) p}=e_{k l m} e^{p q r}\left(\delta_{q}^{l}+u_{; q}^{l}\right) \Delta u_{; r}^{m}, \quad \Delta Q_{k}^{(2) p}=\frac{1}{2} e_{k l m} e^{p q r} \Delta u_{; q}^{l} \Delta u_{; r}^{m} .
$$

The position vector is denoted by $\mathbf{r}$.

Parts $\bar{A}_{\mathrm{tI}}$ and $\bar{A}_{\mathrm{tII}}\left(\bar{A}_{\mathrm{tI}} \cap \bar{A}_{\mathrm{tII}}=0\right)$ of the surface $\bar{A}$ are subjected to distributed loads which are perpendicular to the surface:

$$
\widetilde{p}_{\mathrm{I}}=p_{\mathrm{I}} \tilde{p}_{\mathrm{I} 0}, \quad \overline{\mathbf{r}} \in \bar{A}_{\mathrm{tI}} ; \quad \widetilde{p}_{\mathrm{II}}=p_{\mathrm{II}} \tilde{p}_{\mathrm{II} 0}, \quad \overline{\mathbf{r}} \in \bar{A}_{\mathrm{tII}} .
$$

Here $p_{\mathrm{I}}$ are $p_{\mathrm{II}}$ independent load parameters whereas $\widetilde{p}_{\mathrm{I} 0}$ and $\widetilde{p}_{\mathrm{II} 0}$ are reference loads. The corresponding elementary forces are given by

$$
\mathrm{d} \overline{\mathbf{F}}_{\mathrm{N}}=\widetilde{p}_{\mathrm{N}} \mathrm{d} \overline{\mathbf{A}}, \quad \mathrm{d} \bar{F}_{\mathrm{N} k}=p_{\mathrm{N}} \widetilde{p}_{\mathrm{N} 0} \mathrm{~d} \bar{A}_{k}=p_{\mathrm{N}} \widetilde{p}_{\mathrm{N} 0} Q_{k}{ }^{p} \mathrm{~d} A_{p}, \quad \mathrm{~N}=\mathrm{I}, \mathrm{II}
$$

where $\mathrm{d} \bar{F}_{\mathrm{N} k}$ stands for the components of the elementary $\mathrm{d} \overline{\mathbf{F}}_{\mathrm{N}}$ in configuration $(B)$.

The loaded surface parts $\bar{A}_{\mathrm{tI}}$ and $\bar{A}_{\mathrm{tII}}$ may depend on the geometrical imperfection parameters.

\section{Fundamental equations}

3.1. Introductory remarks. With regard to all that has been said in Section 2 we may conclude that the state of the elastic body for given stiffness tensor $C^{k l p q}$, reference loads $\widetilde{p}_{\text {IO }}, \widetilde{p}_{\text {IIO }}$ and displacement boundary conditions is determined by the displacement field $u_{k}\left(x^{1}, x^{2}, x^{3}\right)$, the loading parameters $p_{\mathrm{I}}, p_{\mathrm{II}}$ and the geometrical imperfection parameter $h . p_{\mathrm{I}}$ is the parameter of the fundamental load, whereas $p_{\text {II }}$ is the parameter of the supplementary load which depends on what nature the problem considered has, i.e., it may also be the parameter of the disturbing load (load imperfection parameter). $p_{\mathrm{I}}, p_{\mathrm{II}}$ and $h$ are referred to as problem parameters. The imperfection parameters fall into the following two categories: load imperfection parameter $p_{\text {II }}$ and geometrical imperfection parameter $h$. The displacement field $u_{k}$ depends naturally on the problem parameters and can, therefore, be expressed in the form $u_{k}\left(x^{1}, x^{2}, x^{3} ; p_{\mathrm{I}}, p_{\mathrm{II}}, h\right)$

\subsection{Equilibrium conditions.}

3.2.1. Principles of virtual power and virtual work. The current configuration $(\bar{B})$ is an equilibrium state of the body under given $p_{I}, p_{I I}$ and $h$ problem parameters if the principle of virtual power

$$
\int_{(B)} \delta \dot{E}_{k l} C^{k l p q} E_{p q} \mathrm{~d} V=\sum_{\mathrm{N}=\mathrm{I}}^{\mathrm{II}} p_{\mathrm{N}} \int_{\left(A_{\mathrm{tN}}\right)} \widetilde{p}_{\mathrm{N} 0} \delta \dot{u}^{k} Q_{k}^{p} \mathrm{~d} A_{p}
$$

regarded in the initial configuration holds for any virtual velocity field $\delta \dot{u}^{k}$. 
Configuration $(\bar{B})$ is also an equilibrium state of the body if, equivalently to $(3.1)$, the principle of virtual work

$$
\int_{(B)} \delta \Delta E_{k l}^{(1)} C^{k l p q} E_{p q} \mathrm{~d} V=\sum_{\mathrm{N}=\mathrm{I}}^{\mathrm{II}} p_{\mathrm{N}} \int_{\left(A_{\mathrm{tN}}\right)} \widetilde{p}_{\mathrm{N} 0} \delta u^{k} Q_{k}^{p} \mathrm{~d} A_{p}
$$

is fulfilled for any virtual displacement field $\delta u^{k}$.

According to equations (2.3), (2.6), (2.7) and $(2.1)_{3}$

$$
\begin{aligned}
\delta \dot{E}_{k l} C^{k l p q} & =\delta \dot{u}_{m ; k}\left(\delta_{l}^{m}+u_{; l}^{m}\right) C^{k l p q}, \\
\delta \Delta E_{k l}^{(1)} C^{k l p q} & =\delta u_{m ; k}\left(\delta_{l}^{m}+u_{; l}^{m}\right) C^{k l p q}, \quad \delta \Delta E_{k l}^{(2)} C^{k l p q}=\delta u_{m ; k} \Delta u_{; l}^{m} C^{k l p q} .
\end{aligned}
$$

A comparison of $(3.3)$ and $(3.4)_{1}$ shows that $(3.1)$ and $(3.2)$ are really equivalent to each other.

3.2.2. The Newton-Raphson iteration. The equilibrium conditions of the current configuration $(\bar{B})$, which is determined by the problem parameters $p_{\mathrm{I}}, p_{\mathrm{II}}, h$, can also be expressed with respect to an arbitrary but appropriately chosen initial state $\left(B_{1}^{\prime}\right)$ of the body by using the principle of virtual work. Configuration $\left(B_{1}^{\prime}\right)$ is not necessarily equilibrated and the description is again Lagrangian, which means that the principle of virtual work is regarded in the state $B$ of the body.

If $u_{\underline{1} k}$ is the displacement field of the configuration $\left(B_{\underline{1}}^{\prime}\right)$ the displacement field of state $(\bar{B})$ of the body is of the form $u_{k}=u_{\underline{1} k}+\Delta u_{k}$ and the virtual work principle can be given as

$$
\int_{(B)} \delta \Delta E_{k l} C^{k l p q}\left(E_{p q}+\Delta E_{p q}\right) \mathrm{d} V=\sum_{\mathrm{N}=\mathrm{I}}^{\mathrm{II}} p_{\mathrm{N}} \int_{\left(A_{\mathrm{tN}}\right)} \widetilde{p}_{\mathrm{N} 0} \delta u^{k}\left(Q_{k}^{p}+\Delta Q_{k}{ }^{p}\right) \mathrm{d} A_{p} .
$$

or by detailing the increments as

$$
\begin{aligned}
\int_{(B)}\left(\delta \Delta E_{k l}^{(1)}+\delta \Delta E_{k l}^{(2)}\right) C^{k l p q}\left(E_{p q}+\Delta E_{p q}^{(1)}+\Delta E_{p q}^{(2)}\right) \mathrm{d} V= \\
=\sum_{\mathrm{N}=\mathrm{I}} p_{\mathrm{N}} \int_{\left(A_{\mathrm{tN}}\right)} \widetilde{p}_{\mathrm{N} 0} \delta u^{k}\left(Q_{k}{ }^{p}+\Delta Q_{k}^{(1)}{ }_{k}^{p}+\Delta Q_{k}^{(2) p}\right) \mathrm{d} A_{p} .
\end{aligned}
$$

Condition 3.6 is a non-linear equation for the displacement increment $\Delta u_{k}$ as the unknown. The solution can be found by applying the Newton-Raphson iteration algorithm. According to this the displacement field $u_{k}$ of the configuration $(\bar{B})$ can be approximated by the displacement increment series

$$
u_{k}+\Delta u_{k}=u_{\underline{1} k}+\Delta u_{\underline{1} k}+\Delta u_{\underline{2} k}+\cdots+\Delta u_{\underline{s} k}+\cdots
$$

in which $\Delta u_{\underline{\mathrm{s}} k}, \quad(\underline{\mathrm{s}}=\underline{1}, \underline{2}, \cdots)$ is the displacement increment that belongs to the $\underline{s}^{\text {th }}$ intermediate state of the body in the series $\left(B_{\underline{1}}^{\prime}\right),\left(B_{\underline{2}}^{\prime}\right), \cdots,\left(B_{\underline{s}}^{\prime}\right), \cdots$. 
By dropping the terms

$$
\begin{gathered}
\int_{(B)}\left(\delta \Delta E_{\underline{\mathrm{s}} k l}^{(2)} C^{k l p q} \Delta E_{\underline{\mathrm{s}} p q}^{(1)}+\delta \Delta E_{\underline{\mathrm{s}} k l}^{(2)} C^{k l p q} \Delta E_{\underline{\mathrm{s} p q}}^{(2)}\right) \mathrm{d} V, \\
\sum_{\mathrm{N}=\mathrm{I}}^{\mathrm{II}} p_{\mathrm{N}} \int_{\left(A_{\mathrm{tN}}\right)} \widetilde{p}_{\mathrm{N} 0} \delta u^{k} \Delta Q_{\underline{\underline{s}} k}^{(2)} p \mathrm{~d} A_{p}
\end{gathered}
$$

non-linear in (3.6) one can obtain a linearized equation for the unknown $\Delta u_{\underline{s} k}$ :

$$
\begin{aligned}
\int_{(B)}\left(\delta \Delta E_{\underline{\mathrm{s}} k l}^{(1)} C^{k l p q} \Delta E_{\underline{\mathrm{s}} p q}^{(1)}+\delta \Delta E_{\underline{\mathrm{s}} k l}^{(2)} C^{k l p q} E_{\underline{\mathrm{s}} p q}\right) \mathrm{d} V- & \\
-\sum_{\mathrm{N}=\mathrm{I}}^{\mathrm{II}} p_{\mathrm{N}} \int_{\left(A_{\mathrm{tN}}\right)} \widetilde{p}_{\mathrm{N} 0} \delta u^{k} \Delta Q_{\underline{s} k}^{(1) p} \mathrm{~d} A_{p} & =-\int_{(B)} \delta \Delta E_{\underline{\mathrm{s}} k l}^{(1)} C^{k l p q} E_{\underline{\mathrm{s}} p q} \mathrm{~d} V+ \\
& +\sum_{\mathrm{N}=\mathrm{I}}^{\mathrm{II}} p_{\mathrm{N}} \int_{\left(A_{\mathrm{tN}}\right)} \widetilde{p}_{\mathrm{N} 0} \delta u^{k} Q_{\underline{\mathrm{s}} k} p \mathrm{~d} A_{p} .
\end{aligned}
$$

The solution for $u_{\underline{s} k}$ is given by

$$
u_{\underline{\mathrm{s}} k}=u_{\underline{1} k}+\Delta u_{\underline{1} k}+\Delta u_{\underline{2} k}+\cdots+\Delta u_{\underline{\mathrm{s}-1} k} ; \quad \Delta u_{\underline{\mathrm{s}} k} .
$$

The prescribed error limits should be satisfied at the end of the iteration. Then $\Delta u_{\underline{s} k} \rightarrow 0, u_{\underline{s} k} \rightarrow u_{k}, E_{\underline{\mathrm{s}} p q} \rightarrow E_{p q}$, hence the left side of (3.7) tends to zero and the right side coincides with equation $(3.2)$, which expresses the equilibrium of the state $(\bar{B})$.

The $\underline{s}^{\text {th }}$ step of the Newton-Raphson iteration algorithm can be given in a finite element formulation as well:

$$
\mathbf{K}_{\underline{\mathrm{s}} i j} \Delta \mathbf{t}_{\underline{\mathrm{s}} j}=-\mathbf{b}_{\underline{\mathrm{s}} j}+\sum_{\mathrm{N}=\mathrm{I}}^{\mathrm{II}} p_{\mathrm{N}} \mathbf{g}_{\underline{\mathrm{N}} \mathrm{N}}
$$

where by omitting the subscript $\underline{\mathrm{s}}$ that identifies the $\underline{s}^{\text {th }}$ step

$$
\mathbf{K}_{i j}=\mathbf{K}_{i j}^{\mathrm{L}}+\mathbf{K}_{i j}^{\mathrm{G}}-\sum_{\mathrm{N}=\mathrm{I}}^{\mathrm{II}} p_{\mathrm{N}} \mathbf{K}_{\mathrm{N} i j}^{\mathrm{C}}, \quad \mathbf{K}_{i j}^{\mathrm{L}}+\mathbf{K}_{i j}^{\mathrm{G}}=\mathbf{K}_{i j}^{\mathrm{T}} .
$$

Here utilizing the usual technical terms

$\mathbf{K}_{i j}$ is the total stiffness matrix,

$\mathbf{K}_{i j}^{\mathrm{L}}$ is the linear stiffness matrix,

$\mathbf{K}_{i j}^{\mathrm{G}}$ is the geometric stiffness matrix,

$\mathbf{K}_{\mathrm{N} i j}^{\mathrm{C}}$ denotes the load correction stiffness matrices $(N=I, I I)$,

$\mathbf{K}_{i j}^{\mathrm{T}}$ is the tangent stiffness matrix,

$\mathbf{t}_{j}$ is the generalized node displacement matrix.

Equations of the 3D continuum mechanical model and those of the finite element model are related with each other via the following relations 


$$
\begin{gathered}
\int_{(B)} \delta \Delta E_{k l}^{(1)} C^{k l p q} \Delta E_{p q}^{(1)} \mathrm{d} V=\int_{(B)} \delta u_{m ; k}\left(\delta_{l}^{m}+u_{; l}^{m}\right) C^{k l p q}\left(\delta_{p}^{s}+u_{; l}^{s}\right) \Delta u_{s ; q} \mathrm{~d} V= \\
=\delta \mathbf{t}_{i}^{\mathrm{T}} \mathbf{K}_{i j}^{\mathrm{L}} \Delta \mathbf{t}_{j}, \\
\int_{(B)} \delta \Delta E_{k l}^{(2)} C^{k l p q} E_{p q} \mathrm{~d} V=\int_{(B)} \delta u_{m ; k} \Delta u_{; l}^{m} C^{k l p q} E_{p q} \mathrm{~d} V=\delta \mathbf{t}_{i}^{\mathrm{T}} \mathbf{K}_{i j}^{\mathrm{G}} \Delta \mathbf{t}_{j}, \\
\int_{\left(A_{\mathrm{tN}}\right)} \widetilde{p}_{\mathrm{N} 0} \delta u^{k} \Delta Q_{\underline{\mathrm{s}} k}^{(1)}{ }^{p} \mathrm{~d} A_{p}=\int_{\left(A_{\mathrm{tN})}\right.} \widetilde{p}_{\mathrm{N} 0} \delta u^{k} e_{k l m} e^{p q r}\left(\delta_{q}^{l}+u_{; q}^{l}\right) \Delta u_{; r}^{m} \mathrm{~d} A_{p}= \\
\int_{(B)} \delta \Delta E_{k l}^{(1)} C^{k l p q} E_{p q} \mathrm{~d} V=\int_{(B)} \delta \mathbf{t}_{i}^{\mathrm{T}} \mathbf{K}_{\mathrm{N} i j}^{\mathrm{C}} \Delta \mathbf{t}_{j} ; \quad \mathrm{N}=\mathrm{I}, \mathrm{II}, \\
\left.\int_{\left(A_{\mathrm{tN}}\right)} \widetilde{p}_{\mathrm{N} 0} \delta u^{k} Q_{k}^{p} \mathrm{~d} A_{p}=\delta \mathbf{t}_{i}^{\mathrm{T}} \mathbf{g}_{\mathrm{N} i} ; \quad u_{; l}^{m}\right) C^{k l p q} E_{p q} \mathrm{~d} V=\delta \mathbf{t}_{i}^{\mathrm{T}} \mathbf{b}_{i},
\end{gathered}
$$

The load vectors $\mathbf{b}_{i}$ and $\mathbf{g}_{\mathrm{N} i}$ are defined by equations 3.14 and 3.15 .

REMARK 3.1: Here the conventional FEM is enlarged by the introduction of the loadcorrection stiffness matrices in (3.10). It is, however, worth mentioning that the iteration algorithm (3.7)- (3.10) also works if the load correction matrices are taken with a slight modification from the right side of the equation to the left side. If this is the case the number of iteration steps needed to achieve a given error limit is, however, significantly increased according to the numerical experiments.

REMARK 3.2: Let the non-equilibrium state $\left(\bar{B}_{1}^{\prime}\right)$ be a starting point. Then the NewtonRaphson iteration algorithm based on equation 3.6 results in the equilibrium state $(\bar{B})$ under the loading parameters $p_{\mathrm{I}}, p_{\mathrm{II}}$. This procedure can also be applied in the same manner for determining the equilibrium state $\bar{B}+\Delta \bar{B}$ under the loading parameters $p_{\mathrm{I}}+\Delta p_{\mathrm{I}}, p_{\mathrm{II}}+\Delta p_{\mathrm{II}}$ by regarding the earlier equilibrium state $\bar{B}$ as the point of departure.

REMARK 3.3: The problem parameters are constant quantities in the previous part of the present section.

\subsubsection{Equilibrium paths, equilibrium surfaces and critical equilibrium states.}

3.2.3.1. By an equilibrium path/surface is meant such a curve/surface which depicts the displacement $t_{m}$ as a function of $p_{\mathrm{I}} / p_{\mathrm{I}}$ and $p_{\mathrm{II}}$ (or $h$ ) in the interval of the investigated problem parameters. Here and in the sequel the subscript $m$ is fixed when the matrix notation is used, i.e., it does not take any other value.

An equilibrium path is obtained in the coordinate system $\left(p_{\mathrm{I}}, t_{m}\right)$ if $h=h_{0}=$ constant and $p_{\mathrm{II}}=p_{\mathrm{II} 0}=$ constant.

An equilibrium surface is obtained in the coordinate system $\left(p_{\mathrm{II}}, p_{\mathrm{I}}, t_{m}\right)$ if $h=$ $h_{0}=$ constant. The intersections of the equilibrium surface with the coordinate planes $p_{\mathrm{II}}=$ constant or $p_{\mathrm{I}}=$ constant results in a series of equilibrium paths.

The surface $p_{\mathrm{II}}=p_{\mathrm{II} 0}=$ constant is also an equilibrium surface in the coordinate system $\left(h, p_{\mathrm{I}}, t_{m}\right)$. Then the intersections with the coordinate planes $h=$ constant or $p_{\mathrm{I}}=$ constant or $t_{m}=$ constant) yield again a series of equilibrium paths. 
If we proceed we can conclude that a system of surfaces is obtained if the parameter $h$ is changed in the coordinate system $\left(p_{\mathrm{II}}, p_{\mathrm{I}}, t_{m}\right)$. In the same way a system of surfaces is obtained again if the parameter $p_{\text {II }}$ is changed in the coordinate system $\left(h, p_{\mathrm{I}}, t_{m}\right)$.

In order to make the introduced terminology and concepts clearer some special cases will be defined in the sequel. It should, however, be remarked that the possibilities for the problem parameters are considered only partly.

3.2.3.2. First the case $h=0$ is considered. Assuming that the problem has one load parameter only, i.e., $p_{\text {II }}=0$, the following terminology has come into general use for many problems: $p_{\mathrm{I}}$ as loading parameter, fundamental equilibrium path (or primary equilibrium path), which starts from the point $p_{\mathrm{I}}=0$ that corresponds to the stress and deformation free state $(B)$ of the body, and secondary equilibrium path (in some cases it is called the bifurcational equilibrium path) which characterizes the postcritical states. Then $p_{\mathrm{I}} \geq p_{I}^{\text {crit }}$ where $p_{I}^{\text {crit }}$ is the critical value of the one parameter load.

A point on the secondary equilibrium path can be obtained by determining the equilibrium state $(\bar{B})$ if the state $\left(B_{1}^{\prime}\right)$ which belongs to the loading parameter $p_{\mathrm{I}}>$ $p_{I}^{\text {crit }}$ is the starting point for the Newton-Raphson iteration.

From here other points can be calculated on the secondary equilibrium path if the loading parameter is set to $p_{\mathrm{I}}+\Delta p_{\mathrm{I}}$.

The Newton-Raphson iteration can be parametrized. It is possible to select the load $p_{\mathrm{I}}$ as a parameter (loading parametrization) or a displacement component (for instance $t_{m}$ ) as parameter (displacement parametrization).

In this sense the common point of the primary path and the secondary path is a branching point (bifurcation point) which determines the value $p_{\mathrm{I}}=p_{I}^{\text {crit }}$ of the load (path following method).

There is a further method for finding the critical load. This consists in determining the loading parameter that belongs to the smallest non-zero eigenvalue of the corresponding eigenvalue problem (determinant observing method):

$$
\mathbf{K}_{i j} \Delta \mathbf{t}_{j}=\mathbf{0}, \quad \Delta \mathbf{t}_{j} \neq \mathbf{0}, \quad \operatorname{det} \mathbf{K}_{i j}=0 .
$$

The equilibrium paths can also be sought one by one for the values $p_{\mathrm{II}}=$ constant $\neq$ 0 as well.

In all that has been said above the role of the parameters $p_{\mathrm{I}}$ and $p_{\mathrm{II}}$ is interchangeable, i.e., it is possible to determine equilibrium paths if $p_{\mathrm{I}}=$ constant $\neq 0$. Then $p_{\mathrm{II}}$ is the loading parameter.

For problems with two loading parameters the equilibrium states can be illustrated by equilibrium surfaces. In these cases the loading parameters $p_{\mathrm{I}}, p_{\mathrm{II}}$ and the displacement $t_{m}$ are the variables. The equilibrium paths that belong to the values $p_{\text {I }}=$ constant and $p_{\text {II }}=$ constant are intersections of the equilibrium surface and the planes $p_{\mathrm{I}}=$ constant and $p_{\mathrm{II}}=$ constant. Here $p_{I I}$ can be either a supplementary load or a load imperfection parameter. The equilibrium paths $p_{\mathrm{I}}=$ constant or $p_{\mathrm{II}}=$ constant may have maxima, minima and in some special cases points with vertical tangents. The point with a horizontal tangent, i.e., where the equilibrium path 
has, therefore, a maximum (or minimum), is called the limit point. The intersection point of the primary and secondary equilibrium paths (the primary branching point) and the limit points are called critical points, while the loads that belong to these points are the critical loads. The states of the body under these loads are referred to as critical states. A more rigorous definition for the critical state of the body is given in Subsection 4.1 .

3.2.3.3. By changing the parameter $h$ one can determine the different equilibrium paths, equilibrium surfaces, critical points and critical loads for each distinct value of $h=$ constant.

3.2.3.4. When investigating the effect of (load imperfection for which the parameter is $p_{\text {II }}$ ) [geometrical imperfection for which the parameter is $h$ ] the locus of (limit points) [branching points] is called the curve of (limit points or fold line) [branching points].

3.2.4. Asymptotic numerical method. Nonlinear equilibrium problems can be solved either by using the Newton-Rapshon iteration or some other method: the asymptotic numerical method, for instance. The later method is based on the idea that the characteristic variables of the problem can be expended into a Taylor series in a small neighborhood of the reference state $\bar{B}$ by selecting a scalar for the governing parameter of the problem. This scalar is regarded formally as if it were time. The approximations would consist of a few terms only, though they become more accurate if the number of terms is increased.

The governing parameter can be (a) displacement parameter: $\tau=t_{m}-t_{m 0}$; (b) loading parameter: $\tau=p_{\mathrm{N}}-p_{\mathrm{N} 0}, \mathrm{~N}=\mathrm{I}, \mathrm{II}, ;$ (c) geometric imperfection parameter $\tau=h-h_{0}$; or something else (d) the arc length of the equilibrium path for instance: $\tau=s-s_{0}$ where $t_{m 0}, p_{\mathrm{N} 0}, h_{0}, s_{0}$ belong to the equilibrium state $(\bar{B})$.

For the equilibrium state $(\bar{B})$ it holds that $\tau=0$, whereas $\tau=\Delta \tau$ in the state $(\bar{B}+\Delta \bar{B})$ of the body. For the intermediate states, however, $0<\tau<\Delta \tau$.

If the subscript 0 , which identifies state $(\bar{B})$ of the body, is omitted the Taylor series have the following forms:

$$
\begin{aligned}
u^{k}(\tau) & =u^{k}+\Delta u^{k}, & \Delta u^{k} & =\dot{u}^{k} \tau+\frac{1}{2} \ddot{u}^{k} \tau^{2}+\frac{1}{6} \dddot{u}^{k} \tau^{3}+\cdots, \\
\mathbf{t}_{i}(\tau) & =\mathbf{t}_{i}+\Delta \mathbf{t}_{i}, & \Delta \mathbf{t}_{i} & =\dot{\mathbf{t}}_{i} \tau+\frac{1}{2} \ddot{\mathbf{t}}_{i} \tau^{2}+\frac{1}{6} \dddot{\mathbf{t}}_{i} \tau^{3}+\cdots, \\
E_{k l}(\tau) & =E_{k l}+\Delta E_{k l}, & \Delta E_{k l} & =\dot{E}_{k l} \tau+\frac{1}{2} \ddot{E}_{k l} \tau^{2}+\frac{1}{6} \dddot{E}_{k l} \tau^{3}+\cdots, \\
p_{\mathrm{N}}(\tau) & =p_{\mathrm{N}}+\Delta p_{\mathrm{N}}, & \Delta p_{\mathrm{N}} & =\dot{p}_{\mathrm{N}} \tau+\frac{1}{2} \ddot{p}_{\mathrm{N}} \tau^{2}+\frac{1}{6} \dddot{p}_{\mathrm{N}} \tau^{3} \cdots \quad \mathrm{N}=\mathrm{I}, \mathrm{II}, \\
Q_{k}{ }^{p}(\tau) & =Q_{k}{ }^{p}+\Delta Q_{k}{ }^{p}, & \Delta Q_{k}{ }^{p} & =\dot{Q}_{k}{ }^{p} \tau+\frac{1}{2} \ddot{Q}_{k}{ }^{p} \tau^{2}+\frac{1}{6} \dddot{Q}_{k}^{p} \tau^{3}+\cdots, \\
h(\tau) & =h+\Delta h, & \Delta h & =\dot{h} \tau+\frac{1}{2} \ddot{h}^{2}+\frac{1}{6} \dddot{h} \tau^{3}+\cdots .
\end{aligned}
$$

where the derivatives of $u^{k}, \mathbf{t}_{i}, E_{k l}, p_{\mathrm{N}}, Q_{k}{ }^{p}$ and $h$ with respect to $\tau$ belong to the state $(\bar{B})$. 
The essence of the numerical method consists in determining the unknown derivatives in the state $(\bar{B})$ of the body [see equations (3.41) and (3.42) presented later] and then the increments are calculated by utilizing the above relations and taking the prescribed error limits also into account.

For preparing the derivation of the equilibrium equations we determine the first three derivatives of $E_{k l}$ and $Q_{k}{ }^{p}$ assuming that $h=$ constant:

$$
\begin{gathered}
\dot{E}_{k l} C^{k l p q}=\dot{u}_{m ; k}\left(\delta_{l}^{m}+u_{; l}^{m}\right) C^{k l p q}, \\
\ddot{E}_{k l} C^{k l p q}=\left[\ddot{u}_{m ; k}\left(\delta_{l}^{m}+u_{; l}^{m}\right)+\dot{u}_{m ; k} \dot{u}_{; l}^{m}\right] C^{k l p q}, \\
\dddot{E}_{k l} C^{k l p q}=\left[\dddot{u}_{m ; k}\left(\delta_{l}^{m}+u_{; l}^{m}\right)+2 \ddot{u}_{m ; k} \dot{u}_{; l}^{m}+\dot{u}_{m ; k} \ddot{u}_{; l}^{m}\right] C^{k l p q}, \\
\dot{Q}_{k}^{p}=e_{k l m} e^{p q r}\left(\delta_{q}^{l}+u_{; q}^{l}\right) \dot{u}_{; r}^{m}, \\
\ddot{Q}_{k}^{p}=e_{k l m} e^{p q r}\left[\left(\delta_{q}^{l}+u_{; q}^{l}\right) \ddot{u}_{; r}^{m}+\dot{u}_{; q}^{l} \dot{u}_{; r}^{m}\right], \\
\dddot{Q}_{k}^{p}=e_{k l m} e^{p q r}\left[\left(\delta_{q}^{l}+u_{; q}^{l}\right) \dddot{u}_{; r}^{m}+2 \dot{u}_{; q}^{l} \ddot{u}_{; r}^{m}+\ddot{u}_{; q}^{l} \dot{u}_{; r}^{m}\right] .
\end{gathered}
$$

According to the principle of virtual power state $(\bar{B})(\tau=0)$ is in equilibrium under the problem parameters $p_{\mathrm{I}}, p_{\mathrm{II}}, h$ if 3.1 is fulfilled. With the finite element algorithm we may write

$$
\delta \dot{\mathbf{t}}_{i}^{\mathrm{T}}\left(\mathbf{b}_{i}-\sum_{\mathrm{N}=\mathrm{I}}^{\mathrm{II}} p_{\mathrm{N}} \mathbf{g}_{\mathrm{N} i}\right)=0 \quad \text { from where it follows that } \mathbf{b}_{i}-\sum_{\mathrm{N}=\mathrm{I}}^{\mathrm{II}} p_{\mathrm{N}} \mathbf{g}_{\mathrm{N} i}=\mathbf{0} .
$$

Here in accordance with 3.14 and 3.15

$$
\delta \dot{\mathbf{t}}_{i}^{\mathrm{T}} \mathbf{b}_{i}=\int_{(B)} \delta \dot{E}_{k l} C^{k l p q} E_{p q} \mathrm{~d} V=\int_{(B)} \delta \dot{u}_{m ; k}\left(\delta_{l}^{m}+u_{; l}^{m}\right) C^{k l p q} E_{p q} \mathrm{~d} V
$$

and

$$
\begin{aligned}
\delta \dot{\mathbf{t}}_{i}^{\mathrm{T}} \mathbf{g}_{\mathrm{N} i} & =\int_{\left(A_{\mathrm{tN}}\right)} \widetilde{p}_{\mathrm{N} 0} \delta \dot{u}^{k} Q_{k}^{p} \mathrm{~d} A_{p}= \\
& =\int_{\left(A_{\mathrm{tN}}\right)} \widetilde{p}_{\mathrm{N} 0} \delta \dot{u}^{k} \frac{1}{2} e_{k l m} e^{p q r}\left(\delta_{q}^{l}+u_{; q}^{l}\right)\left(\delta_{r}^{m}+u_{; r}^{m}\right) \mathrm{d} A_{p} ; \quad \mathrm{N}=\mathrm{I}, \mathrm{II} .
\end{aligned}
$$

Introducing the notation

$$
\mathbf{A}_{i}=\mathbf{b}_{i}-\sum_{\mathrm{N}=\mathrm{I}}^{\mathrm{II}} p_{\mathrm{N}} \mathbf{g}_{\mathrm{N} i}
$$

equation 3.19 can be rewritten into the following form:

$$
\delta \dot{\mathbf{t}}_{i}^{\mathrm{T}} \mathbf{A}_{i}=0 \quad \text { which means that } \quad \mathbf{A}_{i}=\mathbf{0} .
$$

The virtual variables $\delta \dot{u}^{k}$ and $\delta \dot{\mathbf{t}}_{i}$ are independent of the governing parameter $\tau$. Hence

$$
\left(\delta \dot{u}^{k}\right)^{\cdot}=\left(\delta \dot{u}^{k}\right)^{*}=\cdots=0, \quad\left(\delta \dot{\mathbf{t}}_{i}\right)^{\cdot}=\left(\delta \dot{\mathbf{t}}_{i}\right)^{*}=\cdots=0 .
$$

For the general case it is assumed that the geometric imperfection parameter depends also on the governing parameter $\tau$. The following relations are, therefore, assumed 
for the general case:

$$
\begin{array}{rcc}
u^{k}(\tau), & \mathbf{t}_{i}(\tau), \quad p_{\mathrm{N}}(\tau), & h(\tau), \\
\mathbf{b}_{i}\left(\mathbf{t}_{j}, h\right), & \mathbf{g}_{\mathrm{N} i}\left(\mathbf{t}_{j}, h\right), & \mathbf{A}_{i}\left(\mathbf{t}_{j}, p_{\mathrm{N}}, h\right) .
\end{array}
$$

Equations 3.17a -3.19 are also valid for the general case. Some special cases are given below:

$\checkmark \tau=p_{\mathrm{I}}-p_{\mathrm{I} 0} ; \quad \dot{p}_{\mathrm{I}}=1, \ddot{p}_{\mathrm{I}}, \dddot{p}_{\mathrm{I}}, \cdots=0 ; \quad \dot{p}_{\mathrm{II}}, \ddot{p}_{\mathrm{II}}, \cdots=0 ; \quad h=h_{0}, \dot{h}, \ddot{h}, \cdots=0$,

$\checkmark=p_{\text {II }}-p_{\text {II } 0} ; \quad \dot{p}_{\text {II }}=1, \quad \ddot{p}_{\text {II }}, \dddot{p}_{\text {II }}, \cdots=0 ; \quad \dot{p}_{\mathrm{I}}, \ddot{p}_{\mathrm{I}}, \cdots=0 ; \quad h=h_{0}, \dot{h}, \ddot{h}, \cdots=0$,

$\checkmark \tau=h-h_{0} ; \quad \dot{h}=1, \ddot{h}, \dddot{h}, \cdots=0 ; \quad \dot{p}_{\mathrm{I}}, \ddot{p}_{\mathrm{I}}, \cdots=0 ; \quad \ddot{p}_{\mathrm{II}}, \dddot{p}_{\mathrm{II}}, \cdots=0$.

Let us expend the equilibrium equation into series in terms of the governing parameter $\tau$ in a small neighborhood of the equilibrium state $(\bar{B})$ by taking equations 3.24 and relationships 3.25 into account. We have

$$
\delta \dot{\mathbf{t}}_{i}^{\mathrm{T}} \mathbf{A}_{i}+\left(\delta \dot{\mathbf{t}}_{i}^{\mathrm{T}} \mathbf{A}_{i}\right)^{\cdot} \tau+\frac{1}{2}\left(\delta \dot{\mathbf{t}}_{i}^{\mathrm{T}} \mathbf{A}_{i}\right)^{\cdot} \tau^{2}+\frac{1}{6}\left(\delta \dot{\mathbf{t}}_{i}^{\mathrm{T}} \mathbf{A}_{i}\right)^{\cdots} \tau^{3}+\cdots=0,
$$

from where it follows with regard to 3.23$)_{2}$ that:

$$
\begin{aligned}
\delta \dot{\mathbf{t}}_{i}^{\mathrm{T}}\left(\frac{\partial \mathbf{A}_{i}}{\partial \mathbf{t}_{j}} \dot{\mathbf{t}}_{j}+\sum_{\mathrm{N}=\mathrm{I}}^{\mathrm{II}} \frac{\partial \mathbf{A}_{i}}{\partial p_{\mathrm{N}}} \dot{p}_{\mathrm{N}}+\frac{\partial \mathbf{A}_{i}}{\partial h} \dot{h}\right) \tau+ \\
+\frac{1}{2} \delta \dot{\mathbf{t}}_{i}^{\mathrm{T}}\left(\frac{\partial \mathbf{A}_{i}}{\partial \mathbf{t}_{j}} \ddot{\mathbf{t}}_{j}+\sum_{\mathrm{N}=\mathrm{I}}^{\mathrm{II}} \frac{\partial \mathbf{A}_{i}}{\partial p_{\mathrm{N}}} \ddot{p}_{\mathrm{N}}+\frac{\partial \mathbf{A}_{i}}{\partial h} \ddot{h}\right) \tau^{2}+ \\
+\frac{1}{2} \delta \dot{\mathbf{t}}_{i}^{\mathrm{T}}\left(\frac{\partial}{\partial \mathbf{t}_{k}} \frac{\partial \mathbf{A}_{i}}{\partial \mathbf{t}_{j}} \dot{\mathbf{t}}_{k} \dot{\mathbf{t}}_{j}+\sum_{\mathrm{N}=\mathrm{I}}^{\mathrm{II}} \frac{\partial^{2} \mathbf{A}_{i}}{\partial p_{\mathrm{N}}^{2}} \dot{p}_{\mathrm{N}}^{2}+\frac{\partial^{2} \mathbf{A}_{i}}{\partial h^{2}} \dot{h}^{2}\right) \tau^{2}+ \\
+\frac{1}{2} \delta \dot{\mathbf{t}}_{i}^{\mathrm{T}}\left(\sum_{\mathrm{N}=\mathrm{I}}^{\mathrm{II}} 2 \frac{\partial}{\partial p_{\mathrm{N}}} \frac{\partial \mathbf{A}_{i}}{\partial \mathbf{t}_{j}} \dot{\mathbf{t}}_{j} \dot{p}_{\mathrm{N}}+2 \frac{\partial}{\partial h} \frac{\partial \mathbf{A}_{i}}{\partial \mathbf{t}_{j}} \dot{\mathbf{t}}_{j} \dot{h}+\sum_{\mathrm{N}=\mathrm{I}}^{\mathrm{II}} 2 \frac{\partial}{\partial h} \frac{\partial \mathbf{A}_{i}}{\partial p_{\mathrm{N}}} \dot{p}_{\mathrm{N}} \dot{h}\right) \tau^{2}+\cdots=0 .
\end{aligned}
$$

REMARK 3.4: The terms in the power series for which the power of $\tau$ is higher than two contain the derivatives $\frac{\partial}{\partial \mathbf{t}_{m}} \frac{\partial}{\partial \mathbf{t}_{k}} \frac{\partial \mathbf{A}_{i}}{\partial \mathbf{t}_{j}}$ of the matrices $\mathbf{A}_{i}$ only. The reason for this is simple: according to equations (3.20) and (2.2) $\mathbf{A}_{i}$ does not contain the power of $u^{m}$ and its derivatives higher than three.

Making use of equations 3.22, 3.20 and (3.21) we shall give the details for equation 3.26 :

As regards the expression

$$
\delta \dot{\mathbf{t}}_{i}^{\mathrm{T}} \frac{\partial \mathbf{A}_{i}}{\partial \mathbf{t}_{j}} \dot{\mathbf{t}}_{j}=\delta \dot{\mathbf{t}}_{i}^{\mathrm{T}}\left(\frac{\partial \mathbf{b}_{i}}{\partial \mathbf{t}_{j}}-\sum_{\mathrm{N}=\mathrm{I}}^{\mathrm{II}} p_{\mathrm{N}} \frac{\partial \mathbf{g}_{\mathrm{N} i}}{\partial \mathbf{t}_{j}}\right) \dot{\mathbf{t}}_{j},
$$

we get

$$
\delta \dot{\mathbf{t}}_{i}^{\mathrm{T}} \frac{\partial \mathbf{b}_{i}}{\partial \mathbf{t}_{j}} \dot{\mathbf{t}}_{j}=\int_{(B)} \delta \dot{u}_{m ; k}\left[\left(\delta_{l}^{m}+u_{; l}^{m}\right) C^{k l p q} E_{p q}\right] \mathrm{d} V=
$$




$$
\begin{array}{r}
=\int_{(B)} \delta \dot{u}_{m ; k} \dot{u}_{; l}^{m} C^{k l p q} E_{p q} \mathrm{~d} V+\int_{(B)} \delta \dot{u}_{m ; k}\left(\delta_{l}^{m}+u_{; l}^{m}\right) C^{k l p q}\left(\delta_{p}^{s}+u_{; p}^{s}\right) \dot{u}_{s ; q} \mathrm{~d} V= \\
=\delta \dot{\mathbf{t}}_{i}^{\mathrm{T}}\left(\mathbf{K}_{i j}^{\mathrm{G}} \dot{\mathbf{t}}_{j}+\mathbf{K}_{i j}^{\mathrm{L}} \dot{\mathbf{t}}_{j}\right), \quad(3.28)
\end{array}
$$

and

$$
\begin{aligned}
&-\delta \dot{\mathbf{t}}_{i}^{\mathrm{T}} \sum_{\mathrm{N}=\mathrm{I}}^{\mathrm{II}} p_{\mathrm{N}} \frac{\partial \mathbf{g}_{\mathrm{N} i}}{\partial \mathbf{t}_{j}} \dot{\mathbf{t}}_{j}= \\
&=-\sum_{\mathrm{N}=\mathrm{I}}^{\mathrm{II}} p_{\mathrm{N}} \int_{\left(A_{\mathrm{tN}}\right)} \widetilde{p}_{\mathrm{N} 0} \delta \dot{u}^{k} \frac{1}{2} e_{k l m} e^{p q r}\left[\left(\delta_{q}^{l}+u_{; q}^{l}\right)\left(\delta_{r}^{m}+u_{; r}^{m}\right)\right] \mathrm{d} A_{p}= \\
&=-\sum_{\mathrm{N}=\mathrm{I}}^{\mathrm{II}} p_{\mathrm{N}} \int_{\left(A_{\mathrm{tN}}\right)} \widetilde{p}_{\mathrm{N} 0} \delta \dot{u}^{k} e_{k l m} e^{p q r}\left(\delta_{q}^{l}+u_{; q}^{l}\right) \dot{u}_{; r}^{m} \mathrm{~d} A_{p}= \\
&=-\delta \dot{\mathbf{t}}_{i}^{\mathrm{T}} \sum_{\mathrm{N}=\mathrm{I}}^{\mathrm{II}} p_{\mathrm{N}} \mathbf{K}_{\mathrm{N} i j}^{\mathrm{C}} \dot{\mathbf{t}}_{j} .
\end{aligned}
$$

We shall proceeded in the same way by detailing the terms in equation 3.26 one by one:

$$
\begin{aligned}
& \delta \dot{\mathbf{t}}_{i}^{\mathrm{T}} \frac{\partial \mathbf{A}_{i}}{\partial p_{\mathrm{N}}} \dot{p}_{\mathrm{N}}=-\delta \dot{\mathbf{t}}_{i} \sum_{\mathrm{N}=\mathrm{I}}^{\mathrm{II}} \frac{\partial}{\partial p_{\mathrm{N}}}\left(\sum_{\mathrm{M}=\mathrm{I}}^{\mathrm{II}} p_{\mathrm{M}} \mathbf{g}_{\mathrm{M} i}\right) \dot{p}_{\mathrm{N}}=-\delta \dot{\mathbf{t}}_{i}^{\mathrm{T}} \sum_{\mathrm{N}=\mathrm{I}}^{\mathrm{II}} \mathbf{g}_{\mathrm{N} i} \dot{p}_{\mathrm{N}}, \\
& \delta \dot{\mathbf{t}}_{i}^{\mathrm{T}} \frac{\partial \mathbf{A}_{i}}{\partial h} \dot{h}=\delta \mathbf{t t}_{i}^{\mathrm{T}}\left(\frac{\partial \mathbf{b}_{i}}{\partial h}-\sum_{\mathrm{N}=\mathrm{I}}^{\mathrm{II}} p_{\mathrm{N}} \frac{\partial \mathbf{g}_{\mathrm{N} i}}{\partial h}\right) \dot{h}, \\
& \frac{1}{2} \delta \dot{\mathbf{t}}_{i}^{\mathrm{T}}\left(\frac{\partial \mathbf{A}_{i}}{\partial \mathbf{t}_{j}} \ddot{\mathbf{t}}_{j}+\sum_{\mathrm{N}=\mathrm{I}}^{\mathrm{II}} \frac{\partial \mathbf{A}_{i}}{\partial p_{\mathrm{N}}} \ddot{p}_{\mathrm{N}}+\frac{\partial \mathbf{A}_{i}}{\partial h} \ddot{h}\right)= \\
& =\frac{1}{2} \delta \dot{\mathbf{t}}_{i}^{\mathrm{T}}\left[\left(\mathbf{K}_{i j}^{\mathrm{L}}+\mathbf{K}_{i j}^{\mathrm{G}}-\sum_{\mathrm{N}=\mathrm{I}}^{\mathrm{II}} p_{\mathrm{N}} \mathbf{K}_{\mathrm{N} i j}^{\mathrm{C}}\right) \ddot{\mathbf{t}}_{j}-\sum_{\mathrm{N}=\mathrm{I}}^{\mathrm{II}} \mathbf{g}_{\mathrm{N} i} \ddot{p}_{\mathrm{N}}+\right. \\
& \left.+\left(\frac{\partial \mathbf{b}_{i}}{\partial h}-\sum_{\mathrm{N}=\mathrm{I}}^{\mathrm{II}} p_{\mathrm{N}} \frac{\partial \mathbf{g}_{\mathrm{N} i}}{\partial h}\right) \ddot{h}\right], \\
& \frac{1}{2} \delta \dot{\mathbf{t}}_{i}^{\mathrm{T}} \frac{\partial}{\partial \mathbf{t}_{k}} \frac{\partial \mathbf{A}_{i}}{\partial \mathbf{t}_{j}} \dot{\mathbf{t}}_{k} \dot{\mathbf{t}}_{j}=\frac{1}{2} \delta \dot{\mathbf{t}}_{i}^{\mathrm{T}}\left(\frac{\partial}{\partial \mathbf{t}_{k}} \frac{\partial \mathbf{b}_{i}}{\partial \mathbf{t}_{j}} \dot{\mathbf{t}}_{k}-\sum_{\mathrm{N}=\mathrm{I}}^{\mathrm{II}} p_{\mathrm{N}} \frac{\partial}{\partial \mathbf{t}_{k}} \frac{\partial \mathbf{g}_{\mathrm{N} i}}{\partial \mathbf{t}_{j}} \dot{\mathbf{t}}_{k}\right) \dot{\mathbf{t}}_{j}, \\
& \frac{1}{2} \delta \dot{\mathbf{t}}_{i}^{\mathrm{T}} \frac{\partial}{\partial \mathbf{t}_{k}} \frac{\partial \mathbf{b}_{i}}{\partial \mathbf{t}_{j}} \dot{\mathbf{t}}_{k} \dot{\mathbf{t}}_{j}=\frac{1}{2} 2 \int_{(B)} \delta \dot{u}_{m ; k} \dot{u}_{; l}^{m} C^{k l p q}\left(\delta_{p}^{s}+u_{; p}^{s}\right) \dot{u}_{s ; q} \mathrm{~d} V+ \\
& +\frac{1}{2} \int_{(B)} \delta \dot{u}_{m ; k}\left(\delta_{l}^{m}+u_{; l}^{m}\right) C^{k l p q} \dot{u}_{; p}^{s} \dot{u}_{s ; q} \mathrm{~d} V=\frac{1}{2} \delta \dot{\mathbf{t}}_{i}^{\mathrm{T}}\left(2 \mathbf{J}_{i k j}+\mathbf{H}_{i k j}\right) \dot{\mathbf{t}}_{k} \dot{\mathbf{t}}_{j}, \\
& -\frac{1}{2} \delta \dot{\mathbf{t}}_{i}^{\mathrm{T}} \sum_{\mathrm{N}=\mathrm{I}}^{\mathrm{II}} p_{\mathrm{N}} \frac{\partial}{\partial \mathbf{t}_{k}} \frac{\partial \mathbf{g}_{\mathrm{N} i}}{\partial \mathbf{t}_{j}} \dot{\mathbf{t}}_{k} \dot{\mathbf{t}}=
\end{aligned}
$$




$$
\begin{aligned}
& =-\frac{1}{2} \delta \dot{\mathbf{t}}_{i}^{\mathrm{T}} \sum_{\mathrm{N}=\mathrm{I}}^{\mathrm{II}} p_{\mathrm{N}} \int_{\left(A_{\mathrm{tN}}\right)} \widetilde{p}_{\mathrm{N} 0} \delta \dot{u}^{k} e_{k l m} e^{p q r} \dot{u}_{; q}^{l} \dot{u}_{; r}^{m} \mathrm{~d} A_{p}=-\frac{1}{2} \delta \dot{\mathbf{t}}_{i}^{\mathrm{T}} \sum_{\mathrm{N}=\mathrm{I}}^{\mathrm{II}} p_{\mathrm{N}} \mathbf{M}_{\mathrm{N} i k j} \dot{\mathbf{t}}_{k} \dot{\mathbf{t}}_{j}, \\
& \frac{1}{2} \delta \dot{\mathbf{t}}_{i}^{\mathrm{T}} \sum_{\mathrm{N}=\mathrm{I}}^{\mathrm{II}} \frac{\partial^{2} \mathbf{A}_{i}}{\partial p_{\mathrm{N}}^{2}} \dot{p}_{\mathrm{N}}^{2}=0 \\
& \frac{1}{2} \delta \dot{\mathbf{t}}_{i}^{\mathrm{T}} \frac{\partial^{2} \mathbf{A}_{i}}{\partial h^{2}} \dot{h}^{2}=\frac{1}{2} \delta \dot{\mathbf{t}}_{i}^{\mathrm{T}}\left(\frac{\partial^{2} \mathbf{b}_{i}}{\partial h^{2}}-\sum_{\mathrm{N}=\mathrm{I}}^{\mathrm{II}} p_{\mathrm{N}} \frac{\partial^{2} \mathbf{g}_{\mathrm{N} i}}{\partial h^{2}}\right) \dot{h}^{2}, \\
& \frac{1}{2} \delta \dot{\mathbf{t}}_{i}^{\mathrm{T}} \sum_{\mathrm{N}=\mathrm{I}}^{\mathrm{II}} 2 \frac{\partial}{\partial p_{\mathrm{N}}} \frac{\partial \mathbf{A}_{i}}{\partial \mathbf{t}_{j}} \dot{\mathbf{t}}_{j} \dot{p}_{\mathrm{N}}=-\frac{1}{2} \delta \dot{\mathbf{t}}_{i}^{\mathrm{T}} \sum_{\mathrm{N}=\mathrm{I}}^{\mathrm{II}} 2 \frac{\partial}{\partial p_{\mathrm{N}}}\left(\sum_{\mathrm{M}=\mathrm{I}}^{\mathrm{II}} p_{\mathrm{M}} \frac{\partial \mathbf{g}_{\mathrm{M} i}}{\partial \mathbf{t}_{j}} \dot{\mathbf{t}}_{j}\right) \dot{p}_{\mathrm{N}}= \\
& =-\frac{1}{2} \delta \dot{\mathbf{t}}_{i}^{\mathrm{T}}\left(2 \sum_{\mathrm{N}=\mathrm{I}}^{\mathrm{II}} \mathbf{K}_{\mathrm{N} i j}^{\mathrm{C}} \dot{p}_{\mathrm{N}}\right) \dot{\mathbf{t}}_{j}, \\
& \frac{1}{2} \delta \dot{\mathbf{t}}_{i}^{\mathrm{T}} 2 \frac{\partial}{\partial h} \frac{\partial \mathbf{A}_{i}}{\partial \mathbf{t}_{j}} \dot{\mathbf{t}}_{j} \dot{h}=\frac{1}{2} \delta \dot{\mathbf{t}}_{i}^{\mathrm{T}} 2 \frac{\partial}{\partial h}\left(\frac{\partial \mathbf{b}_{i}}{\partial \mathbf{t}_{j}}-\sum_{\mathrm{N}=\mathrm{I}}^{\mathrm{II}} p_{\mathrm{N}} \frac{\partial \mathbf{g}_{\mathrm{N} i}}{\partial \mathbf{t}_{j}}\right) \dot{\mathbf{t}}_{j} \dot{h}= \\
& =\frac{1}{2} \delta \dot{\mathbf{t}}_{i}^{\mathrm{T}} 2 \frac{\partial}{\partial h}\left(\mathbf{K}_{i j}^{\mathrm{L}}+\mathbf{K}_{i j}^{\mathrm{G}}-\sum_{\mathrm{N}=\mathrm{I}}^{\mathrm{II}} p_{\mathrm{N}} \mathbf{K}_{\mathrm{N} i j}^{\mathrm{C}}\right) \dot{\mathbf{t}}_{j} \dot{h}, \\
& \frac{1}{2} \delta \dot{\mathbf{t}}_{i}^{\mathrm{T}} \sum_{\mathrm{N}=\mathrm{I}}^{\mathrm{II}} 2 \frac{\partial}{\partial h} \frac{\partial \mathbf{A}_{i}}{\partial p_{\mathrm{N}}} \dot{p}_{\mathrm{N}} \dot{h}=-\frac{1}{2} \delta \dot{\mathbf{t}}_{i}^{\mathrm{T}} 2\left(\sum_{\mathrm{N}=\mathrm{I}}^{\mathrm{II}} \frac{\partial \mathbf{g}_{\mathrm{N} i}}{\partial h} \dot{p}_{\mathrm{N}}\right) \dot{h} .
\end{aligned}
$$

Since equation (3.26) is fulfilled for any $\tau$ and $\delta \dot{\mathbf{t}}_{i}$ it yields the following equations

$$
\begin{gathered}
\left(\mathbf{K}_{i j}^{\mathrm{L}}+\mathbf{K}_{i j}^{\mathrm{G}}-\sum_{\mathrm{N}=\mathrm{I}}^{\mathrm{II}} \mathbf{K}_{\mathrm{N} i j}^{\mathrm{C}} p_{\mathrm{N}}\right) \dot{\mathbf{t}}_{j}-\sum_{\mathrm{N}=\mathrm{I}}^{\mathrm{II}} \mathbf{g}_{\mathrm{N} i} \dot{p}_{\mathrm{N}}+\left(\frac{\partial \mathbf{b}_{i}}{\partial h}-\sum_{\mathrm{N}=\mathrm{I}}^{\mathrm{II}} \frac{\partial \mathbf{g}_{\mathrm{N} i}}{\partial h} p_{\mathrm{N}}\right) \dot{h}=0, \\
\left(\mathbf{K}_{i j}^{\mathrm{L}}+\mathbf{K}_{i j}^{\mathrm{G}}-\sum_{\mathrm{N}=\mathrm{I}}^{\mathrm{II}} \mathbf{K}_{\mathrm{N} i j}^{\mathrm{C}} p_{\mathrm{N}}\right) \ddot{\mathbf{t}}_{j}-\sum_{\mathrm{N}=\mathrm{I}}^{\mathrm{II}} \mathbf{g}_{\mathrm{N} i} \ddot{p}_{\mathrm{N}}+\left(\frac{\partial \mathbf{b}_{i}}{\partial h}-\sum_{\mathrm{N}=\mathrm{I}}^{\mathrm{II}} \frac{\partial \mathbf{g}_{\mathrm{N} i}}{\partial h} p_{\mathrm{N}}\right) \ddot{h}= \\
=-\left(2 \mathbf{J}_{i k j}+\mathbf{H}_{i k j}-\sum_{\mathrm{N}=\mathrm{I}}^{\mathrm{II}} \mathbf{M}_{\mathrm{N} i k j} p_{\mathrm{N}}\right) \dot{\mathbf{t}}_{k} \dot{\mathbf{t}}_{j}-\left(\frac{\partial^{2} \mathbf{b}_{i}}{\partial h^{2}}-\sum_{\mathrm{N}=\mathrm{I}}^{\mathrm{II}} p_{\mathrm{N}} \frac{\partial^{2} \mathbf{g}_{\mathrm{N} i}}{\partial h^{2}}\right) \dot{h}^{2}+ \\
+2\left(\sum_{\mathrm{N}=\mathrm{I}}^{\mathrm{II}} \mathbf{K}_{\mathrm{N} i j}^{\mathrm{C}} \dot{p}_{\mathrm{N}}\right) \dot{\mathbf{t}}_{j}-2 \frac{\partial}{\partial h}\left(\mathbf{K}_{i j}^{\mathrm{L}}+\mathbf{K}_{i j}^{\mathrm{G}}-\sum_{\mathrm{N}=\mathrm{I}}^{\mathrm{II}} \mathbf{K}_{\mathrm{N} i j}^{\mathrm{C}} p_{\mathrm{N}}\right) \dot{\mathbf{t}}_{j} \dot{h}+ \\
+2\left(\sum_{\mathrm{N}=\mathrm{I}}^{\mathrm{II}} \frac{\partial \mathbf{g}_{\mathrm{N} i}}{\partial h} \dot{p}_{\mathrm{N}}\right) \dot{h} .
\end{gathered}
$$

Equations $(3.19)_{2},(3.41)$ and $(3.42)$ are referred to as equilibrium equations of the asymptotic method. Equation (3.26) can be continued for the higher powers of of $\tau$. Hence, further equilibrium equations can be obtained for the higher derivatives of the problem parameters. 
Two important characteristics of the equation system should be emphasized.

The first is that the coefficient matrices of the time derivatives of the problem parameters on the left side are the same in the successive equations. The second is that the order of the derivatives on the right side is always lower than that of the derivatives on the left side. This makes possible it to calculate the first, second, etc. derivatives of the problem parameters step by step.

If a displacement is the governing parameter the equilibrium equations can be transformed into another form. Let us assume that $\dot{h}=0$ and regard equation (3.41) as an example. Then we can rewrite it into the form

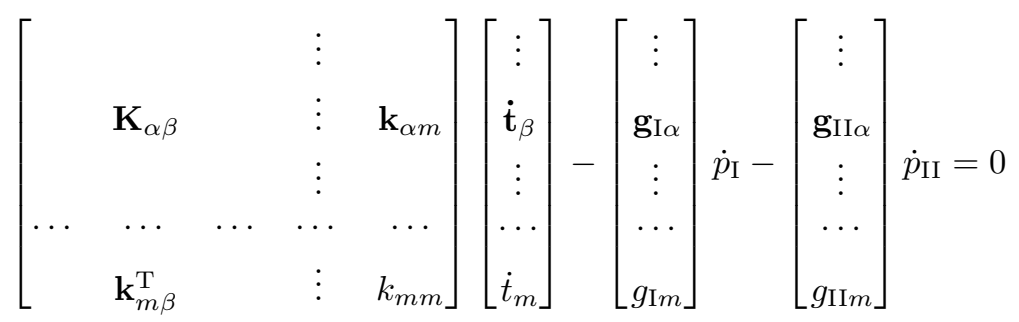

where $m$ is the number of freedom and $\alpha, \beta=1,2, \cdots, m-1$. The corresponding scalar equations are given by

$$
\begin{aligned}
& \mathbf{K}_{\alpha \beta} \dot{\mathbf{t}}_{\beta}+\mathbf{k}_{\alpha m} \dot{t}_{m}-\mathbf{g}_{\mathrm{I} \alpha} \dot{p}_{\mathrm{I}}-\mathbf{g}_{\mathrm{II} \alpha} \dot{p}_{\mathrm{II}}=0, \\
& \mathbf{k}_{m \beta}^{\mathrm{T}} \dot{\mathbf{t}}_{\beta}+k_{m m} \dot{t}_{m}-g_{\mathrm{I} m} \dot{p}_{\mathrm{I}}-g_{\mathrm{II} m} \dot{p}_{\mathrm{II}}=0 .
\end{aligned}
$$

It is clear that these equations can be modified by performing an identity transformation:

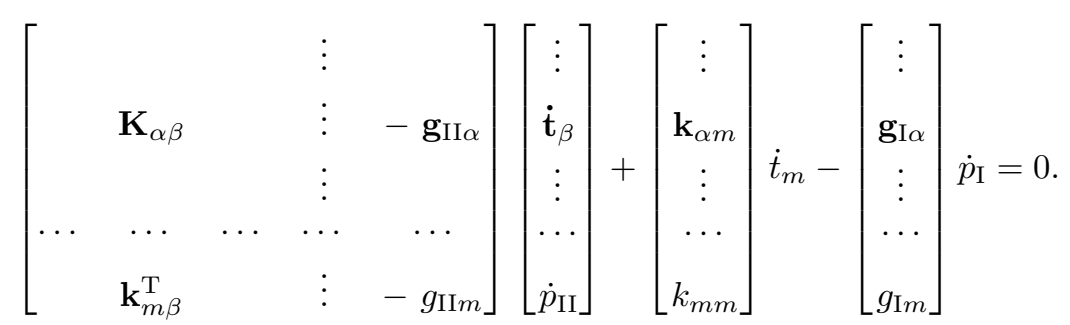

The structure of the equilibrium equations within the framework of the continuum mechanical model can be surveyed in the following manner. Let us consider equation (3.1) as the point of departure:

$$
\int_{(B)} \delta \dot{u}_{m ; k}\left(\delta_{l}^{m}+u_{; l}^{m}\right) C^{k l p q} E_{p q} \mathrm{~d} V-\sum_{\mathrm{N}=\mathrm{I}}^{\mathrm{II}} p_{\mathrm{N}} \int_{\left(A_{\mathrm{tN}}\right)} \widetilde{p}_{\mathrm{N} 0} \delta \dot{u}^{k} Q_{k}^{p} \mathrm{~d} A_{p}=0
$$

from where further equations can be obtained:

$$
\begin{aligned}
\int_{(B)} \delta \dot{u}_{m ; k} & {\left[\left(\delta_{l}^{m}+u_{; l}^{m}\right) C^{k l p q} E_{p q}\right] \mathrm{d} V+} \\
+ & \frac{\partial}{\partial h}\left(\int_{(B)} \delta \dot{u}_{m ; k}\left(\delta_{l}^{m}+u_{; l}^{m}\right) C^{k l p q} E_{p q} \mathrm{~d} V\right) \dot{h}-
\end{aligned}
$$




$$
\begin{aligned}
& -\sum_{\mathrm{N}=\mathrm{I}}^{\mathrm{II}}\left[\dot{p}_{\mathrm{N}} \int_{\left(A_{\mathrm{tN}}\right)} \widetilde{p}_{\mathrm{N} 0} \delta \dot{u}^{k} Q_{k}^{p} \mathrm{~d} A_{p}+p_{\mathrm{N}} \int_{\left(A_{\mathrm{tN}}\right)} \widetilde{p}_{\mathrm{N} 0} \delta \dot{u}^{k} \dot{Q}_{k}^{p} \mathrm{~d} A_{p}+\right. \\
& \left.+p_{\mathrm{N}} \frac{\partial}{\partial h}\left(\int_{\left(A_{\mathrm{tN}}\right)} \widetilde{p}_{\mathrm{N} 0} \delta \dot{u}^{k} Q_{k}^{p} \mathrm{~d} A_{p}\right) \dot{h}\right]=0, \\
& \int_{(B)} \delta \dot{u}_{m ; k}\left[\left(\delta_{l}^{m}+u_{; l}^{m}\right) C^{k l p q} E_{p q}\right]^{\cdot} \mathrm{d} V+ \\
& +2 \frac{\partial}{\partial h}\left(\int_{(B)} \delta \dot{u}_{m ; k}\left[\left(\delta_{l}^{m}+u_{; l}^{m}\right) C^{k l p q} E_{p q}\right] \cdot d V\right) \dot{h}+ \\
& +\frac{\partial^{2}}{\partial h^{2}}\left(\int_{(B)} \delta \dot{u}_{m ; k}\left(\delta_{l}^{m}+u_{; l}^{m}\right) C^{k l p q} E_{p q} \mathrm{~d} V\right) \dot{h}^{2}+ \\
& +\frac{\partial}{\partial h}\left(\int_{(B)} \delta \dot{u}_{m ; k}\left(\delta_{l}^{m}+u_{; l}^{m}\right) C^{k l p q} E_{p q} \mathrm{~d} V\right) \ddot{h}- \\
& -\sum_{\mathrm{N}=\mathrm{I}}^{\mathrm{II}}\left[\ddot{p}_{\mathrm{N}} \int_{\left(A_{\mathrm{tN}}\right)} \widetilde{p}_{\mathrm{N} 0} \delta \dot{u}^{k} Q_{k}^{p} \mathrm{~d} A_{p}+2 \dot{p}_{\mathrm{N}} \int_{\left(A_{\mathrm{tN}}\right)} \widetilde{p}_{\mathrm{N} 0} \delta \dot{u}^{k} \dot{Q}_{k}^{p} \mathrm{~d} A_{p}+\right. \\
& +p_{\mathrm{N}} \int_{\left(A_{\mathrm{tN}}\right)} \widetilde{p}_{\mathrm{N} 0} \delta \dot{u}^{k} \ddot{Q}_{k}^{p} \mathrm{~d} A_{p}+2 \dot{p}_{\mathrm{N}} \frac{\partial}{\partial h}\left(\int_{\left(A_{\mathrm{tN}}\right)} \widetilde{p}_{\mathrm{N} 0} \delta \dot{u}^{k} Q_{k}^{p} \mathrm{~d} A_{p}\right) \dot{h}+ \\
& +2 p_{\mathrm{N}} \frac{\partial}{\partial h}\left(\int_{\left(A_{\mathrm{tN}}\right)} \widetilde{p}_{\mathrm{N} 0} \delta \dot{u}^{k} \dot{Q}_{k}^{p} \mathrm{~d} A_{p}\right) \dot{h}+p_{\mathrm{N}} \frac{\partial^{2}}{\partial h^{2}}\left(\int_{\left(A_{\mathrm{tN}}\right)} \widetilde{p}_{\mathrm{N} 0} \delta \dot{u}^{k} Q_{k}^{p} \mathrm{~d} A_{p}\right) \dot{h}^{2}+ \\
& \left.+p_{\mathrm{N}} \frac{\partial}{\partial h}\left(\int_{\left(A_{\mathrm{tN}}\right)} \widetilde{p}_{\mathrm{N} 0} \delta \dot{u}^{k} Q_{k}^{p} \mathrm{~d} A_{p}\right) \ddot{h}\right]=0 .
\end{aligned}
$$

The following equation is valid only under the condition that the imperfection parameter is constant, i.e., $h=h_{0}=$ constant:

$$
\int_{(B)} \delta \dot{u}_{m ; k}\left[\left(\delta_{l}^{m}+u_{; l}^{m}\right) C^{k l p q} E_{p q}\right]^{\cdots} \mathrm{d} V=\sum_{\mathrm{N}=\mathrm{I}}^{\mathrm{II}}\left(p_{\mathrm{N}} \int_{\left(A_{\mathrm{tN}}\right)} \widetilde{p}_{\mathrm{N} 0} \delta \dot{u}^{k} Q_{k}^{p} \mathrm{~d} A_{p}\right)^{\cdots} .
$$

In the sequel we shall detail the previous equilibrium equations by assuming that the imperfection parameter $h=h_{0}=$ constant. We get the following relations:

$$
\begin{aligned}
& \int_{(B)} \delta \dot{u}_{m ; k}\left[\left(\delta_{l}^{m}+u_{; l}^{m}\right) \cdot C^{k l p q} E_{p q}+\left(\delta_{l}^{m}+u_{; l}^{m}\right) C^{k l p q}\left(\delta_{p}^{s}+u_{; p}^{s}\right) \dot{u}_{s ; q}\right] \mathrm{d} V- \\
& -\sum_{\mathrm{N}=\mathrm{I}}^{\mathrm{II}}\left[p_{\mathrm{N}} \int_{\left(A_{\mathrm{tN}}\right)} \widetilde{p}_{\mathrm{N} 0} \delta \dot{u}^{k} e_{k l m} e^{p q r}\left(\delta_{q}^{l}+u_{; q}^{l}\right) \dot{u}_{; r}^{m} \mathrm{~d} A_{p}+\dot{p}_{\mathrm{N}} \int_{\left(A_{\mathrm{tN}}\right)} \widetilde{p}_{\mathrm{N} 0} \delta \dot{u}^{k} Q_{k}{ }^{p} \mathrm{~d} A_{p}\right]=0, \\
& \int_{(B)} \delta \dot{u}_{m ; k}\left[\left(\delta_{l}^{m}+u_{; l}^{m}\right)^{\cdot \cdot} C^{k l p q} E_{p q}+2\left(\delta_{l}^{m}+u_{; l}^{m}\right) C^{k l p q}\left(\delta_{p}^{s}+u_{; p}^{s}\right) \dot{u}_{s ; q}\right] \mathrm{d} V+
\end{aligned}
$$




$$
\begin{aligned}
& +\int_{(B)} \delta \dot{u}_{m ; k}\left(\delta_{l}^{m}+u_{; l}^{m}\right) C^{k l p q}\left[\left(\delta_{l}^{m}+u_{; l}^{m}\right) \dot{u}_{s ; q}+\left(\delta_{l}^{m}+u_{; l}^{m}\right) \ddot{u}_{s ; q}\right] \mathrm{d} V- \\
& -\sum_{\mathrm{N}=\mathrm{I}}^{\mathrm{II}} p_{\mathrm{N}} \int_{\left(A_{\mathrm{tN}}\right)} \widetilde{p}_{\mathrm{N} 0} \delta \dot{u}^{k} e_{k l m} e^{p q r}\left[\left(\delta_{q}^{l}+u_{; q}^{l}\right) \dot{u}_{; r}^{m}+\left(\delta_{q}^{l}+u_{; q}^{l}\right) \ddot{u}_{; r}^{m}\right] \mathrm{d} A_{p}- \\
& -\sum_{\mathrm{N}=\mathrm{I}}^{\mathrm{II}}\left[2 \dot{p}_{\mathrm{N}} \int_{\left(A_{\mathrm{tN}}\right)} \widetilde{p}_{\mathrm{N} 0} \delta \dot{u}^{k} e_{k l m} e^{p q r}\left(\delta_{q}^{l}+u_{; q}^{l}\right) \dot{u}_{; r}^{m} \mathrm{~d} A_{p}+\right. \\
& \left.+\ddot{p}_{\mathrm{N}} \int_{\left(A_{\mathrm{tN}}\right)} \tilde{p}_{\mathrm{N} 0} \delta \dot{u}^{k} Q_{k}^{p} \mathrm{~d} A_{p}\right]=0, \\
& \int_{(B)} \delta \dot{u}_{m ; k}\left[\left(\delta_{l}^{m}+u_{; l}^{m}\right)^{\cdots} C^{k l p q} E_{p q}+3\left(\delta_{l}^{m}+u_{; l}^{m}\right)^{\cdot \cdot} C^{k l p q}\left(\delta_{p}^{s}+u_{; p}^{s}\right) \dot{u}_{s ; q}\right] \mathrm{d} V+ \\
& +\int_{(B)} \delta \dot{u}_{m ; k} 3\left(\delta_{l}^{m}+u_{; l}^{m}\right) \cdot C^{k l p q}\left[\left(\delta_{p}^{s}+u_{; p}^{s}\right)^{\cdot} \dot{u}_{s ; q}+\left(\delta_{p}^{s}+u_{; p}^{s}\right) \ddot{u}_{s ; q}\right] \mathrm{d} V+ \\
& +\int_{(B)} \delta \dot{u}_{m ; k}\left(\delta_{l}^{m}+u_{; l}^{m}\right) C^{k l p q}\left[\left(\delta_{p}^{s}+u_{; p}^{s}\right)^{\cdot \cdot} \dot{u}_{s ; q}+2\left(\delta_{p}^{s}+u_{; p}^{s}\right)^{\cdot} \ddot{u}_{s ; q}+\left(\delta_{p}^{s}+u_{; p}^{s}\right) \dddot{u}_{s ; q}\right] \mathrm{d} V- \\
& -\sum_{\mathrm{N}=\mathrm{I}}^{\mathrm{II}} p_{\mathrm{N}} \int_{\left(A_{\mathrm{tN}}\right)} \widetilde{p}_{\mathrm{N} 0} \delta \dot{u}^{k} e_{k l m} e^{p q r}\left[\left(\delta_{q}^{l}+u_{; q}^{l}\right)^{\cdot \cdot} \dot{u}_{; r}^{m}+2\left(\delta_{q}^{l}+u_{; q}^{l}\right)^{\cdot} \ddot{u}_{; r}^{m}+\left(\delta_{q}^{l}+u_{; q}^{l}\right) \dddot{u}_{; r}^{m}\right] \mathrm{d} A_{p}- \\
& -\sum_{\mathrm{N}=\mathrm{I}}^{\mathrm{II}} 3 \dot{p}_{\mathrm{N}} \int_{\left(A_{\mathrm{tN}}\right)} \widetilde{p}_{\mathrm{N} 0} \delta \dot{u}^{k} e_{k l m} e^{p q r}\left[\left(\delta_{q}^{l}+u_{; q}^{l}\right) \dot{u}_{; r}^{m}+\left(\delta_{q}^{l}+u_{; q}^{l}\right) \ddot{u}_{; r}^{m}\right] \mathrm{d} A_{p}- \\
& -\sum_{\mathrm{N}=\mathrm{I}}^{\mathrm{II}} 3 \ddot{p}_{\mathrm{N}} \int_{\left(A_{\mathrm{tN}}\right)} \widetilde{p}_{\mathrm{N} 0} \delta \dot{u}^{k} e_{k l m} e^{p q r}\left(\delta_{q}^{l}+u_{; q}^{l}\right) \dot{u}_{; r}^{m} \mathrm{~d} A_{p}- \\
& -\sum_{\mathrm{N}=\mathrm{I}}^{\mathrm{II}} \dddot{p}_{\mathrm{N}} \int_{\left(A_{\mathrm{tN}}\right)} \widetilde{p}_{\mathrm{N} 0} \delta \dot{u}^{k} Q_{k}^{p} \mathrm{~d} A_{p}=0 .
\end{aligned}
$$

3.3. Power of the inner and external forces in the equilibrium state $(\bar{B})$ of the body. Since $\tau=0$ in the equilibrium state $(\bar{B})$ of the body the power sought is of the form

$$
P=\sum_{\mathrm{N}=\mathrm{I}}^{\mathrm{II}} p_{\mathrm{N}} \int_{\left(A_{\mathrm{tN}}\right)} \tilde{p}_{\mathrm{N} 0}\left(\dot{u}^{k} Q_{k}^{p}\right) \mathrm{d} A_{p}-\int_{(B)}\left(\dot{E}_{k l} C^{k l p q} E_{p q}\right) \mathrm{d} V,
$$

As regards the intermediate states $0<\tau<\Delta \tau\left(p_{\mathrm{N}}=\right.$ const $\mathrm{N}=\mathrm{I}$, II $)$ we have

$$
\begin{aligned}
& P(\tau)=P+\dot{P} \tau+\frac{1}{2} \ddot{P} \tau^{2}+\frac{1}{6} \dddot{P} \tau^{3}+\cdots= \\
& =P+\left\{\sum_{\mathrm{N}=\mathrm{I}}^{\mathrm{II}} p_{\mathrm{N}} \int_{\left(A_{\mathrm{tN}}\right)} \widetilde{p}_{\mathrm{N} 0}\left(\dot{u}^{k} Q_{k}^{p}\right) \cdot \mathrm{d} A_{p}-\int_{(B)}\left(\dot{E}_{k l} C^{k l p q} E_{p q}\right) \mathrm{d} V+\right.
\end{aligned}
$$




$$
\begin{aligned}
& \left.+\left[\sum_{\mathrm{N}=\mathrm{I}}^{\mathrm{II}} p_{\mathrm{N}} \frac{\partial}{\partial h} \int_{\left(A_{\mathrm{tN}}\right)} \widetilde{p}_{\mathrm{N} 0}\left(\dot{u}^{k} Q_{k}^{p}\right) \mathrm{d} A_{p}-\frac{\partial}{\partial h} \int_{(B)}\left(\dot{E}_{k l} C^{k l p q} E_{p q}\right) \mathrm{d} V\right] \dot{h}\right\} \tau+ \\
& +\frac{1}{2}\left\{\sum_{\mathrm{N}=\mathrm{I}}^{\mathrm{II}} p_{\mathrm{N}} \int_{\left(A_{\mathrm{tN}}\right)} \widetilde{p}_{\mathrm{N} 0}\left(\dot{u}^{k} Q_{k}^{p}\right)^{. \cdot} \mathrm{d} A_{p}-\int_{(B)}\left(\dot{E}_{k l} C^{k l p q} E_{p q}\right)^{* \cdot} \mathrm{d} V+\right. \\
& +2\left[\sum_{\mathrm{N}=\mathrm{I}}^{\mathrm{II}} p_{\mathrm{N}} \frac{\partial}{\partial h} \int_{\left(A_{\mathrm{tN}}\right)} \widetilde{p}_{\mathrm{N} 0}\left(\dot{u}^{k} Q_{k}{ }^{p}\right) \mathrm{d} A_{p}-\frac{\partial}{\partial h} \int_{(B)}\left(\dot{E}_{k l} C^{k l p q} E_{p q}\right) \dot{\mathrm{d} V}\right] \dot{h}+ \\
& +\left[\sum_{\mathrm{N}=\mathrm{I}}^{\mathrm{II}} p_{\mathrm{N}} \frac{\partial^{2}}{\partial h^{2}} \int_{\left(A_{\mathrm{tN})}\right.} \widetilde{p}_{\mathrm{N} 0}\left(\dot{u}^{k} Q_{k}^{p}\right) \mathrm{d} A_{p}-\frac{\partial^{2}}{\partial h^{2}} \int_{(B)}\left(\dot{E}_{k l} C^{k l p q} E_{p q}\right) \mathrm{d} V\right] \dot{h}^{2}+ \\
& \left.+\left[\sum_{\mathrm{N}=\mathrm{I}}^{\mathrm{II}} p_{\mathrm{N}} \frac{\partial}{\partial h} \int_{\left(A_{\mathrm{tN}}\right)} \widetilde{p}_{\mathrm{N} 0}\left(\dot{u}^{k} Q_{k}^{p}\right) \mathrm{d} A_{p}-\frac{\partial}{\partial h} \int_{(B)}\left(\dot{E}_{k l} C^{k l p q} E_{p q}\right) \mathrm{d} V\right] \ddot{h}\right\} \tau^{2}+\cdots
\end{aligned}
$$

\subsection{The work $\Delta L$ of the inner and external forces in the equilibrium state $(\bar{B})$ of the body.}

3.4.1. Work done on the kinematically admissible increment of the displacement field. The kinematically admissible displacement field are determined by equation $(3.17 \mathrm{a})_{2}$. The work done by the inner and external forces can be obtained by integrating equation 3.51 with respect to time:

$$
\Delta L_{k i n}=\int_{\tau=0}^{\Delta \tau} P(\tau) \mathrm{d} \tau=\Delta L_{k i n}^{(1)}+\Delta L_{k i n}^{(2)}+\Delta L_{k i n}^{(3)}+\cdots
$$

By using the finite element formalism we may write

$$
\begin{gathered}
\Delta L_{k i n}^{(1)}=-\dot{\mathbf{t}}_{i}^{\mathrm{T}}\left(\mathbf{b}_{i}-\sum_{\mathrm{N}=\mathrm{I}}^{\mathrm{II}} p_{\mathrm{N}} \mathbf{g}_{\mathrm{N} i}\right) \Delta \tau=0 \\
\Delta L_{k i n}^{(2)}=-\left[\ddot{\mathbf{t}}_{i}^{\mathrm{T}}\left(\mathbf{b}_{i}-\sum_{\mathrm{N}=\mathrm{I}}^{\mathrm{II}} p_{\mathrm{N}} \mathbf{g}_{\mathrm{N} i}\right)+\dot{\mathbf{t}}_{i}^{\mathrm{T}}\left(\mathbf{K}_{i j}^{\mathrm{L}}+\mathbf{K}_{i j}^{\mathrm{G}}-\sum_{\mathrm{N}=\mathrm{I}}^{\mathrm{II}} \mathbf{K}_{\mathrm{N} i j}^{\mathrm{C}} p_{\mathrm{N}}\right) \dot{\mathbf{t}}_{j}+\right. \\
\left.+\dot{\mathbf{t}}_{i}^{\mathrm{T}}\left(\frac{\partial \mathbf{b}_{i}}{\partial h}-\sum_{\mathrm{N}=\mathrm{I}}^{\mathrm{II}} \frac{\partial \mathbf{g}_{\mathrm{N} i}}{\partial h} p_{\mathrm{N}}\right) \dot{h}\right] \frac{\Delta \tau^{2}}{2}, \\
\Delta L_{k i n}^{(3)}=-\left\{\begin{array}{c}
\left.\dddot{\mathbf{t}}_{i}^{\mathrm{T}}\left(\mathbf{b}_{i}-\sum_{\mathrm{N}=\mathrm{I}}^{\mathrm{II}} p_{\mathrm{N}} \mathbf{g}_{\mathrm{N} i}\right)+2.53\right) \\
+\ddot{\mathbf{t}}_{i}^{\mathrm{T}}\left(\mathbf{K}_{i j}^{\mathrm{L}}+\mathbf{K}_{i j}^{\mathrm{G}}-\sum_{\mathrm{N}=\mathrm{I}}^{\mathrm{II}} \mathbf{K}_{\mathrm{N} i j}^{\mathrm{C}} p_{\mathrm{N}}\right) \dot{\mathbf{t}}_{j}+ \\
\left.\mathbf{K}_{i j}^{\mathrm{G}}-\sum_{\mathrm{N}=\mathrm{I}}^{\mathrm{II}} \mathbf{K}_{\mathrm{N} i j}^{\mathrm{C}} p_{\mathrm{N}}\right) \ddot{\mathbf{t}}_{j}+\dot{\mathbf{t}}_{i}^{\mathrm{T}}\left(2 \mathbf{J}_{i k j}+\mathbf{H}_{i j k}-\sum_{\mathrm{N}=\mathrm{I}}^{\mathrm{II}} \mathbf{M}_{\mathrm{N} i k j} p_{\mathrm{N}}\right) \dot{\mathbf{t}}_{k} \dot{\mathbf{t}}_{j}+
\end{array}\right.
\end{gathered}
$$




$$
\begin{array}{r}
+2\left[\ddot{\mathbf{t}}_{i}^{\mathrm{T}}\left(\frac{\partial \mathbf{b}_{i}}{\partial h}-\sum_{\mathrm{N}=\mathrm{I}}^{\mathrm{II}} \frac{\partial \mathbf{g}_{\mathrm{N} i}}{\partial h} p_{\mathrm{N}}\right) \dot{h}+\dot{\mathbf{t}}_{i}^{\mathrm{T}} \frac{\partial}{\partial h}\left(\mathbf{K}_{i j}^{\mathrm{L}}+\mathbf{K}_{i j}^{\mathrm{G}}-\sum_{\mathrm{N}=\mathrm{I}}^{\mathrm{II}} \mathbf{K}_{\mathrm{N} i j}^{\mathrm{C}} p_{\mathrm{N}}\right) \dot{\mathbf{t}}_{j} \dot{h}\right]+ \\
\left.+\dot{\mathbf{t}}_{i}^{\mathrm{T}}\left(\frac{\partial^{2} \mathbf{b}_{i}}{\partial h^{2}}-\sum_{\mathrm{N}=\mathrm{I}}^{\mathrm{II}} \frac{\partial^{2} \mathbf{g}_{\mathrm{N} i}}{\partial h^{2}} p_{\mathrm{N}}\right) \dot{h}^{2}+\dot{\mathbf{t}}_{i}^{\mathrm{T}}\left(\frac{\partial \mathbf{b}_{i}}{\partial h}-\sum_{\mathrm{N}=\mathrm{I}}^{\mathrm{II}} \frac{\partial \mathbf{g}_{\mathrm{N} i}}{\partial h} p_{\mathrm{N}}\right) \ddot{h}\right\} \frac{\Delta \tau^{3}}{6} .
\end{array}
$$

Equation 3.53 reflects the equilibrium of state $(\bar{B})$ of the body.

3.4.2. Work on the displacement increment taken on the equilibrium path. Consider the equilibrium path to the state $(\bar{B}+\Delta \bar{B})$ determined by the load increment $(3.17 \mathrm{~d})_{2}$ and the geometrical imperfection parameter. The work of the inner and external forces on this path can be calculated by using equations (3.53)- (3.55) provided that the equilibrium equations (3.19), (3.41) and (3.42) are also taken into account:

$$
\Delta L_{\text {path }}=\Delta L^{(1)}+\Delta L^{(2)}+\Delta L^{(3)}+\cdots
$$

Here $\Delta L^{(1)}=0$ and

$$
\begin{aligned}
& \Delta L^{(2)}=-\sum_{\mathrm{N}=\mathrm{I}}^{\mathrm{II}}\left[\dot{\mathbf{t}}_{i}^{\mathrm{T}} \mathbf{g}_{\mathrm{N} i} \dot{p}_{\mathrm{N}}\right] \frac{\Delta \tau^{2}}{2}=-\left[\sum_{\mathrm{N}=\mathrm{I}}^{\mathrm{II}} \dot{p}_{\mathrm{N}} \int_{\left(A_{\mathrm{tN}}\right)} \widetilde{p}_{\mathrm{N} 0}\left(\dot{u}^{k} Q_{k}{ }^{p}\right) \mathrm{d} A_{p}\right] \frac{\Delta \tau^{2}}{2} \\
& \Delta L^{(3)}=-\sum_{\mathrm{N}=\mathrm{I}}^{\mathrm{II}}\left[\left(\ddot{\mathbf{t}}_{i}^{\mathrm{T}} \mathbf{g}_{\mathrm{N} i}+\dot{\mathbf{t}}_{i}^{\mathrm{T}} \mathbf{K}_{\mathrm{N} i j}^{\mathrm{C}} \dot{\mathbf{t}}_{j}\right) 2 \dot{p}_{\mathrm{N}}+\dot{\mathbf{t}}_{i}^{\mathrm{T}} \mathbf{g}_{\mathrm{N} i} \ddot{p}_{\mathrm{N}}+\dot{\mathbf{t}}_{i}^{\mathrm{T}} 2 \frac{\partial \mathbf{g}_{\mathrm{N} i}}{\partial h} \dot{p}_{\mathrm{N}} \dot{h}\right] \frac{\Delta \tau^{3}}{6}= \\
& =-\sum_{\mathrm{N}=\mathrm{I}}^{\mathrm{II}}\left[2 \dot{p}_{\mathrm{N}} \int_{\left(A_{\mathrm{tN}}\right)} \widetilde{p}_{\mathrm{N} 0}\left(\ddot{u}^{k} Q_{k}^{p}+\dot{u}^{k} \dot{Q}_{k}^{p}\right) \mathrm{d} A_{p}+\ddot{p}_{\mathrm{N}} \int_{\left(A_{\mathrm{tN}}\right)} \widetilde{p}_{\mathrm{N} 0}\left(\dot{u}^{k} Q_{k}^{p}\right) \mathrm{d} A_{p}+\right. \\
& \left.+2 \dot{p}_{\mathrm{N}} \dot{h} \frac{\partial}{\partial h} \int_{\left(A_{\mathrm{tN}}\right)} \widetilde{p}_{\mathrm{N} 0}\left(\dot{u}^{k} Q_{k}^{p}\right) \mathrm{d} A_{p}\right] \frac{\Delta \tau^{3}}{6}, \\
& \Delta L^{(4)}=-\sum_{\mathrm{N}=\mathrm{I}}^{\mathrm{II}}\left[3 \dot{p}_{\mathrm{N}} \int_{\left(A_{\mathrm{tN}}\right)} \widetilde{p}_{\mathrm{N} 0}\left(\dddot{u}^{k} Q_{k}^{p}+2 \ddot{u}^{k} \dot{Q}_{k}^{p}+\dot{u}^{k} \ddot{Q}_{k}^{p}\right) \mathrm{d} A_{p}+\right. \\
& +3 \ddot{p}_{\mathrm{N}} \int_{\left(A_{\mathrm{tN}}\right)} \widetilde{p}_{\mathrm{N} 0}\left(\ddot{u}^{k} Q_{k}^{p}+\dot{u}^{k} \dot{Q}_{k}^{p}\right) \mathrm{d} A_{p}+\dddot{p}_{\mathrm{N}} \int_{\left(A_{\mathrm{tN}}\right)} \widetilde{p}_{\mathrm{N} 0}\left(\dot{u}^{k} Q_{k}^{p}\right) \mathrm{d} A_{p}+ \\
& +6 \dot{p}_{\mathrm{N}} \dot{h} \frac{\partial}{\partial h} \int_{\left(A_{\mathrm{tN}}\right)} \widetilde{p}_{\mathrm{N} 0}\left(\dot{u}^{k} Q_{k}^{p}\right)^{\cdot} \mathrm{d} A_{p}+3 \dot{p}_{\mathrm{N}} \dot{h}^{2} \frac{\partial^{2}}{\partial h^{2}} \int_{\left(A_{\mathrm{tN}}\right)} \widetilde{p}_{\mathrm{N} 0}\left(\dot{u}^{k} Q_{k}^{p}\right) \mathrm{d} A_{p}+ \\
& \left.+3 \ddot{p}_{\mathrm{N}} \dot{h} \frac{\partial}{\partial h} \int_{\left(A_{\mathrm{tN}}\right)} \widetilde{p}_{\mathrm{N} 0}\left(\dot{u}^{k} Q_{k}^{p}\right) \mathrm{d} A_{p}+3 \dot{p}_{\mathrm{N}} \ddot{h} \frac{\partial}{\partial h} \int_{\left(A_{\mathrm{tN}}\right)} \widetilde{p}_{\mathrm{N} 0}\left(\dot{u}^{k} Q_{k}^{p}\right) \mathrm{d} A_{p}\right] \frac{\Delta \tau^{4}}{24} .
\end{aligned}
$$

$\Delta L^{(3)}$ and $\Delta L^{(4)}$ can be given in another form as well:

$$
\Delta L^{(3)}=\left(\Delta L^{(2)}\right) \cdot \frac{\Delta \tau}{3}-\sum_{\mathrm{N}=\mathrm{I}}^{\mathrm{II}} \dot{p}_{\mathrm{N}}\left[\int_{\left(A_{\mathrm{tN}}\right)} \tilde{p}_{\mathrm{N} 0}\left(\dot{u}^{k} Q_{k}^{p}\right) \mathrm{d} A_{p}\right]^{\cdot} \frac{\Delta \tau^{3}}{6},
$$




$$
\begin{aligned}
& \Delta L^{(4)}=\left(\Delta L^{(3)}\right) \cdot \frac{\Delta \tau}{4}- \\
& -\sum_{\mathrm{N}=\mathrm{I}}^{\mathrm{II}}\left\{\dot{p}_{\mathrm{N}} \int_{\left(A_{\mathrm{tN}}\right)} \widetilde{p}_{\mathrm{N} 0}\left(\dot{u}^{k} Q_{k}^{p}\right)^{\cdot \cdot} \mathrm{d} A_{p}+\dddot{p}_{\mathrm{N}} \int_{\left(A_{\mathrm{tN}}\right)} \widetilde{p}_{\mathrm{N} 0}\left(\dot{u}^{k} Q_{k}^{p}\right) \mathrm{d} A_{p}+\right. \\
& +\dot{p}_{\mathrm{N}} \dot{h}^{2} \frac{\partial^{2}}{\partial h^{2}} \int_{\left(A_{\mathrm{tN}}\right)} \widetilde{p}_{\mathrm{N} 0}\left(\dot{u}^{k} Q_{k}^{p}\right) \mathrm{d} A_{p}+4 \dot{p}_{\mathrm{N}} \dot{h} \frac{\partial}{\partial h} \int_{\left(A_{\mathrm{tN}}\right)} \widetilde{p}_{\mathrm{N} 0}\left(\dot{u}^{k} Q_{k}^{p}\right) \cdot \mathrm{d} A_{p}+ \\
& \left.+\left(\dot{p}_{\mathrm{N}} \dot{h}\right) \frac{\partial}{\partial h} \int_{\left(A_{\mathrm{tN})}\right.} \widetilde{p}_{\mathrm{N} 0}\left(\dot{u}^{k} Q_{k}^{p}\right) \mathrm{d} A_{p}\right\} \frac{\Delta \tau^{4}}{24} .
\end{aligned}
$$

Time derivatives $\left(\Delta L^{(3)}\right)^{\circ},\left(\Delta L^{(3)}\right)^{\cdot}$ in equations $(3.60)$ ) and $(3.61)$ imply that the surface integrals depend on the geometrical imperfection parameter $h$ as well.

3.5. Stability criteria. The equilibrium state of a solid body is stable if the work done by the inner and external forces of the equilibrium state on any kinematically admissible and sufficiently small displacement increment is negative.

This axiomatic statement is based on the following theorem of mechanics: Assume that the equilibrium state of the solid body is disturbed in such a way that it has some but low kinetic energy (this is the case of a small disturbation). If the kinetic energy is decreased on any kinematically admissible displacement increment (the work done by the inner and external forces of the equilibrium state is negative) then the motion of the solid body remains in a small neighborhood of the equilibrium state.

In the opposite case, i.e., when the work done by the inner and external forces of the equilibrium state is zero or positive the equilibrium state is not stable.

It follows from the above definition of stability that the work $\Delta L_{\mathrm{kin}}$ is applicable to characterizing the stability of equilibrium both in pre-critical (before stability loss) and post-critical states of the body.

The numerical investigations should be carried out on the equilibrium paths.

A summary for the stability criteria can be given on the basis of all that has been said above by utilizing the work increment $\Delta L_{\text {path }}=\Delta L^{(2)}+\Delta L^{(3)}+\Delta L^{(4)} \ldots$ which can be calculated with equations (3.57)-(3.59):

The equilibrium state at a point on the equilibrium path is

$$
\left\{\begin{array}{l}
\text { stable } \\
\text { critical } \\
\text { instable }
\end{array}\right\} \text { if }\left\{\begin{array}{l}
\Delta L^{(2)}<0 \\
\Delta L^{(2)}=0 \\
\Delta L^{(2)}>0
\end{array}\right\} \text {. }
$$

The critical point is a

$$
\left\{\begin{array}{l}
\text { limit point } \\
\text { branching point }
\end{array}\right\} \text { if }\left\{\begin{array}{c}
\Delta L^{(3)} \neq 0 \\
\Delta L^{(3)}=0, \text { but } \Delta L^{(4)} \neq 0
\end{array}\right\} .
$$

We speak about a total stability analysis if the investigations are carried out not only at a few points on the equilibrium path but in an interval of the problem parameters which includes the pre-critical and post-critical states of the body as well. 


\section{Characteristics of Critical states}

\subsection{Definitions.}

4.1.1. Equilibrium conditions. According to equations $3.22 \sqrt{3.23})_{2}$ and $(3.41)$ in an equilibrium state it holds that

$$
\begin{gathered}
\mathbf{A}_{i}=\mathbf{b}_{i}-\mathbf{g}_{\mathrm{I} i} p_{\mathrm{I}}-\mathbf{g}_{\mathrm{II} i} p_{\mathrm{II}}=0 \\
\dot{\mathbf{A}}_{i}=\mathbf{K}_{i j} \dot{\mathbf{t}}_{j}-\mathbf{g}_{\mathrm{I} i} \dot{p}_{\mathrm{I}}-\mathbf{g}_{\mathrm{II} i} \dot{p}_{\mathrm{II}}+\frac{\partial \mathbf{A}_{i}}{\partial h} \dot{h}=0,
\end{gathered}
$$

where

$$
\mathbf{K}_{i j}=\frac{\partial \mathbf{A}_{i}}{\partial \mathbf{t}_{j}}=\mathbf{K}_{i j}^{\mathrm{L}}+\mathbf{K}_{i j}^{\mathrm{G}}-\mathbf{K}_{\mathrm{I} i j}^{\mathrm{C}} p_{\mathrm{I}}-\mathbf{K}_{\mathrm{II} i j}^{\mathrm{C}} p_{\mathrm{II}} .
$$

In a critical equilibrium state equation 4.2 is undetermined hence

$$
\operatorname{det} \mathbf{K}_{i j}^{\mathrm{cr}}=0 \text {, }
$$

from where it follows that

$$
\mathbf{K}_{i j}^{\mathrm{cr}} \mathbf{u}_{j}=0, \quad \mathbf{v}_{i}^{\mathrm{T}} \mathbf{K}_{i j}^{\mathrm{cr}}=0,
$$

in which $\mathbf{u}_{j}$ and $\mathbf{v}_{i}$ are the right and left eigenvectors, respectively. It is assumed that they belong to the same simple eigenvalue (to an eigenvalue of multiplicity one).

As is well known $\mathbf{u}_{j}=\mathbf{v}_{j}$ if $\mathbf{K}_{i j}$ is symmetric.

In the present case the loads are non-conservative deformation independent surface loads hence the matrix $\mathbf{K}_{i j}$ is not symmetric. Consequently $\mathbf{u}_{j} \neq \mathbf{v}_{j}$.

After multiplying equation (4.1) by $\mathbf{v}_{i}$ we get on the basis of 4.3$)_{2}$ that

$$
\mathbf{v}_{i}^{\mathrm{T}} \mathbf{g}_{\mathrm{I} i}^{\mathrm{cr}} \dot{p}_{\mathrm{I}}^{\mathrm{cr}}+\mathbf{v}_{i}^{\mathrm{T}} \mathbf{g}_{\mathrm{II} i}^{\mathrm{cr}} \dot{p}_{\mathrm{II}}^{\mathrm{cr}}-\mathbf{v}_{i}^{\mathrm{T}}\left(\frac{\partial \mathbf{A}_{i}}{\partial h}\right)^{\mathrm{cr}} \dot{h}^{\mathrm{kr}}=0 .
$$

REMARK 4.1: According to Subsection 3.1 $p_{\text {II }}$ can be either the parameter of a supplementary load or a load imperfection parameter (load disturbance).

4.1.2. The geometrical imperfection parameter $h=$ constant. According to the definition of Subsection 3.2 .3 equilibrium paths can be obtained in the cases $h=$ constant or $p_{\mathrm{II}}=$ constant. In the sequel these two cases are considered one by one.

Assume that $h=$ constant $(\dot{h}=0)$ and independently of each other either $p_{I}$ varies $\left(p_{I I}=\right.$ constant $)$ or $p_{I I}$ varies $\left(p_{I}=\right.$ constant $)$. Assume further that (4.4) is satisfied. Then the critical points on the equilibrium paths can be classified as follows:

- the critical point is a limit point if

$$
\dot{p}_{\mathrm{I}}^{\text {cr }}=0, \dot{p}_{\mathrm{II}}=0 \quad \text { or } \quad \dot{p}_{\mathrm{II}}^{\text {cr }}=0, \dot{p}_{\mathrm{I}}=0,
$$

- the critical point is a branching point if

$$
\mathbf{v}_{i}^{\mathrm{T}} \mathbf{g}_{\mathrm{I} i}^{\mathrm{cr}}=0, \dot{p}_{\mathrm{II}}=0 \quad \text { or } \quad \mathbf{v}_{i}^{\mathrm{T}} \mathbf{g}_{\mathrm{II} i}^{\mathrm{cr}}=0, \dot{p}_{\mathrm{I}}=0 .
$$

Let us return now to the conditions the critical points should meet - these are detailed in Subsection 3.5. According to equations (3.57), 4.5), 4.6 and Remark 4.2 condition

$$
\Delta L^{(2) \mathrm{cr}}=-\left(\mathbf{v}_{i}^{\mathrm{T}} \mathbf{g}_{\mathrm{I} i}^{\mathrm{cr}} \dot{p}_{\mathrm{I}}^{\mathrm{cr}}+\mathbf{v}_{i}^{\mathrm{T}} \mathbf{g}_{\mathrm{II} i}^{\mathrm{cr}} \dot{p}_{\mathrm{II}}^{\mathrm{cr}}\right) \frac{\Delta \tau^{2}}{2}=0
$$


for the critical point on the equilibrium path $(\dot{h}=0 !)$ is satisfied at the limit point.

REMARK 4.2: Equation 4.5 is satisfied at a limit point. Hence it follows from equation (4.2) of the critical equilibrium state $(\dot{h}=0$ !) that

$$
\mathbf{K}_{i j}^{\mathrm{cr}} \dot{\mathbf{t}}_{j}^{\mathrm{cr}}=0 \text {. }
$$

Equation 4.7 does not determine uniquely the order of magnitude of $\dot{\mathbf{t}}_{j}^{\text {cr }}$ since $\dot{\mathbf{t}}_{j}^{\text {cr }}$ is obtained from $\mathbf{u}_{j}$ by multiplying the latter with a scalar. This follows from detailing equation 4.7

$$
\mathbf{K}_{i j}^{\mathrm{cr}} \dot{\mathbf{t}}_{j}^{\mathrm{cr}}=\left[\begin{array}{ccccc} 
& & & \vdots & \\
& \mathbf{K}_{\alpha \beta}^{\mathrm{cr}} & & \vdots & \mathbf{k}_{\alpha m}^{\mathrm{cr}} \\
& & & \vdots & \\
\cdots & \ldots & \cdots & \cdots & \ldots \\
& \mathbf{k}_{m \beta}^{\mathrm{Tcr}} & & \vdots & k_{m m}^{\mathrm{cr}}
\end{array}\right]\left[\begin{array}{c}
\vdots \\
\dot{\mathbf{t}}_{\beta}^{\mathrm{cr}} \\
\vdots \\
\cdots \\
\dot{t}_{m}^{\mathrm{cr}}
\end{array}\right]=0,
$$

where $\alpha, \beta=1,2, \cdots, m-1$ and $m$ is the number of freedom a $\left(k_{m m}^{\mathrm{cr}}\right.$ is a scalar!). Equation 4.8 can also be given in the form

$$
\left[\begin{array}{ccccc} 
& & & \vdots & \\
& \mathbf{K}_{\alpha \beta}^{\mathrm{cr}} & & \vdots & \mathbf{k}_{\alpha m}^{\mathrm{cr}} \\
& & & \vdots & \\
\cdots & \ldots & \cdots & \cdots & \cdots \\
& \mathbf{k}_{m \beta}^{\mathrm{Tcr}} & & \vdots & k_{m m}^{\mathrm{cr}}
\end{array}\right]\left[\begin{array}{c}
\vdots \\
\dot{\mathbf{t}}_{\beta}^{\mathrm{cr}} \\
\dot{t}_{m}^{\mathrm{cr}} \\
\vdots \\
\cdots \\
1
\end{array}\right]=0
$$

which proves the above statement.

REMARK 4.3: Though $m$ is the number of freedom, it can be the subscript for any non-zero displacement component in the corresponding equations.

REMARK 4.4: All that has been said above is valid independently of the fact what quantity is the governing parameter: that can be either a displacement component or a load.

4.1.3. The load imperfection parameter $p_{\mathrm{II}}=$ constant. Assume that $p_{I I}=$ constant $\left(\dot{p}_{I I}=0\right)$ and independently of each other either $p_{I}$ varies $(h=$ constant $)$ or $h$ varies $\left(p_{I}=\right.$ constant $)$. Assume further that 4.4 is satisfied. Then the critical points on the equilibrium paths can be classified as follows:

- the critical point is a limit point if

$$
\dot{p}_{\mathrm{I}}^{\mathrm{cr}}=0, \dot{h}=0 \quad \text { or } \quad \dot{h}^{\mathrm{cr}}=0, \dot{p}_{\mathrm{I}}=0,
$$

- the critical point is a branching point if

$$
\mathbf{v}_{i}^{\mathrm{T}} \mathbf{g}_{\mathrm{I} i}^{\mathrm{cr}}=0, \dot{h}=0 \quad \text { or } \quad \mathbf{v}_{i}^{\mathrm{T}}\left(\frac{\partial \mathbf{A}_{i}}{\partial h}\right)^{\mathrm{cr}}=0, \dot{p}_{\mathrm{I}}=0 .
$$


4.2. Neighborhood of critical points. Assume that the critical equilibrium state is known, i.e., it is known what the problem parameters are for the critical equilibrium state. It is a fundamental assumption for investigating the neighborhood of the critical points that under the condition $h=$ constant either $p_{\mathrm{I}}=$ constant and $p_{\mathrm{II}}$ varies or $p_{\mathrm{II}}=$ constant and $p_{\mathrm{I}}$ varies.

4.2.1. Branching point. Let $s$ [the arc length of the branching (or secondary) equilibrium path: $\tau=s-s_{0}$ ] be the governing parameter on the branching equilibrium path. Further let $p_{\mathrm{I}}\left(\tau=p_{\mathrm{I}}-p_{\mathrm{I}}^{c r}\right)$ be the governing parameter on the primary equilibrium path. Let us approach the neighborhood of the branching point on the equilibrium paths assuming that $p_{\text {II }}=$ constant, $h=$ constant both on the primary equilibrium path and on the branching equilibrium path.

According to relations 3.41 and 3.42 :

$$
\begin{gathered}
\text { if }()^{*}=\frac{\partial()}{\partial s}, \quad \mathbf{L}_{i k j}=2 \mathbf{J}_{i k j}+\mathbf{H}_{i k j}-\mathbf{M}_{\mathrm{I} i k j} p_{\mathrm{I}}, \\
\mathbf{K}_{i j} \dot{\mathbf{t}}_{j}-\mathbf{g}_{\mathrm{I} i} \dot{p}_{\mathrm{I}}=0, \quad \mathbf{K}_{i j} \ddot{\mathbf{t}}_{j}-\mathbf{g}_{\mathrm{I} i} \ddot{p}_{\mathrm{I}}=-\mathbf{L}_{i k j} \dot{\mathbf{t}}_{k} \dot{\mathbf{t}}_{j}+2 \mathbf{K}_{\mathrm{I} i j}^{\mathrm{C}} \dot{\mathbf{t}}_{j} \dot{p}_{\mathrm{I}},
\end{gathered}
$$

and

$$
\begin{gathered}
\text { if }()^{\nabla}=\frac{\partial()}{\partial p_{\mathrm{I}}}, \quad p_{\mathrm{I}}^{\nabla}=1, \\
\mathbf{K}_{i j} \mathbf{t}_{j}^{\nabla}-\mathbf{g}_{\mathrm{I} i}=0, \quad \mathbf{K}_{i j} \mathbf{t}_{j}^{\nabla \nabla}=-\mathbf{L}_{i k j} \mathbf{t}_{k}^{\nabla} \mathbf{t}_{j}^{\nabla}+2 \mathbf{K}_{\mathrm{I} i j}^{\mathrm{C}} \mathbf{t}_{j}^{\nabla}
\end{gathered}
$$

are the equilibrium equations on the branching and primary equilibrium paths, respectively.

Consider equations 4.12 and 4.13 at the branching point and let

$$
\dot{\mathbf{t}}_{j}^{\mathrm{cr}}=\xi_{0} \mathbf{t}_{j}^{\nabla \mathrm{cr}}+\xi_{1} \mathbf{u}_{j}, \quad \xi_{0}=\dot{p}_{\mathrm{I}}^{\mathrm{cr}}
$$

on the branching equilibrium path. Substituting into $4.12 p_{1}$ and taking 4.13$)_{1}$ into account we have

$$
\xi_{1} \mathbf{K}_{i j}^{\mathrm{cr}} \mathbf{u}_{j}=0
$$

which means that we get back $(4.3)_{1}$ at the branching point.

Let us multiply equilibrium equation $4.2{ }_{2}$ by $\mathbf{v}_{i}$ :

$$
\mathbf{v}_{i}^{\mathrm{T}} \mathbf{K}_{i j}^{\mathrm{cr}} \ddot{\mathbf{t}}_{j}^{\mathrm{cr}}-\mathbf{v}_{i}^{\mathrm{T}} \mathbf{g}_{\mathrm{I} i}^{\mathrm{cr}} \ddot{p}_{\mathrm{I}}^{\mathrm{cr}}=-\mathbf{v}_{i}^{\mathrm{T}} \mathbf{L}_{i k j}^{\mathrm{cr}} \dot{\mathbf{t}}_{k}^{\mathrm{cr}} \dot{\mathbf{t}}_{j}^{\mathrm{cr}}+2 \mathbf{v}_{i}^{\mathrm{T}} \mathbf{K}_{\mathrm{I} i j}^{\mathrm{C} c r} 1 \dot{p}_{\mathrm{I}}^{\mathrm{cr}} \dot{\mathbf{t}}_{j}^{\mathrm{cr}} .
$$

Utilizing 4.3 2 and (4.6) and substituting then (4.14) we arrive at the following equation:

$$
0=-\left[\mathbf{v}_{i}^{\mathrm{T}} \mathbf{L}_{i k j}^{\mathrm{cr}}\left(\xi_{0} \mathbf{t}_{k}^{\nabla \mathrm{cr}}+\xi_{1} \mathbf{u}_{k}\right)\left(\xi_{0} \mathbf{t}_{j}^{\nabla \mathrm{cr}}+\xi_{1} \mathbf{u}_{j}\right)-2 \mathbf{v}_{i}^{\mathrm{T}} \mathbf{K}_{\mathrm{I} i j}^{\mathrm{C} \mathrm{cr}}\left(\xi_{0} \mathbf{t}_{j}^{\nabla \mathrm{kr}}+\xi_{1} \mathbf{u}_{j}\right) \xi_{0}\right],
$$

or

$$
a_{\mathrm{B}} \xi_{1}^{2}+2 b_{\mathrm{B}} \xi_{1} \xi_{0}+c_{\mathrm{B}} \xi_{0}^{2}=0
$$

where

$$
\begin{gathered}
a_{\mathrm{B}}=\mathbf{v}_{i}^{\mathrm{T}} \mathbf{L}_{i k j}^{\mathrm{cr}} \mathbf{u}_{k} \mathbf{u}_{j}, \\
b_{\mathrm{B}}=\mathbf{v}_{i}^{\mathrm{T}} \mathbf{L}_{i k j}^{\mathrm{cr}} \mathbf{t}_{k}^{\nabla \mathrm{cr}} \mathbf{u}_{j}-\mathbf{v}_{i}^{\mathrm{T}} \mathbf{K}_{\mathrm{I} i j}^{\mathrm{Ccr}} \mathbf{u}_{j}, \\
c_{\mathrm{B}}=\mathbf{v}_{i}^{\mathrm{T}} \mathbf{L}_{i k j}^{\mathrm{cr}} \mathbf{t}_{k}^{\nabla \mathrm{cr}} \mathbf{t}_{j}^{\nabla \mathrm{cr}}-2 \mathbf{v}_{i}^{\mathrm{T}} \mathbf{K}_{\mathrm{I} i j}^{\mathrm{Ccr}} \mathbf{t}_{j}^{\nabla \mathrm{cr}} .
\end{gathered}
$$


With the normalization condition $2 \xi_{0}^{2}+\xi_{1}^{2}=1$ equation 4.17 yields $\xi_{1}$. The coefficients in equation 4.18 make possible a further classification of the branching point. If

$\left\{\begin{array}{l}a_{\mathrm{B}}=0, b_{\mathrm{B}} \neq 0 \\ a_{\mathrm{B}} \neq 0, b_{\mathrm{B}}^{2}-a_{\mathrm{B}} c_{\mathrm{B}}>0 \\ b_{\mathrm{B}}^{2}-a_{\mathrm{B}} c_{\mathrm{B}}<0 \\ b_{\mathrm{B}}^{2}-a_{\mathrm{B}} c_{\mathrm{B}}=0\end{array}\right\}$ the point is a simple $\left\{\begin{array}{l}\text { pitchfork bifurcation point, } \\ \text { transcritical bifurcation point } \\ \text { isola formating point, } \\ \text { cusp point. }\end{array}\right\}$

After multiplying relation $(4.13)_{2}$ by $\mathbf{v}_{i}$ and taking then $(4.3)_{2}$ into account we have

$$
0=-\mathbf{v}_{i}^{\mathrm{T}} \mathbf{L}_{i k j} \mathbf{t}_{k}^{\nabla} \mathbf{t}_{j}^{\nabla}+2 \mathbf{v}_{i}^{\mathrm{T}} \mathbf{K}_{\mathrm{I} i j}^{\mathrm{C}} \mathbf{t}_{j}^{\nabla}
$$

If $\xi_{1}$ is set to 1 a comparison of 4.16) and 4.21) yields

$$
\xi_{0}=\dot{p}_{\mathrm{I}}^{\mathrm{cr}}=-\frac{\mathbf{v}_{i}^{\mathrm{T}} \mathbf{L}_{i k j} \mathbf{u}_{k} \mathbf{u}_{j}}{2\left(\mathbf{v}_{i}^{\mathrm{T}} \mathbf{L}_{i k j}^{\mathrm{cr}} \mathbf{t}_{k}^{\nabla \mathrm{cr}} \mathbf{u}_{j}-\mathbf{v}_{i}^{\mathrm{T}} \mathbf{K}_{\mathrm{I} i j}^{\mathrm{C} c r} \mathbf{u}_{j}\right)} .
$$

For a symmetric branching it holds that

$$
\mathbf{v}_{i}^{\mathrm{T}} \mathbf{L}_{i k j}^{\mathrm{cr}} \mathbf{u}_{k} \mathbf{u}_{j}=0 \quad \text { hence } \quad \dot{p}_{\mathrm{I}}^{\mathrm{cr}}=0
$$

Let us supplement our investigations by the equilibrium equations at the branching point, which are presented here on the basis of the later equations (5.35) and (5.40).

On the bifurcation equilibrium path

$$
\begin{aligned}
\mathbf{K}_{i j}^{\mathrm{cr}} \dddot{\mathbf{t}}_{j}^{\mathrm{cr}}-\mathbf{g}_{\mathrm{I} i}^{\mathrm{cr}} \dddot{p}_{\mathrm{I}}^{\mathrm{cr}}+3 \mathbf{L}_{i k j}^{\mathrm{cr}} \dot{\mathbf{t}}_{k}^{\mathrm{cr}} \ddot{\mathbf{t}}_{j}^{\mathrm{cr}}-3 \mathbf{K}_{\mathrm{I} i j}^{\mathrm{Cr}} \ddot{\mathbf{t}}_{j}^{\mathrm{cr}} \dot{p}_{\mathrm{I}}^{\mathrm{cr}}- \\
-3 \mathbf{K}_{\mathrm{I} i j}^{\mathrm{Ccr}} \dot{\mathbf{t}}_{j}^{\mathrm{cr}} \ddot{p}_{\mathrm{I}}^{\mathrm{cr}}-3 \mathbf{M}_{\mathrm{I} i k j}^{\mathrm{cr}} \dot{\mathbf{t}}_{k}^{\mathrm{cr}} \dot{\mathbf{t}}_{j}^{\mathrm{cr}} \dot{p}_{\mathrm{I}}^{\mathrm{cr}}+\mathbf{N}_{i l k j}^{\mathrm{cr}} \dot{\mathbf{t}}_{l}^{\mathrm{cr}} \dot{\mathbf{t}}_{k}^{\mathrm{cr}} \dot{\mathbf{t}}_{j}^{\mathrm{cr}}=0,
\end{aligned}
$$

whereas on the primary equilibrium path

$$
\begin{aligned}
\mathbf{K}_{i j}^{\mathrm{cr}} \mathbf{t}_{j}^{\nabla \nabla \nabla \mathrm{cr}}+3 \mathbf{L}_{i k j}^{\mathrm{cr}} \mathbf{t}_{k}^{\nabla \mathrm{cr}} \mathbf{t}_{j}^{\nabla \nabla \mathrm{cr}}- & 3 \mathbf{K}_{\mathrm{I} i j}^{\mathrm{C} \mathrm{cr}} \mathbf{t}_{j}^{\nabla \nabla \mathrm{cr}}- \\
& -3 \mathbf{M}_{\mathrm{I} i k j}^{\mathrm{cr}} \mathbf{t}_{k}^{\nabla \mathrm{cr}} \mathbf{t}_{j}^{\nabla \mathrm{cr}}+\mathbf{N}_{i l k j}^{\mathrm{cr}} \mathbf{t}_{l}^{\nabla \mathrm{cr}} \mathbf{t}_{k}^{\nabla \mathrm{cr}} \mathbf{t}_{j}^{\nabla \mathrm{cr}}=0 .
\end{aligned}
$$

are the equilibrium equations.

On the basis of 4.14 $\left(\xi_{1}=1\right)$

$$
\ddot{\mathbf{t}}_{j}=\frac{\partial^{2} \mathbf{t}_{j}}{\partial p_{\mathrm{I}}^{2}}\left(\frac{\partial p_{\mathrm{I}}}{\partial s}\right)^{2}+\frac{\partial \mathbf{t}_{j}}{\partial p_{\mathrm{I}}} \frac{\partial^{2} p_{\mathrm{I}}}{\partial s^{2}}+\frac{\partial \mathbf{u}_{j}}{\partial s},
$$

which means that

at the branching point

$$
\ddot{\mathbf{t}}_{j}^{\mathrm{cr}}=\mathbf{t}_{j}^{\nabla \nabla \mathrm{cr}} \dot{p}_{\mathrm{I}}^{2 \mathrm{cr}}+\mathbf{t}_{j}^{\nabla \mathrm{cr}} \ddot{p}_{\mathrm{I}}^{\mathrm{cr}}+\dot{\mathbf{u}}_{j}
$$

Let us substitute 4.26 and 4.14 into 4.12, 2 taken at the branching point:

$$
\begin{aligned}
\mathbf{K}_{i j}^{\mathrm{cr}}\left(\mathbf{t}_{j}^{\nabla \nabla \mathrm{cr}} \dot{p}_{\mathrm{I}}^{2 \mathrm{cr}}+\mathbf{t}_{j}^{\nabla \mathrm{cr}} \ddot{p}_{\mathrm{I}}^{\mathrm{cr}}+\dot{\mathbf{u}}_{j}\right)-\mathbf{g}_{\mathrm{I} i}^{\mathrm{cr}} \ddot{p}_{\mathrm{I}}+ \\
\quad+\mathbf{L}_{i k j}^{\mathrm{cr}}\left(\mathbf{t}_{k}^{\nabla \mathrm{cr}} \dot{p}_{\mathrm{I}}^{\mathrm{cr}}+\mathbf{u}_{k}\right)\left(\mathbf{t}_{j}^{\nabla \mathrm{cr}} \dot{p}_{\mathrm{I}}^{\mathrm{cr}}+\mathbf{u}_{j}\right)-2 \mathbf{K}_{\mathrm{I} i j}^{\mathrm{Ccr}}\left(\mathbf{t}_{j}^{\nabla \mathrm{cr}} \dot{p}_{\mathrm{I}}^{\mathrm{cr}}+\mathbf{u}_{j}\right) \dot{p}_{\mathrm{I}}^{\mathrm{cr}}=0 .
\end{aligned}
$$

By utilizing equations 4.13) it follows from here that

$$
\mathbf{K}_{i j}^{\mathrm{cr}} \dot{\mathbf{u}}_{j}+2 \mathbf{L}_{i k j}^{\mathrm{cr}} \mathbf{t}_{k}^{\nabla \mathrm{cr}} \mathbf{u}_{j} \dot{p}_{\mathrm{I}}^{\mathrm{cr}}+\mathbf{L}_{i k j}^{\mathrm{cr}} \mathbf{u}_{k} \mathbf{u}_{j}-2 \mathbf{K}_{\mathrm{I} i j}^{\mathrm{Ccr}} \mathbf{u}_{j} \dot{p}_{\mathrm{I}}^{\mathrm{cr}}=0 .
$$


Multiply now this equation by $\mathbf{v}_{i}$. In view to equation 4.15 we get

$$
2 \mathbf{v}_{i}^{\mathrm{T}} \mathbf{L}_{i k j}^{\mathrm{cr}} \mathbf{t}_{j}^{\nabla \mathrm{cr}} \mathbf{u}_{j} \dot{p}_{\mathrm{I}}^{\mathrm{cr}}+\mathbf{v}_{i}^{\mathrm{T}} \mathbf{L}_{i k j}^{\mathrm{cr}} \mathbf{u}_{k} \mathbf{u}_{j}-2 \mathbf{v}_{i}^{\mathrm{T}} \mathbf{K}_{\mathrm{I} i j}^{\mathrm{Ccr}} \mathbf{u}_{j} \dot{p}_{\mathrm{I}}^{\mathrm{cr}}=0 .
$$

We can find 4.22 from 4.28 and $\dot{\mathbf{u}}_{j}$ from 4.27

Let us multiply equation (4.24) by $\mathbf{v}_{i}$ and substitute then relations (4.14) and 4.26. We have

$$
\begin{aligned}
& 3 \mathbf{v}_{i}^{\mathrm{T}} \mathbf{L}_{i k j}^{\mathrm{cr}}\left(\mathbf{t}_{k}^{\nabla \mathrm{cr}} \dot{p}_{\mathrm{I}}^{\mathrm{cr}}+\mathbf{u}_{k}\right)\left(\mathbf{t}_{j}^{\nabla \nabla \mathrm{cr}} \dot{p}_{\mathrm{I}}^{2 \mathrm{cr}}+\mathbf{t}_{j}^{\nabla \mathrm{cr}} \ddot{p}_{\mathrm{I}}^{\mathrm{cr}}+\dot{\mathbf{u}}_{j}\right)- \\
& -3 \mathbf{v}_{i}^{\mathrm{T}} \mathbf{K}_{\mathrm{I} i j}^{\mathrm{ccr}}\left(\mathbf{t}_{j}^{\nabla \nabla \mathrm{cr}} \dot{p}_{\mathrm{I}}^{2 \mathrm{cr}}+\mathbf{t}_{j}^{\nabla \mathrm{cr}} \ddot{p}_{\mathrm{I}}^{\mathrm{cr}}+\dot{\mathbf{u}}_{j}\right) \dot{p}_{\mathrm{I}}^{\mathrm{cr}}-3 \mathbf{v}_{i}^{\mathrm{T}} \mathbf{K}_{\mathrm{I} i j}^{\mathrm{Ccr}}\left(\mathbf{t}_{j}^{\nabla \mathrm{cr}} \dot{p}_{\mathrm{I}}^{\mathrm{cr}}+\mathbf{u}_{j}\right) \ddot{p}_{\mathrm{I}}^{\mathrm{cr}}- \\
& -3 \mathbf{v}_{i}^{\mathrm{T}} \mathbf{M}_{\mathrm{I} i k j}^{\mathrm{cr}}\left(\mathbf{t}_{j}^{\nabla c \mathrm{cr}} \dot{p}_{\mathrm{I}}^{\mathrm{cr}}+\mathbf{u}_{k}\right)\left(\mathbf{t}_{j}^{\nabla \mathrm{cr}} \dot{p}_{\mathrm{I}}^{\mathrm{cr}}+\mathbf{u}_{j}\right) \dot{p}_{\mathrm{I}}^{\mathrm{cr}}+ \\
& \quad+\mathbf{v}_{i}^{\mathrm{T}} \mathbf{N}_{i l k j}^{\mathrm{cr}}\left(\mathbf{t}_{l}^{\nabla \mathrm{cr}} \dot{p}_{\mathrm{I}}^{\mathrm{cr}}+\mathbf{u}_{l}\right)\left(\mathbf{t}_{k}^{\nabla \mathrm{cr}} \dot{p}_{\mathrm{I}}^{\mathrm{cr}}+\mathbf{u}_{k}\right)\left(\mathbf{t}_{j}^{\nabla \mathrm{cr}} \dot{p}_{\mathrm{I}}^{\mathrm{cr}}+\mathbf{u}_{j}\right)=0
\end{aligned}
$$

In view of 4.21) and equation

$$
\begin{aligned}
\mathbf{v}_{i}^{\mathrm{T}} 3 \mathbf{L}_{i k j}^{\mathrm{cr}} \mathbf{t}_{k}^{\nabla \mathrm{cr}} \mathbf{t}_{j}^{\nabla \nabla \mathrm{cr}}- & 3 \mathbf{v}_{i}^{\mathrm{T}} \mathbf{K}_{\mathrm{I} i j}^{\mathrm{C} \mathrm{cr}} \mathbf{t}_{j}^{\nabla \nabla \mathrm{cr}}- \\
& -3 \mathbf{v}_{i}^{\mathrm{T}} \mathbf{M}_{\mathrm{I} i k j}^{\mathrm{cr}} \mathbf{t}_{k}^{\nabla \mathrm{cr}} \mathbf{t}_{j}^{\nabla \mathrm{cr}}+\mathbf{v}_{i}^{\mathrm{T}} \mathbf{N}_{i l k j}^{\mathrm{cr}} \mathbf{t}_{l}^{\nabla \mathrm{cr}} \mathbf{t}_{k}^{\nabla \mathrm{cr}} \mathbf{t}_{j}^{\nabla \mathrm{cr}}=0
\end{aligned}
$$

obtained from 4.25 by multiplying it with $\mathbf{v}_{i}$ equation 4.29 yields

$$
\ddot{p}_{\mathrm{I}}^{\mathrm{cr}}=-\frac{A+B \dot{p}_{\mathrm{I}}^{\mathrm{cr}}+C \dot{p}_{\mathrm{I}}^{\mathrm{cr}}}{3\left(\mathbf{v}_{i}^{\mathrm{T}} \mathbf{L}_{i k j}^{\mathrm{cr}} \mathbf{t}_{k}^{\nabla \mathrm{cr}} \mathbf{u}_{j}-\mathbf{v}_{i}^{\mathrm{T}} \mathbf{K}_{\mathrm{I} i j}^{\mathrm{C} c r} \mathbf{u}_{j}\right)},
$$

where

$$
\begin{gathered}
A=\mathbf{v}_{i}^{\mathrm{T}} \mathbf{N}_{i l k j}^{\mathrm{cr}} \mathbf{u}_{l} \mathbf{u}_{k} \mathbf{u}_{j}+3 \mathbf{v}_{i}^{\mathrm{T}} \mathbf{L}_{i k j}^{\mathrm{cr}} \mathbf{u}_{k} \dot{\mathbf{u}}_{j} \\
B=3 \mathbf{v}_{i}^{\mathrm{T}} \mathbf{N}_{i l k j}^{\mathrm{cr}} \mathbf{t}_{l}^{\nabla \mathrm{cr}} \mathbf{u}_{k} \mathbf{u}_{j}+3 \mathbf{v}_{i}^{\mathrm{T}} \mathbf{L}_{i k j}^{\mathrm{cr}} \mathbf{t}_{k}^{\nabla \mathrm{cr}} \mathbf{u}_{k} \dot{\mathbf{u}}_{j}-3 \mathbf{v}_{i}^{\mathrm{T}} \mathbf{M}_{\mathrm{I} i k j}^{\mathrm{cr}} \mathbf{u}_{k} \mathbf{u}_{j}-3 \mathbf{v}_{i}^{\mathrm{T}} \mathbf{K}_{\mathrm{I} i j}^{\mathrm{Cr}} \dot{\mathbf{u}}_{j}, \\
C=\mathbf{v}_{i} \mathbf{N}_{i l k j}^{\mathrm{cr}} \mathbf{w}_{l}^{\mathrm{cr}} \mathbf{w}_{k}^{\mathrm{cr}} \mathbf{u}_{j}+3 \mathbf{v}_{i} \mathbf{L}_{i k j}^{\mathrm{cr}} \mathbf{u}_{k} \dot{\mathbf{w}}_{j}^{\mathrm{cr}}-3 \mathbf{v}_{i} \mathbf{M}_{\mathrm{I} i k j}^{\mathrm{cr}} \mathbf{w}_{k}^{\mathrm{cr}} \mathbf{u}_{j} .
\end{gathered}
$$

For a symmetric branching point $p_{\mathrm{I}}^{\mathrm{cr}}=0$ from 4.23 . Then 4.30 is simplified into

$$
\ddot{p}_{\mathrm{I}}^{\mathrm{cr}}=-\frac{A}{3\left(\mathbf{v}_{i}^{\mathrm{T}} \mathbf{L}_{i k j}^{\mathrm{cr}} \mathbf{t}_{k}^{\nabla \mathrm{cr}} \mathbf{u}_{j}-\mathbf{v}_{i}^{\mathrm{T}} \mathbf{K}_{\mathrm{I} i j}^{\mathrm{C} c r} \mathbf{u}_{j}\right)} .
$$

Since $\mathbf{K}_{i j}^{\mathrm{cr}}$ is singular in equations 4.12 4.13 which are taken at the branching point further equations are needed to ensure single valued solutions. Equation $4.12{ }_{1}$ should be supplemented by

$$
\mathbf{v}_{i}^{\mathrm{T}} \mathbf{L}_{i k j} \dot{\mathbf{t}}_{k} \dot{\mathbf{t}}_{j}-2 \mathbf{v}_{i}^{\mathrm{T}} \mathbf{K}_{\mathrm{I} i j}^{\mathrm{C}} \dot{\mathbf{t}}_{j} \dot{p}_{\mathrm{I}}=0
$$

equation $4.12{ }_{2}$ by

$$
\begin{aligned}
3 \mathbf{v}_{i}^{\mathrm{T}} \mathbf{L}_{i k j}^{\mathrm{cr}} \dot{\mathrm{t}}_{k}^{\mathrm{cr}} \ddot{\mathbf{t}}_{j}^{\mathrm{cr}}-3 \mathbf{v}_{i}^{\mathrm{T}} \mathbf{K}_{\mathrm{I} i j}^{\mathrm{C} c \mathrm{c}} \ddot{\mathbf{t}}_{j}^{\mathrm{cr}} \dot{p}_{\mathrm{I}}^{\mathrm{cr}}-3 \mathbf{v}_{i}^{\mathrm{T}} \mathbf{K}_{\mathrm{I} i j}^{\mathrm{C} c r} \dot{\mathrm{t}}_{j}^{\mathrm{cr}} \ddot{p}_{\mathrm{I}}^{\mathrm{cr}}- \\
-3 \mathbf{v}_{i}^{\mathrm{T}} \mathbf{M}_{\mathrm{I} i k j}^{\mathrm{cr}} \dot{\mathrm{t}}_{k}^{\mathrm{cr}} \dot{\mathrm{t}}_{j}^{\mathrm{cr}} \dot{p}_{\mathrm{I}}^{\mathrm{cr}}+\mathbf{v}_{i}^{\mathrm{T}} \mathbf{N}_{i l k j}^{\mathrm{cr}} \dot{\mathrm{t}}_{l}^{\mathrm{cr}} \dot{\mathrm{t}}_{k}^{\mathrm{cr}} \dot{\mathrm{t}}_{j}^{\mathrm{cr}}=0,
\end{aligned}
$$

equation 4.13 by

and finally equation $4.13{ }_{2}$ by

$$
\mathbf{v}_{i}^{\mathrm{T}} \mathbf{L}_{i k j} \mathbf{t}_{k}^{\nabla} \mathbf{t}_{j}^{\nabla}-2 \mathbf{v}_{i}^{\mathrm{T}} \mathbf{K}_{\mathrm{I} i j}^{\mathrm{C}} \mathbf{t}_{j}^{\nabla}=0
$$

$$
\mathbf{v}_{i}^{\mathrm{T}} 3 \mathbf{L}_{i k j}^{\mathrm{cr}} \mathbf{t}_{k}^{\nabla \mathrm{cr}} \mathbf{t}_{j}^{\nabla \nabla \mathrm{cr}}-3 \mathbf{v}_{i}^{\mathrm{T}} \mathbf{K}_{\mathrm{I} i j}^{\mathrm{Ccr}} \mathbf{t}_{j}^{\nabla \nabla \mathrm{cr}}-3 \mathbf{v}_{i}^{\mathrm{T}} \mathbf{M}_{\mathrm{I} i k j}^{\mathrm{cr}} \mathbf{t}_{k}^{\nabla \mathrm{cr}} \mathbf{t}_{j}^{\nabla \mathrm{cr}}+
$$




$$
+\mathbf{v}_{i}^{\mathrm{T}} \mathbf{N}_{i l k j}^{\mathrm{cr}} \mathbf{t}_{l}^{\nabla \mathrm{cr}} \mathbf{t}_{k}^{\nabla \mathrm{cr}} \mathbf{t}_{j}^{\nabla \mathrm{cr}}=0 .
$$

4.2.2. Limit point. At the limit point $\dot{p}_{\mathrm{I}}^{\mathrm{cr}}=0$. Hence

$$
\begin{gathered}
\dot{\mathbf{t}}_{j}^{\mathrm{cr}}=\xi_{1} \mathbf{u}_{j}, \quad \xi_{0}=\dot{p}_{\mathrm{I}}^{\mathrm{cr}}=0, \\
0=-\left[\mathbf{v}_{i}^{\mathrm{T}} \mathbf{L}_{i k j}^{\mathrm{cr}}\left(\xi_{1} \mathbf{u}_{k}\right)\left(\xi_{1} \mathbf{u}_{j}\right)-\mathbf{v}_{i}^{\mathrm{T}} \mathbf{g}_{\mathrm{I} i} \ddot{p}_{\mathrm{I}}^{\mathrm{cr}}\right]
\end{gathered}
$$

and

$$
a_{\mathrm{L}} \xi_{1}^{2}+d_{\mathrm{L}} \ddot{p}_{\mathrm{I}}^{\mathrm{cr}}=0, \quad \xi_{1}=1
$$

in which

$$
a_{\mathrm{L}}=\mathbf{v}_{i}^{\mathrm{T}} \mathbf{L}_{i k j}^{\mathrm{cr}} \mathbf{u}_{k} \mathbf{u}_{j}, \quad d_{\mathrm{L}}=-\mathbf{v}_{i}^{\mathrm{T}} \mathbf{g}_{\mathrm{I} i}
$$

$\ddot{p}_{\mathrm{I}}^{\text {cr }}$ can be calculated from 4.41. If $\xi_{1}=1$ we have

$$
\ddot{p}_{\mathrm{I}}^{\mathrm{cr}}=\frac{\mathbf{v}_{i}^{\mathrm{T}} \mathbf{L}_{i k j}^{\mathrm{kr}} \mathbf{u}_{k} \mathbf{u}_{j}}{\mathbf{v}_{i}^{\mathrm{T}} \mathbf{g}_{\mathrm{I} i}} .
$$

The limit points can be classified. If

$\left\{\begin{array}{l}a_{\mathrm{L}} \neq 0 \\ a_{\mathrm{L}} \neq 0, \quad e_{\mathrm{L}}=\mathbf{v}_{i}^{\mathrm{T}} 1\left(\frac{\partial \mathbf{L}_{i k j}}{\partial \mathbf{t}_{l}}\right)^{\mathrm{cr}} \mathbf{u}_{l} \mathbf{u}_{k} \mathbf{u}_{j} \neq 0\end{array}\right\}$ the limit point is $\left\{\begin{array}{l}\text { simple quadratic one }, \\ \text { simple cubic one. }\end{array}\right\}$

REMARK 4.5: The role of the problem parameters $p_{\mathrm{I}}$ and $p_{\mathrm{II}}$ can be interchanged in Subsection 4.2

\section{Determination of The FOlD Line OF Limit POINTS}

5.1. A brief survey. According to Paragraph 3.2.3.4 in Sububsection 3.2.3 the fold line is the locus of the limit points on those equilibrium paths that belong to the different values of an imperfection parameter. In the case of the imperfection parameters introduced in the present paper we can speak about

- the fold line of the load imperfection parameter $p_{\mathrm{II}}$, (then the geometrical imperfection parameter $h$ is constant), or

- the fold line of the geometrical imperfection parameter $h$, (then the load imperfection parameter $p_{\text {II }}$ is constant).

In both cases $p_{\mathrm{I}}$ is the fundamental load and the equilibrium paths together with the fold lines are defined as plane curves in the coordinate system $\left(p_{\mathrm{I}}, t_{m}\right)$ (in the domain of the parameters investigated).

Referring to Paragraph 3.2.3.1 we may also say that the fold line is a space curve the projections of which are the plane curves mentioned above.

We may lay emphasis on the following two ways for determining the fold line:

- The first is the application of a total stability analysis when under the condition $h=$ constant we determine the equilibrium surfaces and the equilibrium paths as surface and coordinate plane intersections in the coordinate system $\left(p_{\mathrm{II}}, p_{\mathrm{I}}, t_{m}\right)$ [or under the condition $p_{\mathrm{II}}=$ constant in the coordinate system $\left.\left(h, p_{\mathrm{I}}, t_{m}\right)\right]$. Then by joining the limit points on the equilibrium paths we can determine the fold line. 
- The second is the direct determination of the fold line. Then by the use of a previously found limit point we determine the fold line as a space curve applying the asymptotic numerical method or the Newton-Raphson iteration algorithm under the condition $h=$ constant [or $p_{\text {II }}=$ constant].

Equilibrium surfaces can imagined by determining the curves $p_{\text {II }}$ (or $h$ ) over the lines $p_{\mathrm{I}}=$ constant and $t_{m}=$ constant numerically (by applying, for example, the Newton-Rapson iteration algorithm on the basis of Subsection 3.2 .2 or the asymptotic numerical method detailed in Subsection 3.2.4.

During the numerical investigations it is advisable to select an imperfection parameter (load or geometrical one) or a displacement parameter for governing the computations.

In the sequel we touch upon the determination of the fold line by the use of the direct method.

The equilibrium state $\left(\bar{B}^{\mathrm{cr}}\right)$, which is the point of departure for calculating the fold line, and its problem parameters $p_{\mathrm{I} 0}, p_{\mathrm{II}}, h_{0}$ are assumed to be known.

Let $\tau$ be the governing parameter for the fold line starting from the state $\left(\bar{B}^{\mathrm{cr}}\right)$.

On the basis of equations 3.22 and $(3.23)_{2}$ equilibrium of state $\left(\bar{B}^{\text {cr }}\right)$ can be expressed by equation

$$
\mathbf{A}_{i}=\mathbf{b}_{i}-\mathbf{g}_{\mathrm{I} i} p_{\mathrm{I}}-\mathbf{g}_{\mathrm{II} i} p_{\mathrm{II}}=0 .
$$

For the critical equilibrium state it holds, according to 4.3 and the previous equation, that

$$
\mathbf{K}_{i j} \mathbf{u}_{j}=0, \quad \mathbf{v}_{i}^{\mathrm{T}} \mathbf{K}_{i j}=0, \quad \operatorname{det} \mathbf{K}_{i j}=0
$$

where $\mathbf{v}_{i}$ and $\mathbf{u}_{j}$ are the left and right eigenvectors belonging to a simple zero eigenvalue. Equations $(5.2)$ are referred to as constraint equations. The single valuedness of $\mathbf{u}_{j}$ is ensured by the normalization condition

$$
\mathbf{u}_{j}^{\mathrm{T}} \mathbf{u}_{j}-1=0 \text {. }
$$

Here (and in the sequel) it is not denoted in equations (5.1)- 5.3 that the quantities they contain are all taken in the equilibrium state $\left(\bar{B}^{\mathrm{cr}}\right)$.

Equations (5.1)-(5.3) are satisfied at each point of the space fold line - the quantities in them are taken at the point considered.

Let the state $\left(\bar{B}^{\mathrm{cr}}+\Delta \bar{B}^{\mathrm{cr}}\right)$ on the fold line be in a small neighborhood of the state $\left(\bar{B}^{\mathrm{cr}}\right)$. It is assumed that state $\left(\bar{B}^{\mathrm{cr}}+\Delta \bar{B}^{\mathrm{cr}}\right)$ is determined by the value $\Delta \tau$ of the governing parameter.

For state $\left(\bar{B}^{\mathrm{cr}}+\Delta \bar{B}^{\mathrm{cr}}\right)$ equations 5 (5.1)- 5.3 assume the following forms:

$$
\begin{gathered}
\mathbf{A}_{i}+\Delta \mathbf{A}_{i}=\mathbf{b}_{i}+\Delta \mathbf{b}_{i}-\left(\mathbf{g}_{\mathrm{I} i}+\Delta \mathbf{g}_{\mathrm{I} i}\right)\left(p_{\mathrm{I}}+\Delta p_{\mathrm{I}}\right)-\left(\mathbf{g}_{\mathrm{II} i}+\Delta \mathbf{g}_{\mathrm{II} i}\right)\left(p_{\mathrm{II}}+\Delta p_{\mathrm{II}}\right)=0 \\
\left(\mathbf{K}_{i j}+\Delta \mathbf{K}_{i j}\right)\left(\mathbf{u}_{j}+\Delta \mathbf{u}_{j}\right)=0 \\
\left(\mathbf{u}_{j}^{\mathrm{T}}+\Delta \mathbf{u}_{j}^{\mathrm{T}}\right)\left(\mathbf{u}_{j}+\Delta \mathbf{u}_{j}\right)-1=0
\end{gathered}
$$

Solution of the system of nonlinear equations (5.4)-(5.6) can be sought either by the Newton-Raphson iteration algorithm or by the asymptotic numerical method. 
5.2. Fold line of the limit points for a load imperfection. On the limit point fold line of load imperfection $h=h_{0}=$ constant and $p_{\text {II }}$ is the imperfection parameter.

The governing parameter along the fold line can be either the load or a displacement component. In the first case $\tau=p_{\text {II }}-p_{\text {II } 0}$, in the second one $\tau=t_{m}-t_{m 0}$ is the governing parameter.

\subsubsection{Solution by the Newton-Rapson iteration algorithm.}

5.2.1.1. First, let us assume that the load is the governing parameter $\left(\tau=p_{\text {II }}-\right.$ $\left.p_{\text {IIO }}, \Delta \tau=\Delta p_{\text {II }}\right)$. Solution of the equation system (5.4)-(5.6) is sought by the series of the increments $\Delta \mathbf{t}_{\underline{s} j}, \Delta p_{\text {II }}, \Delta \mathbf{u}_{\underline{s} j} ; \underline{\mathbf{s}}=\underline{1}, \underline{2}, \cdots,\left(p_{\text {II }}=p_{\text {II } 0}, \Delta p_{\text {II }}=\right.$ constant $)$ :

$$
\begin{aligned}
\mathbf{t}_{j}+\Delta \mathbf{t}_{j} & =\mathbf{t}_{j}+\Delta \mathbf{t}_{\underline{1} j}+\Delta \mathbf{t}_{\underline{2} j}+\cdots+\Delta \mathbf{t}_{\underline{s} j}+\cdots, \\
p_{\mathrm{I}}+\Delta p_{\mathrm{I}} & =p_{\mathrm{I}}+\Delta p_{\underline{I} \underline{1}}+\Delta p_{\mathrm{I} \underline{2}}+\cdots+\Delta p_{\mathrm{Is}}+\cdots, \\
\mathbf{u}_{j}+\Delta \mathbf{u}_{j} & =\mathbf{u}_{j}+\Delta \mathbf{u}_{\underline{1} j}+\Delta \mathbf{u}_{\underline{2} j}+\cdots+\Delta \mathbf{u}_{\underline{\mathbf{s}} j}+\cdots
\end{aligned}
$$

The linearized forms of equations (5.4)-(5.6) $\left(h=h_{0}=\right.$ constant, $\left.\Delta h=0\right)$ are given by:

$$
\begin{gathered}
\mathbf{A}_{i}+\frac{\partial \mathbf{A}_{i}}{\partial \mathbf{t}_{j}} \Delta \mathbf{t}_{j}-\mathbf{g}_{\mathrm{I} i} \Delta p_{\mathrm{I}}-\mathbf{g}_{\mathrm{II} i} \Delta p_{\mathrm{II}}=0 \\
\mathbf{K}_{i j} \mathbf{u}_{j}+\frac{\partial \mathbf{K}_{i j}}{\partial \mathbf{t}_{k}} \Delta \mathbf{t}_{k} \mathbf{u}_{j}+\frac{\partial \mathbf{K}_{i j}}{\partial p_{\mathrm{I}}} \mathbf{u}_{j} \Delta p_{\mathrm{I}}+\frac{\partial \mathbf{K}_{i j}}{\partial p_{\mathrm{II}}} \mathbf{u}_{j} \Delta p_{\mathrm{II}}+\mathbf{K}_{i j} \Delta \mathbf{u}_{j}=0, \\
\mathbf{u}_{j}^{\mathrm{T}} \Delta \mathbf{u}_{j}=0 .
\end{gathered}
$$

Making use of relations 5.32 5.36 (5.37) 2 and 5.38 presented in the later Subsection 5.2 .2 , equations for the $\underline{s}^{\text {th }}$ iteration step can be gathered in a simple matrix form:

$$
\begin{aligned}
& {\left[\begin{array}{ccc}
\mathbf{K}_{\underline{\mathbf{s}} i j} & 0 & -\mathbf{g}_{\underline{I} \underline{\underline{s}} i} \\
\mathbf{L}_{\underline{\mathbf{s}} i k j} \mathbf{u}_{\underline{\mathbf{s}} k} & \mathbf{K}_{\underline{\mathbf{s}} i j} & -\mathbf{K}_{\underline{\mathrm{I}} i j}^{\mathrm{C}} \mathbf{u}_{\underline{\mathbf{s}} j} \\
0 & \mathbf{u}_{\underline{\mathbf{s}} j}^{\mathrm{T}} & 0
\end{array}\right]\left[\begin{array}{c}
\Delta \mathbf{t}_{\underline{\mathbf{s}} j} \\
\Delta \mathbf{u}_{\underline{\mathbf{s}} j} \\
\Delta p_{\underline{I \underline{s}}}
\end{array}\right]=} \\
& =-\left[\begin{array}{c}
\mathbf{b}_{\underline{\underline{s}} i}-\left[\mathbf{g}_{\mathrm{I} \underline{\underline{s}} i} p_{\mathrm{I} \underline{\underline{s}}}+\mathbf{g}_{\mathrm{II} \underline{s} i} p_{\mathrm{II}}\right] \\
\mathbf{K}_{\underline{\mathbf{s}} i j} \mathbf{u}_{\underline{\mathbf{s}} j} \\
\mathbf{u}_{\underline{\mathrm{s}} k}^{\mathrm{T}} \mathbf{u}_{\underline{\mathbf{s}} k}-1
\end{array}\right]-\left[\begin{array}{c}
-\mathbf{g}_{\mathrm{II} \underline{s} i} \\
-\mathbf{K}_{\mathrm{II}}^{\mathrm{C}} \mathbf{u}_{\underline{\mathrm{s}} j} \\
0
\end{array}\right] \Delta p_{\mathrm{II}} .
\end{aligned}
$$

If $\Delta p_{\text {II }}$ is given $(5.13)$ is a system of linear equations for calculating the unknowns $\Delta \mathbf{t}_{\underline{\mathbf{s}} j}, \Delta \mathbf{u}_{\underline{\mathrm{s}} j}$ and $\Delta p_{\mathrm{Is}}$. Introducing the notations $\mathbf{t}_{j}=\mathbf{t}_{\underline{1 j}}, \mathbf{u}_{j}=\mathbf{u}_{\underline{1 j}}, p_{\mathrm{I}}=p_{\mathrm{I} \underline{1}}$ the variables of the $\underline{s}^{\text {th }}$ step can be obtained form the relations

$$
\begin{aligned}
\mathbf{t}_{\underline{\mathrm{s}} j} & =\mathbf{t}_{\underline{1} j}+\Delta \mathbf{t}_{\underline{1 j}}+\Delta \mathbf{t}_{\underline{2} j}+\cdots+\Delta \mathbf{t}_{\underline{\mathrm{s}-1} j}, & & \Delta \mathbf{t}_{\underline{\mathrm{s}} j}, \\
\mathbf{u}_{\underline{\mathrm{s}} j} & =\mathbf{u}_{\underline{1} j}+\Delta \mathbf{u}_{\underline{1} j}+\Delta \mathbf{u}_{\underline{2} j}+\cdots+\Delta \mathbf{u}_{\underline{\mathrm{s}-1} j}, & & \Delta \mathbf{u}_{\underline{\mathbf{s}} j}, \\
p_{\mathrm{I} \underline{\mathrm{s}}} & =p_{\underline{\mathrm{I}} \underline{1}}+\Delta p_{\mathrm{I} \underline{1}}+\Delta p_{\mathrm{I} \underline{2}}+\cdots+\Delta p_{\mathrm{Is}-1}, & & \Delta p_{\mathrm{I} \underline{\mathrm{s}}}
\end{aligned}
$$


The right side of equation 5.13 tends to zero at the end of iteration. This means that $\Delta \mathbf{t}_{\mathrm{s} j}, \Delta \mathbf{u}_{\mathrm{s} j}, \Delta p_{\text {Is }} \rightarrow 0$ by satisfying the prescribed error limit. Thus in this manner the right side of equation (5.13) approximately coincides with equations (5.4)(5.6) which determine the state $\left(\overline{B^{\mathrm{cr}}}+\Delta \bar{B}^{\mathrm{cr}}\right)$. At the end of the iteration, that is to say, it holds that

$$
\begin{gathered}
\mathbf{b}_{\underline{\mathbf{s}} i}-\mathbf{g}_{\mathrm{I} \underline{s} i} p_{\mathrm{I} \underline{s}}-\mathbf{g}_{\mathrm{II} \underline{s} i}\left(p_{\mathrm{II}}+\Delta p_{\mathrm{II}}\right) \Longleftrightarrow \mathbf{A}_{i}+\Delta \mathbf{A}_{i} \\
\mathbf{K}_{\underline{\mathbf{s}} i j}=\mathbf{K}_{\underline{\mathbf{s}} i j}^{\mathrm{L}}+\mathbf{K}_{\underline{\mathbf{s}} i j}^{\mathrm{G}}-p_{\underline{I} \underline{\underline{I}}} \mathbf{K}_{\mathrm{I} \underline{s} i j}^{\mathrm{C}}-p_{\mathrm{II}} \mathbf{K}_{\mathrm{II} \underline{s} i j}^{\mathrm{C}}, \\
\left(\mathbf{K}_{\underline{\mathbf{s}} i j}-\Delta p_{\mathrm{II}} \mathbf{K}_{\mathrm{II} \underline{s} i j}^{\mathrm{C}}\right) \mathbf{u}_{\underline{\mathbf{s}} j}=\left[\mathbf{K}_{\underline{\mathbf{s}} i j}^{\mathrm{L}}+\mathbf{K}_{\underline{\mathbf{s}} i j}^{\mathrm{G}}-p_{\mathrm{I} \underline{\underline{s}}} \mathbf{K}_{\mathrm{I} \underline{\mathrm{s}} i j}^{\mathrm{C}}-\left(p_{\mathrm{II}}+\Delta p_{\mathrm{II}}\right) \mathbf{K}_{\mathrm{II} \underline{\mathbf{s}} i j}^{\mathrm{C}}\right] \mathbf{u}_{\underline{\mathbf{s}} j} \Longleftrightarrow \\
\Longleftrightarrow\left(\mathbf{K}_{i j}+\Delta \mathbf{K}_{i j}\right)\left(\mathbf{u}_{j}+\Delta \mathbf{u}_{j}\right), \\
\mathbf{u}_{\underline{\mathbf{s}} k}^{\mathrm{T}} \mathbf{u}_{\underline{\mathbf{s}} k}-1 \Longleftrightarrow(5.18)
\end{gathered}
$$

5.2.1.2. Second, let us assume that a displacement component is the governing parameter $\left(\tau=t_{m}-t_{m 0}, \Delta \tau=\Delta t_{m}\right)$. Solution of the equation system (5.4)-(5.6) is sought by the series of the increments $\Delta \mathbf{t}_{\underline{\mathrm{s}} \alpha}, \Delta p_{\mathrm{I} \underline{\mathrm{s}}}, \Delta p_{\mathrm{II} \underline{\underline{s}}}, \Delta \mathbf{u}_{\underline{\mathrm{s}} j} ; \underline{\mathbf{s}}=\underline{1}, \underline{2}, \ldots$; $\left(t_{m}=t_{m 0}=\right.$ constant, $\Delta t_{m}=$ constant $)$ :

$$
\begin{aligned}
\mathbf{t}_{\alpha}+\Delta \mathbf{t}_{\alpha}=\mathbf{t}_{\alpha}+\Delta \mathbf{t}_{\underline{1} \alpha}+\Delta \mathbf{t}_{\underline{2} \alpha}+\cdots+\Delta \mathbf{t}_{\underline{\mathrm{s}} \alpha}+\cdots, \quad \alpha=1,2, \cdots, m-1 \\
p_{\mathrm{I}}+\Delta p_{\mathrm{I}}=p_{\mathrm{I}}+\Delta p_{\mathrm{I} \underline{1}}+\Delta p_{\mathrm{I} \underline{2}}+\cdots+\Delta p_{\mathrm{II}}+\cdots, \\
p_{\mathrm{II}}+\Delta p_{\mathrm{II}}=p_{\mathrm{II}}+\Delta p_{\mathrm{II} \underline{1}}+\Delta p_{\mathrm{II} \underline{2}}+\cdots+\Delta p_{\mathrm{II} \underline{\underline{s}}}+\cdots, \\
\mathbf{u}_{j}+\Delta \mathbf{u}_{j}=\mathbf{u}_{j}+\Delta \mathbf{u}_{\underline{1} j}+\Delta \underline{\mathbf{u}}_{\underline{2} j}+\cdots+\Delta \mathbf{u}_{\underline{\mathrm{s}} j}+\cdots
\end{aligned}
$$

Here and in the sequel $\alpha, \beta=1,2, \cdots, m-1$, where $m$ is the degree of freedom (independently of the previous notational convention in matrix notation, $m$ may be the subscript of any displacement parameter; after selecting it, however, its value is fixed and can not take other values).

The linearized form of equations (5.4)-(5.6) is given by

$$
\begin{gathered}
\mathbf{A}_{i}+\frac{\partial \mathbf{A}_{i}}{\partial \mathbf{t}_{j}} \Delta \mathbf{t}_{j}+\frac{\partial \mathbf{A}_{i}}{\partial t_{m}} \Delta t_{m}-\mathbf{g}_{\mathrm{I} i} \Delta p_{\mathrm{I}}-\mathbf{g}_{\mathrm{II} i} \Delta p_{\mathrm{II}}=0 \\
\mathbf{K}_{i j} \mathbf{u}_{j}+\frac{\partial \mathbf{K}_{i j}}{\partial \mathbf{t}_{\beta}} \mathbf{u}_{j} \Delta \mathbf{t}_{\beta}+\frac{\partial \mathbf{K}_{i j}}{\partial t_{m}} \mathbf{u}_{j} \Delta t_{m}+\frac{\partial \mathbf{K}_{i j}}{\partial p_{\mathrm{I}}} \mathbf{u}_{j} \Delta p_{\mathrm{I}}+\frac{\partial \mathbf{K}_{1 j}}{\partial p_{\mathrm{II}}} \mathbf{u}_{j} \Delta p_{\mathrm{II}}+\mathbf{K}_{i j} \Delta \mathbf{u}_{j}=0 \\
\mathbf{u}_{j}^{\mathrm{T}} \Delta \mathbf{u}_{j}=0 .
\end{gathered}
$$

Equations for the $\underline{s}^{\text {th }}$ iteration step can now be gathered in the following simple matrix form:

$$
\left[\begin{array}{cccc}
\mathbf{K}_{\underline{\mathbf{s}} i \beta} & 0 & -\mathbf{g}_{\mathrm{I} \underline{\underline{s}} i} & -\mathbf{g}_{\mathrm{II} \underline{\underline{s}} i} \\
\mathbf{L}_{\underline{\underline{s}} i k \beta} \mathbf{u}_{\underline{\mathbf{s}} k} & \mathbf{K}_{\underline{\mathbf{s}} i j} & -\mathbf{K}_{\mathrm{I} \underline{\underline{s}} i k}^{\mathrm{C}} \mathbf{u}_{\underline{\mathbf{s}} k} & -\mathbf{K}_{\mathrm{II} \underline{s} i k}^{\mathrm{C}} \mathbf{u}_{\underline{\mathbf{s}} k} \\
0 & \mathbf{u}_{\underline{\mathbf{s}} j}^{\mathrm{T}} & 0 & 0
\end{array}\right]\left[\begin{array}{c}
\Delta \mathbf{t}_{\underline{\mathbf{s}} \beta} \\
\Delta \mathbf{u}_{\underline{\mathbf{s}} j} \\
\Delta p_{\mathrm{I} \underline{\underline{s}}} \\
\Delta p_{\mathrm{II} \underline{\underline{s}}}
\end{array}\right]=
$$




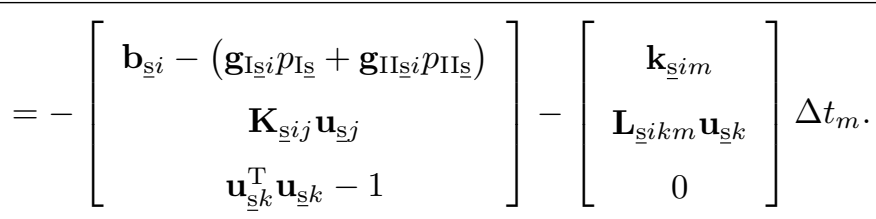

For a given $\Delta t_{m}$ equation (5.27) is a linear equation system for calculating the unknowns $\Delta \mathbf{t}_{\underline{\underline{s}} \beta}, \Delta \mathbf{u}_{\underline{\mathbf{s}} \beta}, \Delta \mathbf{u}_{\underline{\mathbf{s}} m}$ and $\Delta p_{\text {Is }}, \Delta p_{\text {II } \underline{\underline{s}}}$. Introducing the notations $\mathbf{t}_{j}=\mathbf{t}_{\underline{1} j}$, $\mathbf{u}_{j}=\mathbf{u}_{\underline{1} j}, p_{\mathrm{I}}=p_{\mathrm{I} \underline{1}}, p_{\mathrm{II}}=p_{\mathrm{II} \underline{1}}$ the variables of the $\underline{s}^{\text {th }}$ step can be obtained from the relations

$$
\begin{aligned}
& \mathbf{t}_{\underline{\mathbf{s}} \beta}=\mathbf{t}_{\underline{1} \beta}+\Delta \mathbf{t}_{\underline{1} \beta}+\Delta \mathbf{t}_{\underline{2} \beta}+\cdots+\Delta \mathbf{t}_{\underline{\mathbf{s}-1} \beta}, \quad \Delta \mathbf{t}_{\underline{\mathbf{s}} \beta}, \\
& \mathbf{u}_{\underline{\mathrm{s}} j}=\mathbf{u}_{\underline{1} j}+\Delta \mathbf{u}_{\underline{1} j}+\Delta \underline{\mathbf{u}}_{2 j}+\cdots+\Delta \underline{\mathbf{u}_{\mathbf{s}-1}}, \quad \Delta \mathbf{u}_{\underline{\mathbf{s}} j}, \\
& p_{\text {I } \underline{\mathrm{S}}}=p_{\mathrm{I} \underline{1}}+1 \Delta p_{\mathrm{I} \underline{1}}+\Delta p_{\mathrm{I} \underline{2}}+\cdots+\Delta p_{\text {Is }-1}, \quad \Delta p_{\text {I } \underline{\mathrm{I}}} \\
& p_{\mathrm{II} \underline{\mathrm{s}}}=p_{\mathrm{II} \underline{1}}+\Delta p_{\mathrm{II} \underline{1}}+\Delta p_{\mathrm{II} \underline{2}}+\cdots+\Delta p_{\mathrm{IIs}-1}, \quad \Delta p_{\mathrm{II} \underline{\mathrm{s}}} \text {. }
\end{aligned}
$$

The right side of equation (5.27) tends to zero at the end of iteration. This means that $\Delta \mathbf{t}_{\underline{\mathrm{s}} \beta}, \Delta \mathbf{u}_{\underline{\mathrm{s}} j}, \Delta p_{\mathrm{Is}}, \Delta p_{\mathrm{IIs}} \rightarrow 0$ by satisfying the prescribed error limit. The right and left sides of equation (5.27), which tend to zero, ensure together the fulfillment of equations (5.4) $-(5.6)$ that determine the state $\left(\bar{B}^{\mathrm{cr}}+\Delta \bar{B}^{\mathrm{cr}}\right)$ of the body.

5.2.2. Solution by using the asymptotic numerical method. For applying the asymptotic numerical method (ANM) it is worth gathering the corresponding equilibrium equations on the basis of subsection $3.2 .4\left(h=h_{0}=\right.$ constant, $\dot{h}=\ddot{h}=\cdots=0$ !):

$$
\begin{gathered}
\mathbf{A}_{i}=\mathbf{b}_{i}-\mathbf{g}_{\mathrm{I} i} p_{\mathrm{I}}-\mathbf{g}_{\mathrm{II} i} p_{\mathrm{II}}=0 \\
\dot{\mathbf{A}}_{i}=\mathbf{K}_{i j} \dot{\mathbf{t}}_{j}-\mathbf{g}_{\mathrm{I} i} \dot{p}_{\mathrm{I}}-\mathbf{g}_{\mathrm{II} i} \dot{p}_{\mathrm{II}}=0 \\
\ddot{\mathbf{A}}_{i}=\mathbf{K}_{i j} \ddot{\mathbf{t}}_{j}-\mathbf{g}_{\mathrm{I} i} \ddot{p}_{\mathrm{I}}-\mathbf{g}_{\mathrm{II} i} \ddot{p}_{\mathrm{II}}+\mathbf{L}_{i k j} \dot{\mathbf{t}}_{k} \dot{\mathbf{t}}_{j}-2 \mathbf{K}_{\mathrm{I} i j}^{\mathrm{C}} \dot{\mathbf{t}}_{j} \dot{p}_{\mathrm{I}}-2 \mathbf{K}_{\mathrm{II} i j}^{\mathrm{C}} \dot{\mathbf{t}}_{j} \dot{p}_{\mathrm{II}}=0 \\
\dddot{\mathbf{A}}_{i}=\mathbf{K}_{i j} \dddot{\mathbf{t}}_{j}-\mathbf{g}_{\mathrm{I} i} \dddot{p}_{\mathrm{I}}-\mathbf{g}_{\mathrm{II} i} \dddot{p}_{\mathrm{II}}+3 \mathbf{L}_{i k j} \dot{\mathbf{t}}_{k} \ddot{\mathbf{t}}_{j}-3 \mathbf{K}_{\mathrm{I} i j}^{\mathrm{C}} \ddot{\mathbf{t}}_{j} \dot{p}_{\mathrm{I}}-3 \mathbf{K}_{\mathrm{II} i j}^{\mathrm{C}} \ddot{\mathbf{t}}_{j} \dot{p}_{\mathrm{II}}- \\
-3 \mathbf{K}_{\mathrm{I} i j}^{\mathrm{C}} \dot{\mathbf{t}}_{j} \ddot{p}_{\mathrm{I}}-3 \mathbf{K}_{\mathrm{II} i j}^{\mathrm{C}} \dot{\mathbf{t}}_{j} \ddot{p}_{\mathrm{II}}+\mathbf{N}_{i l k j} \dot{\mathbf{t}}_{l} \dot{\mathbf{t}}_{k} \dot{\mathbf{t}}_{j}-3 \mathbf{M}_{\mathrm{I} i k j} \dot{\mathbf{t}}_{k} \dot{\mathbf{t}}_{j} \dot{p}_{\mathrm{I}}-3 \mathbf{M}_{\mathrm{II} i k j} \dot{\mathbf{t}}_{k} \dot{\mathbf{t}}_{j} \dot{p}_{\mathrm{II}}=0
\end{gathered}
$$

where

$$
\begin{gathered}
\mathbf{K}_{i j}=\frac{\partial \mathbf{A}_{i}}{\partial \mathbf{t}_{j}}=\mathbf{K}_{i j}^{\mathrm{L}}+\mathbf{K}_{i j}^{\mathrm{G}}-\mathbf{K}_{\mathrm{I} i j}^{\mathrm{C}} p_{\mathrm{I}}-\mathbf{K}_{\mathrm{II} i j}^{\mathrm{C}} p_{\mathrm{II}}, \\
\frac{\partial \mathbf{b}_{i}}{\partial \mathbf{t}_{j}}=\mathbf{K}_{i j}^{\mathrm{L}}+\mathbf{K}_{i j}^{\mathrm{G}}, \quad-\frac{\partial \mathbf{g}_{\mathrm{N} i}}{\partial \mathbf{t}_{j}}=-\mathbf{K}_{\mathrm{N} i j}^{\mathrm{C}}, \quad \mathrm{N}=\mathrm{I}, \mathrm{II}, \\
\mathbf{L}_{i k j}=\frac{\partial \mathbf{K}_{i j}}{\partial \mathbf{t}_{k}}=\frac{\partial}{\partial \mathbf{t}_{k}} \frac{\partial \mathbf{A}_{i}}{\partial \mathbf{t}_{j}}=2 \mathbf{J}_{i k j}+\mathbf{H}_{i k j}-\mathbf{M}_{\mathrm{I} i k j} p_{\mathrm{I}}-\mathbf{M}_{\mathrm{II} i k j} p_{\mathrm{II}}, \\
\frac{\partial\left(\mathbf{K}_{i j}^{\mathrm{L}}+\mathbf{K}_{i j}^{\mathrm{G}}\right)}{\partial \mathbf{t}_{k}}=2 \mathbf{J}_{i k j}+\mathbf{H}_{i k j}, \quad-\frac{\partial \mathbf{K}_{\mathrm{N} i j}^{\mathrm{C}}}{\partial \mathbf{t}_{k}}=-\mathbf{M}_{\mathrm{N} k i j}, \quad \mathrm{~N}=\mathrm{I}, \mathrm{II}, \\
\mathbf{N}_{i l k j}=\frac{\partial \mathbf{L}_{i k j}}{\partial \mathbf{t}_{l}}=\frac{\partial}{\partial \mathbf{t}_{l}} \frac{\partial}{\partial \mathbf{t}_{k}} \frac{\partial \mathbf{A}_{i}}{\partial \mathbf{t}_{j}}=\frac{\partial\left(2 \mathbf{J}_{i k j}+\mathbf{H}_{i k j}\right)}{\partial \mathbf{t}_{l}} .
\end{gathered}
$$


Note that equation 5.40 is a consequence of equation

$$
-\frac{\partial \mathbf{M}_{\mathrm{N} k i j}}{\partial \mathbf{t}_{l}}=0, \quad \mathrm{~N}=\mathrm{I}, \mathrm{II} ;
$$

for comparison see 3.35 and equation

$$
\delta \mathbf{t}_{i} \mathbf{N}_{i l k j} \dot{\mathbf{t}}_{l} \dot{\mathbf{t}}_{k} \dot{\mathbf{t}}_{j}=3 \int_{(B)} \delta \dot{u}_{m ; k} \dot{u}_{; l}^{m} C^{k l p q} \dot{u}_{; p}^{s} \dot{u}_{s ; q} \mathrm{~d} V
$$

which follows from relation (3.34).

Matrix $\mathbf{L}_{i k j}$ in equations (5.38) and (5.40) is symmetric with respect to the index pair $k j$. Because of its definition - see equation $\left(5.40\right.$ - matrix $\mathbf{N}_{i l k j}$ is also symmetric with respect to the indices $l, k, j$. As regards the further derivatives it is important to mention that

$$
\frac{\partial \mathbf{N}_{l i k j}}{\partial \mathbf{t}_{h}}=\frac{\partial}{\partial \mathbf{t}_{h}} \frac{\partial \mathbf{L}_{i k j}}{\partial \mathbf{t}_{l}}=0 .
$$

This result is a consequence of equation 5.42.

There will be a need for the derivatives of $(5.2)_{1}$ and $(5.3)$ :

$$
\begin{aligned}
\left(\mathbf{K}_{i j} \mathbf{u}_{j}\right)^{\cdot}=\left(\frac{\partial \mathbf{K}_{i j}}{\partial \mathbf{t}_{k}} \dot{\mathbf{t}}_{k}+\right. & \left.\frac{\partial \mathbf{K}_{i j}}{\partial p_{\mathrm{I}}} \dot{p}_{\mathrm{I}}+\frac{\partial \mathbf{K}_{i j}}{\partial p_{\mathrm{II}}} \dot{p}_{\mathrm{II}}\right) \mathbf{u}_{j}+\mathbf{K}_{i j} \dot{\mathbf{u}}_{j}= \\
& =\mathbf{L}_{i k j} \mathbf{u}_{j} \dot{\mathbf{t}}_{k}-\mathbf{K}_{\mathrm{I} i j}^{\mathrm{C}} \dot{p}_{\mathrm{I}} \mathbf{u}_{j}-\mathbf{K}_{\mathrm{II} i j}^{\mathrm{C}} \dot{p}_{\mathrm{II}} \mathbf{u}_{j}+\mathbf{K}_{i j} \dot{\mathbf{u}}_{j}=0,
\end{aligned}
$$

$$
\begin{gathered}
\left(\mathbf{K}_{i j} \mathbf{u}_{j}\right)^{*}=\mathbf{N}_{i l k j} \mathbf{u}_{j} \dot{\mathbf{t}}_{l} \dot{\mathbf{t}}_{k}-2 \mathbf{M}_{\mathrm{I} i k j} \dot{p}_{\mathrm{I}} \mathbf{u}_{j} \dot{\mathbf{t}}_{k}-2 \mathbf{M}_{\mathrm{II} i k j} \dot{p}_{\mathrm{II}} \mathbf{u}_{j} \dot{\mathbf{t}}_{k}+2 \mathbf{L}_{i k j} \dot{\mathbf{u}}_{j} \dot{\mathbf{t}}_{k}+\mathbf{L}_{i k j} \mathbf{u}_{j} \ddot{\mathbf{t}}_{k}- \\
-\mathbf{K}_{\mathrm{I} i j}^{\mathrm{C}} \ddot{p}_{\mathrm{I}} \mathbf{u}_{j}-\mathbf{K}_{\mathrm{II} i j}^{\mathrm{C}} \ddot{p}_{\mathrm{II}} \mathbf{u}_{j}-2 \mathbf{K}_{\mathrm{I} i j}^{\mathrm{C}} \dot{p}_{\mathrm{I}} \dot{\mathbf{u}}_{j}-2 \mathbf{K}_{\mathrm{II} i j}^{\mathrm{C}} \dot{p}_{\mathrm{II}} \dot{\mathbf{u}}_{j}+\mathbf{K}_{i j} \ddot{\mathbf{u}}_{j}=0 \\
\mathbf{u}_{j}^{\mathrm{T}} \dot{\mathbf{u}}_{j}=0 \\
\dot{\mathbf{u}}_{j}^{\mathrm{T}} \dot{\mathbf{u}}_{j}+\mathbf{u}_{j}^{\mathrm{T}} \ddot{\mathbf{u}}_{j}=0 .
\end{gathered}
$$

5.2.2.1. First, let us find the equilibrium state $\left(\bar{B}^{\mathrm{cr}}+\Delta \bar{B}^{\mathrm{cr}}\right)$ by using the load as governing parameter, i.e., in the same manner as before $\left(\tau=p_{\mathrm{II}}-p_{\mathrm{II} 0}, \Delta \tau=\Delta p_{\mathrm{II}}\right)$. It is obvious that

$$
\begin{aligned}
\mathbf{t}_{i}(\tau) & =\mathbf{t}_{i}+\dot{\mathbf{t}}_{i} \tau+\frac{1}{2} \ddot{\mathbf{t}}_{i} \tau^{2}+\frac{1}{6} \dddot{\mathbf{t}}_{i} \tau^{3}+\cdots, \\
p_{\mathrm{I}}(\tau) & =p_{\mathrm{I}}+\dot{p}_{\mathrm{I}} \tau+\frac{1}{2} \ddot{p}_{\mathrm{I}} \tau^{2}+\frac{1}{6} \dddot{p}_{\mathrm{I}} \tau^{3}+\cdots, \\
\mathbf{u}_{j}(\tau) & =\mathbf{u}_{j}+\dot{\mathbf{u}}_{j} \tau+\frac{1}{2} \ddot{\mathbf{u}}_{j} \tau^{2}+\frac{1}{6} \dddot{\mathbf{u}}_{j} \tau^{3}+\cdots
\end{aligned}
$$

where

$$
()^{\cdot}=\frac{\partial()}{\partial \tau}=\frac{\partial()}{\partial p_{\mathrm{II}}}, \quad \text { and } \quad \dot{p}_{\mathrm{II}}=\frac{\partial p_{\mathrm{II}}}{\partial p_{\mathrm{II}}}=1, \quad \ddot{p}_{\mathrm{II}}=\dddot{p}_{\mathrm{II}}=\cdots=0 .
$$


Equations (5.33), (5.44) and (5.46) can be used for finding the unknown first derivatives. In matrix notation they have the following form:

$$
\left[\begin{array}{ccc}
\mathbf{K}_{i j} & 0 & -\mathbf{g}_{\mathrm{I} i} \\
\mathbf{L}_{i k j} \mathbf{u}_{k} & \mathbf{K}_{i j} & -\mathbf{K}_{\mathrm{I} i j}^{\mathrm{C}} \mathbf{u}_{j} \\
0 & \mathbf{u}_{j}^{\mathrm{T}} & 0
\end{array}\right]\left[\begin{array}{c}
\dot{\mathbf{t}}_{j} \\
\dot{\mathbf{u}}_{j} \\
\dot{p}_{\mathrm{I}}
\end{array}\right]=-\left[\begin{array}{c}
-\mathbf{g}_{\mathrm{II} i} \\
-\mathbf{K}_{\mathrm{II} i j}^{\mathrm{C}} \mathbf{u}_{j j} \\
0
\end{array}\right],
$$

Equations (5.34), (5.45) and (5.47) provide the second derivatives. Their matrix form is given by

$$
\begin{gathered}
=\left[\begin{array}{ccc}
\mathbf{K}_{i j} & 0 & -\mathbf{g}_{\mathrm{I} i} \\
\mathbf{L}_{i k j} \mathbf{u}_{k} & \mathbf{K}_{i j} & -\mathbf{K}_{\mathrm{I} i j}^{\mathrm{C}} \mathbf{u}_{j} \\
0 & \mathbf{u}_{j}^{\mathrm{T}} & 0
\end{array}\right]\left[\begin{array}{c}
\ddot{\mathbf{t}}_{j} \\
\ddot{\mathbf{u}}_{j} \\
\ddot{p}_{\mathrm{I}}
\end{array}\right]= \\
=\left[\begin{array}{c}
\mathbf{L}_{i k j} \dot{\mathbf{t}}_{k} \dot{\mathbf{t}}_{j}-2 \mathbf{K}_{\mathrm{I} i j}^{\mathrm{C}} \dot{\mathbf{t}}_{j} \dot{p}_{\mathrm{I}}-2 \mathbf{K}_{\mathrm{II} i j}^{\mathrm{C}} \dot{\mathbf{t}}_{j} \\
\mathbf{N}_{i l k j} \dot{\mathbf{t}}_{l} \dot{\mathbf{t}}_{k} \mathbf{u}_{j}-2 \mathbf{M}_{\mathrm{I} i k j} \dot{\mathbf{t}}_{k} \mathbf{u}_{j} \dot{p}_{\mathrm{I}}-2 \mathbf{M}_{\mathrm{II} i k j} \dot{\mathbf{t}}_{k} \mathbf{u}_{j}+2 \mathbf{L}_{i k j} \dot{\mathbf{t}}_{k} \dot{\mathbf{u}}_{j}-2 \mathbf{K}_{\mathrm{I} i j}^{\mathrm{C}} \dot{p}_{\mathrm{I}} \dot{\mathbf{u}}_{j}-2 \mathbf{K}_{\mathrm{II} i j}^{\mathrm{C}} \dot{p}_{\mathrm{II}} \dot{\mathbf{u}}_{j} \\
\dot{\mathbf{u}}_{j}^{\mathrm{T}} \dot{\mathbf{u}}_{j}
\end{array}\right] .
\end{gathered}
$$

5.2.2.2. Second, we look for the equilibrium state $\left(\bar{B}^{\mathrm{cr}}+\Delta \bar{B}^{\mathrm{cr}}\right)$ by selecting a displacement component for the governing parameter $\left(\tau=t_{m}-t_{m 0}, \Delta \tau=\Delta t_{m}\right)$. Then

$$
\begin{aligned}
\mathbf{t}_{\alpha}(\tau) & =\mathbf{t}_{\alpha}+\dot{\mathbf{t}}_{\alpha} \tau+\frac{1}{2} \ddot{\mathbf{t}}_{\alpha} \tau^{2}+\frac{1}{6} \dddot{\mathbf{t}}_{\alpha} \tau^{3}+\cdots, \\
p_{\mathrm{I}}(\tau) & =p_{\mathrm{I}}+\dot{p}_{\mathrm{I}} \tau+\frac{1}{2} \ddot{p}_{\mathrm{I}} \tau^{2}+\frac{1}{6} \dddot{p}_{\mathrm{I}} \tau^{3}+\cdots, \\
p_{\mathrm{II}}(\tau) & =p_{\mathrm{II}}+\dot{p}_{\mathrm{I}} \tau+\frac{1}{2} \ddot{p}_{\mathrm{II}} \tau^{2}+\frac{1}{6} \dddot{p}_{\mathrm{II}} \tau^{3}+\cdots, \\
\mathbf{u}_{j}(\tau) & =\mathbf{u}_{j}+\dot{\mathbf{u}}_{j} \tau+\frac{1}{2} \ddot{\mathbf{u}}_{j} \tau^{2}+\frac{1}{6} \dddot{\mathbf{u}}_{j} \tau^{3}+\cdots
\end{aligned}
$$

where

$$
()^{\cdot}=\frac{\partial()}{\partial \tau}=\frac{\partial()}{\partial t_{m}} \quad \text { and } \quad \dot{t}_{m}=\frac{\partial t_{m}}{\partial t_{m}}=1, \quad \ddot{t}_{m}=\dddot{t}_{m}=\cdots=0 .
$$

Equations 5.33, 5.44 and 5.46 make it possible to determine the first derivatives. They can be given in a matrix form as well:

$$
\left[\begin{array}{cccc}
\mathbf{K}_{i \beta} & 0 & -\mathbf{g}_{\mathrm{I} i} & -\mathbf{g}_{\mathrm{II} i} \\
\mathbf{L}_{i k \beta} \mathbf{u}_{k} & \mathbf{K}_{i j} & -\mathbf{K}_{\mathrm{I} i k}^{\mathrm{C}} \mathbf{u}_{k} & -\mathbf{K}_{\mathrm{II} i k}^{\mathrm{C}} \mathbf{u}_{k} \\
0 & \mathbf{u}_{\underline{\mathbf{s}} j}^{\mathrm{T}} & 0 & 0
\end{array}\right]\left[\begin{array}{c}
\dot{\mathbf{t}}_{\beta} \\
\dot{\mathbf{u}}_{j} \\
\dot{p}_{\mathrm{I}} \\
\dot{p}_{\mathrm{II}}
\end{array}\right]=-\left[\begin{array}{c}
\mathbf{k}_{i m} \\
\mathbf{L}_{i k m} \mathbf{u}_{k} \\
0
\end{array}\right]
$$


As regards the second derivatives (5.34), (5.45) and (5.47) yield the following matrix equation

$$
\begin{gathered}
-\left[\begin{array}{cccc}
\mathbf{K}_{i \beta} & 0 & -\mathbf{g}_{\mathrm{I} i} & -\mathbf{g}_{\mathrm{II} i} \\
\mathbf{L}_{i k \beta} \mathbf{u}_{k} & \mathbf{K}_{i j} & -\mathbf{K}_{\mathrm{I} i k}^{\mathrm{C}} \mathbf{u}_{k} & -\mathbf{K}_{\mathrm{II} i k}^{\mathrm{C}} \mathbf{u}_{k} \\
0 & \mathbf{u}_{\underline{\mathrm{s}} \beta}^{\mathrm{T}} & 0 & 0
\end{array}\right]\left[\begin{array}{c}
\ddot{\mathbf{t}}_{\beta} \\
\ddot{\mathbf{u}}_{j} \\
\ddot{p}_{\mathrm{I}} \\
\ddot{p}_{\mathrm{II}}
\end{array}\right]= \\
=\left[\begin{array}{c}
\mathbf{L}_{i k j} \dot{\mathbf{t}}_{k} \dot{\mathbf{t}}_{j}-2 \mathbf{K}_{\mathrm{I} i j}^{\mathrm{C}} \dot{\mathbf{t}}_{j} \dot{p}_{\mathrm{I}}-2 \mathbf{K}_{\mathrm{II} i j}^{\mathrm{C}} \dot{\mathbf{t}}_{j} \\
\mathbf{N}_{i l k j} \dot{\mathbf{t}}_{l} \dot{\mathbf{t}}_{k} \mathbf{u}_{j}-2 \mathbf{M}_{\mathrm{I} i k j} \dot{\mathbf{t}}_{k} \mathbf{u}_{j} \dot{p}_{\mathrm{I}}-2 \mathbf{M}_{\mathrm{II} i k j} \dot{\mathbf{t}}_{k} \mathbf{u}_{j}+2 \mathbf{L}_{i k j} \dot{\mathbf{t}}_{k} \dot{\mathbf{u}}_{j}-2 \mathbf{K}_{\mathrm{I} i j}^{\mathrm{C}} \dot{p}_{\mathrm{I}} \dot{\mathbf{u}}_{j}-2 \mathbf{K}_{\mathrm{II} i j}^{\mathrm{C}} \dot{p}_{\mathrm{II}} \dot{\mathbf{u}}_{j}
\end{array}\right] . \\
\dot{\mathbf{u}}_{j}^{\mathrm{T}} \dot{\mathbf{u}}_{j}
\end{gathered}
$$

It is characteristic of the asymptotic method that the left sides of the corresponding matrix equations are the same independent of the fact what quantity (load or a displacement component) is selected for the governing parameter.

Further matrix equations can be devised for the higher (third, fourth etc.) derivatives to attain the desired accuracy.

5.3. Fold line of the limit points for a geometrical imperfection. On the limit point fold line of geometrical imperfection $p_{\mathrm{II}}=p_{\mathrm{IIO}}=$ constant and $h$ is the imperfection parameter.

In this case equations $(5.1),(5.2),(5.3)$ are also satisfied in state $\left(\bar{B}^{\text {cr }}\right)$ of the body and in the same manner equations (5.4), (5.5), (5.6) should be satisfied in state $\left(\bar{B}^{\mathrm{cr}}+\Delta \bar{B}^{\mathrm{kr}}\right)$.

\subsubsection{Solution by the Newton-Rapson iteration algorithm.}

5.3.1.1. First, the geometrical imperfection parameter is selected for governing parameter $\left(\tau=h-h_{0}, \Delta \tau=\Delta h\right)$. Solutions to equations (5.4)-(5.6) are sought in the form of the series

$$
\begin{aligned}
\mathbf{t}_{j}+\Delta \mathbf{t}_{j} & =\mathbf{t}_{j}+\Delta \mathbf{t}_{1 j}+\Delta \mathbf{t}_{2 j}+\cdots+\Delta \mathbf{t}_{\underline{\underline{s}} j}+\cdots \\
p_{\mathrm{I}}+\Delta p_{\mathrm{I}} & =p_{\mathrm{I}}+\Delta p_{\mathrm{I} \underline{1}}+\Delta p_{\mathrm{I} \underline{2}}+\cdots+\Delta p_{\mathrm{I \underline {s }}}+\cdots, \\
\mathbf{u}_{j}+\Delta \mathbf{u}_{j} & =\mathbf{u}_{j}+\Delta \mathbf{u}_{\underline{1} j}+\Delta \mathbf{u}_{\underline{2} j}+\cdots+\Delta \mathbf{u}_{\underline{\mathbf{s}} j}+\cdots
\end{aligned}
$$

constituted by the increments $\Delta \mathbf{t}_{\underline{\mathrm{s}} j}, \Delta p_{\mathrm{II}}, \Delta \mathbf{u}_{\underline{\mathrm{s}} j} ; \underline{\mathbf{s}}=\underline{1}, \underline{2}, \cdots,\left(h=h_{0}, \Delta h=\right.$ constant).

Equations (5.4)-(5.6) can now be linearized in the following manner $\left(p_{\mathrm{II}}=p_{\mathrm{II} 0}=\right.$ constant, $\left.\Delta p_{\mathrm{II}}=0\right)$ :

$$
\begin{gathered}
\mathbf{A}_{i}+\frac{\partial \mathbf{A}_{i}}{\partial \mathbf{t}_{j}} \Delta \mathbf{t}_{j}+\frac{\partial \mathbf{A}_{i}}{\partial h} \Delta h-\mathbf{g}_{\mathrm{I} i} \Delta p_{\mathrm{I}}=0 \\
\mathbf{K}_{i j} \mathbf{u}_{j}+\frac{\partial \mathbf{K}_{i j}}{\partial \mathbf{t}_{k}} \Delta \mathbf{t}_{k} \mathbf{u}_{j}+\frac{\partial \mathbf{K}_{i j}}{\partial p_{\mathrm{I}}} \mathbf{u}_{j} \Delta p_{\mathrm{I}}+\frac{\partial \mathbf{K}_{i j}}{\partial h} \mathbf{u}_{j} \Delta h+\mathbf{K}_{i j} \Delta \mathbf{u}_{j}=0
\end{gathered}
$$




$$
\mathbf{u}_{j}^{\mathrm{T}} \Delta \mathbf{u}_{j}=0 \text {. }
$$

In view of relation $(5.36),(5.38)$ ) and the notational convention

$$
\stackrel{*}{()}=\frac{\partial()}{\partial h}
$$

applied in the sequel, the equations that are valid for the $\underline{s}^{\text {th }}$ iteration step can be gathered into a single matrix equation:

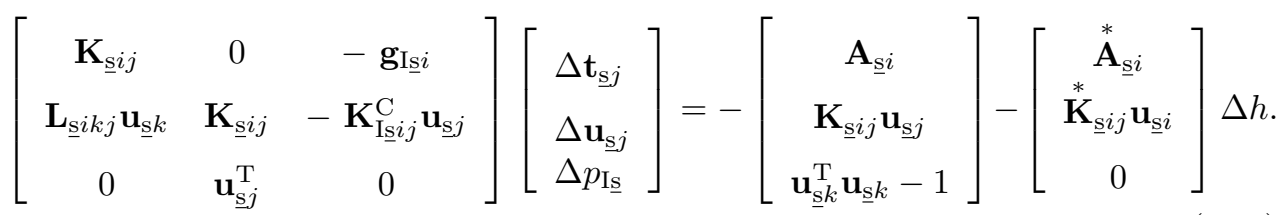

Under a given $\Delta h$ equation system $(5.68)$ is a linear one for calculating $\Delta \mathbf{t}_{\mathrm{s} j}, \Delta \mathbf{u}_{\mathrm{s} j}$, and $\Delta p_{\text {Is }}$. The iteration can be continued till the prescribed error limit is reached.

5.3.1.2. Second, a displacement component is selected as a governing parameter for solving equation system (5.4)-(5.6) $\left(\tau=t_{m}-t_{m 0}, \Delta \tau=\Delta t_{m}\right)$. The solution is sought in the form of the following series

$$
\begin{aligned}
\mathbf{t}_{\alpha}+\Delta \mathbf{t}_{\alpha} & =\mathbf{t}_{\alpha}+\Delta \mathbf{t}_{\underline{1} \alpha}+\Delta \mathbf{t}_{\underline{2} \alpha}+\cdots+\Delta \mathbf{t}_{\underline{\mathrm{s}} \alpha}+\cdots, \quad \alpha=1,2, \ldots, m-1 \\
p_{\mathrm{I}}+\Delta p_{\mathrm{I}} & =p_{\mathrm{I}}+\Delta p_{\mathrm{I} \underline{1}}+\Delta p_{\mathrm{I} \underline{2}}+\cdots+\Delta p_{\mathrm{I} \underline{\mathrm{s}}}+\cdots, \\
h+\Delta h & =h+\Delta p h_{\underline{1}}+\Delta h_{\underline{2}}+\cdots+\Delta h_{\underline{\mathrm{s}}}+\cdots, \\
\mathbf{u}_{j}+\Delta \mathbf{u}_{j} & =\mathbf{u}_{j}+\Delta \mathbf{u}_{\underline{1} j}+\Delta \mathbf{u}_{\underline{2} j}+\cdots+\Delta \mathbf{u}_{\underline{\mathrm{s}} j}+\cdots
\end{aligned}
$$

constituted by the increments $\Delta \mathbf{t}_{\underline{\mathrm{s}} \alpha}, \Delta p_{\mathrm{Is}}, \Delta h_{\underline{\mathrm{s}}}, \Delta \mathbf{u}_{\underline{\mathrm{s}} j} ; \underline{\mathrm{s}}=\underline{1}, \underline{2}, \ldots ;\left(t_{m}=t_{m 0}=\right.$ constant, $\Delta t_{m}=$ constant, $\left.p_{\mathrm{II}}=p_{\mathrm{IIO}}, \Delta p_{\mathrm{II}}=0\right)$.

The linearized form of equations $(5.4)-(5.6)$ is given by:

$$
\begin{gathered}
\mathbf{A}_{i}+\frac{\partial \mathbf{A}_{i}}{\partial \mathbf{t}_{\beta}} \Delta \mathbf{t}_{\beta}+\frac{\partial \mathbf{A}_{i}}{\partial t_{m}} \Delta t_{m}+\frac{\partial \mathbf{A}_{i}}{\partial h} \Delta h-\mathbf{g}_{\mathrm{I} i} \Delta p_{\mathrm{I}}=0 \\
\mathbf{K}_{i j} \mathbf{u}_{j}+\frac{\partial \mathbf{K}_{i j}}{\partial \mathbf{t}_{\beta}} \mathbf{u}_{j} \Delta \mathbf{t}_{\beta}+\frac{\partial \mathbf{K}_{i j}}{\partial t_{m}} \mathbf{u}_{j} \Delta t_{m}+\frac{\partial \mathbf{K}_{i j}}{\partial h} \mathbf{u}_{j} \Delta h+\frac{\partial \mathbf{K}_{i j}}{\partial p_{\mathrm{I}}} \mathbf{u}_{j} \Delta p_{\mathrm{I}}+\mathbf{K}_{i j} \Delta \mathbf{u}_{j}=0 \\
\mathbf{u}_{j}^{\mathrm{T}} \Delta \mathbf{u}_{j}=0 .
\end{gathered}
$$

By taking relations (5.36) and (5.38) into account the equation to be solved in the $\underline{s}^{\text {th }}$ iteration step is of form

$$
\left[\begin{array}{cccc}
\mathbf{K}_{\underline{\mathbf{s}} i \beta} & 0 & -\mathbf{g}_{\underline{I} \underline{\mathbf{s}} i} & -\stackrel{\mathbf{A}}{\underline{\mathbf{s}} i}^{*} \\
\mathbf{L}_{\underline{\mathbf{s}} i k \beta} \mathbf{u}_{\underline{\mathbf{s}} k} & \mathbf{K}_{\underline{\mathbf{s}} i j} & -\mathbf{K}_{\mathrm{I} \underline{\mathbf{s}} i k}^{\mathrm{C}} \mathbf{u}_{\underline{\mathbf{s}} k} & -\mathbf{K}_{\underline{\mathbf{s}} i k} \mathbf{u}_{\underline{\mathbf{s}} k} \\
0 & \mathbf{u}_{\underline{\mathbf{s}} j}^{\mathrm{T}} & 0 & 0
\end{array}\right]\left[\begin{array}{c}
\Delta \mathbf{t}_{\underline{\mathbf{s}} \beta} \\
\Delta \mathbf{u}_{\underline{\mathbf{s}} j} \\
\Delta p_{\underline{I} \underline{\mathbf{s}}} \\
\Delta h_{\underline{\mathbf{s}}}
\end{array}\right]=
$$




$$
=-\left[\begin{array}{c}
\mathbf{A}_{\underline{\underline{s}} i} \\
\mathbf{K}_{\underline{\underline{s}} i j} \mathbf{u}_{\underline{\mathbf{s}} j} \\
\mathbf{u}_{\underline{\underline{s}} k}^{\mathrm{T}} \mathbf{u}_{\underline{\mathbf{s}} k}-1
\end{array}\right]-\left[\begin{array}{c}
\mathbf{k}_{\underline{\underline{s}} i m} \\
\mathbf{L}_{\underline{\mathbf{s}} i k m} \mathbf{u}_{\underline{\mathbf{s}} k} \\
0
\end{array}\right] \Delta t_{m}
$$

in matrix notation. If $\Delta t_{m}$ is given $\Delta \mathbf{t}_{\underline{\mathbf{s}} \alpha}, \Delta p_{\underline{I}}, \Delta h_{\underline{\mathbf{s}}}$ and $\Delta \mathbf{u}_{\underline{\mathbf{s}} j}$ are the unknowns in the above linear equitation system. The iteration should be continued till the prescribed error limit is satisfied.

5.3.2. Solution by the asymptotic numerical method. For applying the asymptotic numerical method it is worth gathering the corresponding equilibrium equations on the basis of Subsection $5.2 .2\left(p_{\mathrm{II}}=p_{\mathrm{II} O}=\right.$ constant, $\dot{p}_{\mathrm{II}}=\ddot{p}_{\mathrm{II}}=\cdots=0$ !). Regarding equilibrium equation (5.32) as a point of departure we get

$$
\dot{\mathbf{A}}_{i}=\mathbf{K}_{i j} \dot{\mathbf{t}}_{j}+\stackrel{\mathbf{A}}{i}_{i} \dot{h}-\mathbf{g}_{\mathrm{I} i} \dot{p}_{\mathrm{I}}=0,
$$

$$
\ddot{\mathbf{A}}_{i}=\mathbf{K}_{i j} \ddot{\mathbf{t}}_{j}-\mathbf{g}_{\mathrm{I} i} \ddot{p}_{\mathrm{I}}+\stackrel{*}{\mathbf{A}_{i}} \ddot{h}+\mathbf{L}_{i k j} \dot{\mathbf{t}}_{k} \dot{\mathbf{t}}_{j}-2 \mathbf{K}_{\mathrm{I} i j}^{\mathrm{C}} \dot{\mathbf{t}}_{j} \dot{p}_{\mathrm{I}}+2 \stackrel{\mathbf{K}}{i j}^{*} \dot{\mathbf{t}}_{j} \dot{h}-2 \stackrel{\mathbf{g}}{\mathrm{I} i}^{*} \dot{p}_{\mathrm{I}} \dot{h}+\stackrel{\mathbf{A}}{i}_{i} \dot{h}^{2}=0,
$$

$$
\begin{aligned}
& \dddot{\mathbf{A}}_{i}=\mathbf{K}_{i j} \dddot{\mathbf{t}}_{j}-\mathbf{g}_{\mathrm{I} i} \dddot{p}_{\mathrm{I}}+3 \mathbf{L}_{i k j} \dot{\mathbf{t}}_{k} \ddot{\mathbf{t}}_{j}-3 \mathbf{K}_{\mathrm{I} i j}^{\mathrm{C}} \ddot{\mathbf{t}}_{j} \dot{p}_{\mathrm{I}}-3 \mathbf{K}_{\mathrm{I} i j}^{\mathrm{C}} \dot{\mathbf{t}}_{j} \ddot{p}_{\mathrm{I}}+\mathbf{N}_{i l k j} \dot{\mathbf{t}}_{l} \dot{\mathbf{t}}_{k} \dot{\mathbf{t}}_{j}- \\
& -3 \mathbf{M}_{\mathrm{I} i k j} \dot{\mathbf{t}}_{k} \dot{\mathbf{t}}_{j} \dot{p}_{\mathrm{I}}++3 \stackrel{*}{\mathbf{K}_{i j}} \ddot{\mathbf{t}}_{j} \dot{h}+3 \stackrel{*}{\mathbf{K}_{i j}} \dot{\mathbf{t}}_{j} \ddot{h}-3 \stackrel{*}{\mathrm{~g}}_{\mathrm{I} i} \ddot{p}_{\mathrm{I}} \dot{h}-3 \stackrel{*}{\mathbf{g}}_{\mathrm{I} i} \dot{p}_{\mathrm{I}} \ddot{h}+3 \stackrel{*}{\mathbf{L}}_{i k j} \dot{\mathbf{t}}_{k} \dot{\mathbf{t}}_{j} \dot{h}- \\
& -6 \stackrel{*}{\mathbf{K}}_{\mathrm{I} i j}^{C} \dot{\mathbf{t}}_{j} \dot{p}_{\mathrm{I}} \dot{h}+3 \stackrel{* *}{\mathbf{K}}_{i j} \dot{\mathbf{t}}_{j} \dot{h}^{2}-3 \mathbf{g}_{\mathrm{I} i}^{* *} \dot{p}_{\mathrm{I}} \dot{h}^{2}+3 \mathbf{A}_{i} \ddot{h} \dot{h}+\stackrel{* * *}{\mathbf{A}_{i}} \dot{h}^{3}=0 .
\end{aligned}
$$

Determine now the derivatives of equations 5.2$)_{1}$ and 5.3 :

$$
\begin{aligned}
& \left(\mathbf{K}_{i j} \mathbf{u}_{j}\right)^{\cdot}=\mathbf{L}_{i k j} \mathbf{u}_{j} \dot{\mathbf{t}}_{k}-\mathbf{K}_{\mathrm{I} i j}^{\mathrm{C}} \mathbf{u}_{j} \dot{p}_{\mathrm{I}}+\stackrel{\mathbf{K}}{i j}^{*} \mathbf{u}_{j} \dot{h}+\mathbf{K}_{i j} \dot{\mathbf{u}}_{j}=0,
\end{aligned}
$$

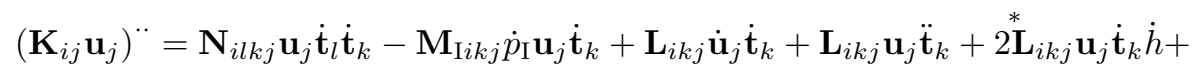

$$
\begin{aligned}
& +\stackrel{*}{\mathbf{K}}_{i j} \mathbf{u}_{j} \dot{h}^{2}-\mathbf{M}_{\mathrm{I} k i j} \mathbf{u}_{j} \dot{\mathbf{t}}_{k} \dot{p}_{\mathrm{I}}-2 \stackrel{*}{\mathbf{K}_{\mathrm{I} i j}^{C}} \mathbf{u}_{j} \dot{p}_{\mathrm{I}} \dot{h}+\mathbf{K}_{\mathrm{I} i j}^{\mathrm{C}} \mathbf{u}_{j} \ddot{p}_{\mathrm{I}}-\mathbf{K}_{\mathrm{I} i j}^{\mathrm{C}} \dot{\mathbf{u}}_{j} \dot{p}_{\mathrm{I}}+2 \stackrel{*}{\mathbf{K}}_{i j} \dot{\mathbf{u}}_{j} \dot{h}+ \\
& +\stackrel{*}{\mathbf{K}_{i j}} \mathbf{u}_{j} \ddot{h}+\mathbf{L}_{i k j} \dot{\mathbf{u}}_{j} \dot{\mathbf{t}}_{k}-\mathbf{K}_{\mathrm{I} i j}^{\mathrm{C}} \dot{p}_{\mathrm{I}} \dot{\mathbf{u}}_{j}+\mathbf{K}_{i j} \ddot{\mathbf{u}}_{j}=0, \\
& \mathbf{u}_{j}^{\mathrm{T}} \dot{\mathbf{u}}_{j}=0 \\
& \dot{\mathbf{u}}_{j}^{\mathrm{T}} \dot{\mathbf{u}}_{j}+\mathbf{u}_{j}^{\mathrm{T}} \ddot{\mathbf{u}}_{j}=0 \text {. }
\end{aligned}
$$

5.3.2.1. First, let us determine the equilibrium state $\left(\bar{B}^{\text {cr }}+\Delta \bar{B}^{\text {cr }}\right)$ by using the geometrical imperfection parameter as governing parameter in the following manner $\left(\tau=h-h_{0}, \Delta \tau=\Delta h\right)$

$$
\begin{aligned}
& \mathbf{t}_{i}(\tau)=\mathbf{t}_{i}+\dot{\mathbf{t}}_{i} \tau+\frac{1}{2} \ddot{\mathbf{t}}_{i} \tau^{2}+\frac{1}{6} \dddot{\mathbf{t}}_{i} \tau^{3}+\cdots, \\
& p_{\mathrm{I}}(\tau)=p_{\mathrm{I}}+\dot{p}_{\mathrm{I}} \tau+\frac{1}{2} \ddot{p}_{\mathrm{I}} \tau^{2}+\frac{1}{6} \dddot{p}_{\mathrm{I}} \tau^{3} \cdots, \\
& \mathbf{u}_{j}(\tau)=\mathbf{u}_{j}+\dot{\mathbf{u}}_{j} \tau+\frac{1}{2} \ddot{\mathbf{u}}_{j} \tau^{2}+\frac{1}{6} \dddot{\mathbf{u}}_{j} \tau^{3}+\cdots
\end{aligned}
$$


where

$$
()^{\cdot}=\frac{\partial()}{\partial \tau}=\frac{\partial()}{\partial h}, \quad \text { and } \quad \dot{h}=\frac{\partial h}{\partial h}=1, \quad \ddot{h}=\dddot{h}=\cdots=0 .
$$

Equations (5.77), (5.80) and 5.82) set up for the first derivatives can be given in matrix notation as well:

$$
\left[\begin{array}{ccc}
\mathbf{K}_{i j} & 0 & -\mathbf{g}_{\mathrm{I} i} \\
\mathbf{L}_{i k j} \mathbf{u}_{k} & \mathbf{K}_{i j} & -\mathbf{K}_{\mathrm{I} i j}^{\mathrm{C}} \mathbf{u}_{j} \\
0 & \mathbf{u}_{j}^{\mathrm{T}} & 0
\end{array}\right]\left[\begin{array}{c}
\dot{\mathbf{t}}_{j} \\
\dot{\mathbf{u}}_{j} \\
\dot{p}_{\mathrm{I}}
\end{array}\right]=-\left[\begin{array}{c}
\mathbf{A}_{i}^{*} \\
\mathbf{K}_{i j} \mathbf{u}_{j} \\
0
\end{array}\right]
$$

As regards the second derivatives the matrix form of equilibrium equations 5.78, (5.81) and (5.83) is as follows:

$$
\begin{aligned}
& {\left[\begin{array}{ccc}
\mathbf{K}_{i j} & 0 & -\mathbf{g}_{\mathrm{I} i} \\
\mathbf{L}_{i k j} \mathbf{u}_{k} & \mathbf{K}_{i j} & -\mathbf{K}_{\mathrm{I} i j}^{\mathrm{C}} \mathbf{u}_{j} \\
0 & \mathbf{u}_{j}^{\mathrm{T}} & 0
\end{array}\right]\left[\begin{array}{c}
\ddot{\mathbf{t}}_{j} \\
\ddot{\mathbf{u}}_{j} \\
\ddot{p}_{\mathrm{I}}
\end{array}\right]=} \\
& =-\left[\begin{array}{c}
\mathbf{L}_{i k j} \dot{\mathbf{t}}_{k} \dot{\mathbf{t}}_{j}-2 \mathbf{K}_{\mathrm{I} i j}^{\mathrm{C}} \dot{\mathbf{t}}_{j} \dot{p}_{\mathrm{I}}+2 \stackrel{\mathbf{K}}{i j} \dot{\mathbf{t}}_{j}-2 \stackrel{*}{\mathrm{I}}_{\mathrm{I} i} \dot{p}_{\mathrm{I}} \dot{h}+\stackrel{* *}{\mathbf{A}} \\
\mathbf{N}_{i l k j} \dot{\mathbf{t}}_{l} \dot{\mathbf{t}}_{k} \mathbf{u}_{j}-2 \mathbf{M}_{\mathrm{I} i k j} \dot{\mathbf{t}}_{k} \mathbf{u}_{j} \dot{p}_{\mathrm{I}}+2 \mathbf{L}_{i k j} \dot{\mathbf{t}}_{k} \dot{\mathbf{u}}_{j}-2 \mathbf{K}_{\mathrm{I} i j}^{\mathrm{C}} \dot{p}_{\mathrm{I}} \dot{\mathbf{u}}_{j} \\
\dot{\mathbf{u}}_{j}^{\mathrm{T}} \dot{\mathbf{u}}_{j}
\end{array}\right] \\
& -\left[\begin{array}{c}
0 \\
2 \stackrel{\mathbf{L}}{i k j} \mathbf{u}_{j} \dot{\mathbf{t}}_{k}-2 \stackrel{*}{\mathbf{K}}_{\mathrm{I} i j}^{C} \mathbf{u}_{j} \dot{p}_{\mathrm{I}}+2 \stackrel{*}{\mathbf{K}}_{i j} \dot{\mathbf{u}}+\stackrel{*}{\mathbf{K}}_{i j} \mathbf{u}_{j} \\
0
\end{array}\right] .
\end{aligned}
$$

5.3.2.2. Second, it is assumed that the governing parameter for finding the equilibrium state $\left(\bar{B}^{\mathrm{cr}}+\Delta \bar{B}^{\mathrm{cr}}\right)$ is the displacement component $t_{m}\left(\tau=t_{m}-t_{m 0}, \Delta \tau=\right.$ $\left.\Delta t_{m}\right)$. Then

$$
\begin{aligned}
& \mathbf{t}_{\alpha}(\tau)=\mathbf{t}_{\alpha}+\dot{\mathbf{t}}_{\alpha} \tau+\frac{1}{2} \ddot{\mathbf{t}}_{\alpha} \tau^{2}+\frac{1}{6} \dddot{\mathbf{t}}_{\alpha} \tau^{3}+\cdots, \\
& p_{\mathrm{I}}(\tau)=p_{\mathrm{I}}+\dot{p}_{\mathrm{I}} \tau+\frac{1}{2} \ddot{p}_{\mathrm{I}} \tau^{2}+\frac{1}{6} \dddot{p}_{\mathrm{I}} \tau^{3} \cdots, \\
& \mathbf{u}_{j}(\tau)=\mathbf{u}_{j}+\dot{\mathbf{u}}_{j} \tau+\frac{1}{2} \ddot{\mathbf{u}}_{j} \tau^{2}+\frac{1}{6} \dddot{\mathbf{u}}_{j} \tau^{3}+\cdots \\
& h(\tau)=h+\dot{h} \tau+\frac{1}{2} \ddot{h} \tau^{2}+\frac{1}{6} \dddot{h} \tau^{3}+\cdots
\end{aligned}
$$

where

$$
()^{\cdot}=\frac{\partial()}{\partial \tau}=\frac{\partial()}{\partial t_{m}}, \quad \text { and } \quad \dot{t}_{m}=\frac{\partial t_{m}}{\partial t_{m}}=1, \quad \ddot{t}_{m}=\dddot{t}_{m}=\cdots=0 .
$$


In the present case

$$
\left[\begin{array}{cccc}
\mathbf{K}_{i \beta} & 0 & -\mathbf{g}_{\mathrm{I} i} & \stackrel{\mathbf{A}}{i}^{*} \\
\mathbf{L}_{i k \beta} \mathbf{u}_{k} & \mathbf{K}_{i j} & -\mathbf{K}_{\mathrm{I} i k}^{\mathrm{C}} \mathbf{u}_{k} & \stackrel{\mathbf{K}}{i k}_{i k} \mathbf{u}_{k} \\
0 & \mathbf{u}_{\underline{\mathbf{s}} j}^{\mathrm{T}} & 0 & 0
\end{array}\right]\left[\begin{array}{c}
\dot{\mathbf{t}}_{\beta} \\
\dot{\mathbf{u}}_{j} \\
\dot{p}_{\mathrm{I}} \\
\dot{h}
\end{array}\right]=-\left[\begin{array}{c}
\mathbf{k}_{i m} \\
\mathbf{L}_{\alpha k m} \mathbf{u}_{k} \\
0
\end{array}\right],
$$

is the matrix form of equations (5.77), (5.80) and $(5.82)$ derived for the first derivatives. As regards the second derivatives equations (5.78), 5.81) and (5.83) can also be presented in a matrix form:

$$
\begin{aligned}
& {\left[\begin{array}{cccc}
\mathbf{K}_{i \beta} & 0 & -\mathbf{g}_{\mathrm{I} i} & -\mathbf{g}_{\mathrm{II} i} \\
\mathbf{L}_{i k \beta} \mathbf{u}_{k} & \mathbf{K}_{i j} & -\mathbf{K}_{\mathrm{I} i k}^{\mathrm{C}} \mathbf{u}_{k} & \stackrel{\mathbf{K}}{i k}_{i k} \mathbf{u}_{k} \\
0 & \mathbf{u}_{\underline{\mathbf{s}} j}^{\mathrm{T}} & 0 & 0
\end{array}\right]\left[\begin{array}{c}
\ddot{\mathbf{t}}_{\beta} \\
\ddot{\mathbf{u}}_{j} \\
\ddot{p}_{\mathrm{I}} \\
\ddot{h}
\end{array}\right]=} \\
& =-\left[\begin{array}{c}
\mathbf{L}_{i k j} \dot{\mathbf{t}}_{k} \dot{\mathbf{t}}_{j}-2 \mathbf{K}_{\mathrm{I} i j}^{\mathrm{C}} \dot{\mathbf{t}}_{j} \dot{p}_{\mathrm{I}} \\
\mathbf{N}_{i l k j} \dot{\mathbf{t}}_{l} \dot{\mathbf{t}}_{k} \mathbf{u}_{j}-2 \mathbf{M}_{\mathrm{I} i k j} \dot{\mathbf{t}}_{k} \mathbf{u}_{j} \dot{p}_{\mathrm{I}}+2 \mathbf{L}_{i k j} \dot{\mathbf{t}}_{k} \dot{\mathbf{u}}_{j}-2 \mathbf{K}_{\mathrm{I} j}^{\mathrm{C}} \dot{p}_{\mathrm{I}} \dot{\mathbf{u}}_{j} \\
\dot{\mathbf{u}}_{j}^{\mathrm{T}} \dot{\mathbf{u}}_{j}
\end{array}\right]-
\end{aligned}
$$

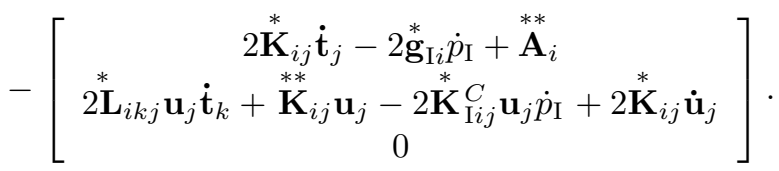

Equations for the third, fourth etc. derivatives can also be derived in case they are needed to attain the required accuracy.

\section{Concluding Remarks}

The present paper investigated the following issues if a 3D body is subjected to a distributed load perpendicular to the deformed surface:

- what the characteristics are of the critical states,

- how to determine the fold line of limit points under various conditions.

In each case a FEM based numerical algorithm has been developed. Unfortunately there are no solved numerical examples at the moment though these could provide the final proof for the effective applicability of the methods presented in the paper. The author truly hopes that his former students shall be able to code the procedures suggested and then they will perform the necessary computations. 


\section{REFERENCES}

1. S. P. Thimosenko and J. M. Gere. Theory of Elastic Stability. United Engineering Trustees, Inc., 1936.

2. V. V. Bolotin. The Dynamic Stability of Elastic Systems. Holdan-Day, Inc., 1964.

3. J. M. T. Thompson and G. W. Hunt. A General Theory of Elastic Stability. John Wiley \& Sons, 1973.

4. K. Huseyin. Nonlinear Theory of Elastic Stability. Ed. by G. Æ. Oravas. Monographs and textbooks on mechanics of solids and fluids. Noordhof International Publishing, Leyden, 1975.

5. K. Huseyin. Multiple Parameter Stability Theory and its Applications: Bifurcations, Catastrophes, Instabilities. Oxford Engineering Science Series (Book 18). Oxford University Press, 1986.

6. L. A. Godoy. Theory of Elastic Structures - Analysis and Sensitivity. Taylor \& Francis, 1999.

7. N. A. Alfutov. Stability of Elastic Structures. Springer, 2000. DOI: 10.1007/9783-540-49098-2

8. J. R. Millard. Buckling of bars, plates, and shells. Blacksburg, Bull Ridge Publishing, 2006.

9. Zdeněk P. Bažant and Luigi Cedolin. Stability of Structures. First published by Oxford University Press in 1991. Dover Publications, Inc. Mineola, New York, 2003 .

10. Z. Waszczyszyn, Cz. Cichon, and M. Radwanska. Stability of Structures by Finite Element Methods. Studies in Applied Mechanics (Book 40). Elsevier Science, 1994.

11. F. G. Flores and L. A. Godoy. "Elastic postbuckling analysis via finite element and perturbation techniques. Part 1, Formulation." International Journal for Numerical Methods in Engineering, 33, (1992), pp. 1775-1794. DOI: 10.1002/ nme.1620330902,

12. F. G. Flores and L. A. Godoy. "Elastic postbuckling analysis via finite element and perturbation techniques. Part 2, Application to shells of revolution." International Journal for Numerical Methods in Engineering, 33, (1993), pp. 331354. DOI: $10.1002 / \mathrm{nme} .1620330902$.

13. C. Trusell and W. Noll. The Non-Linear Field Theories of Mechanics. Second. Springer-Verlag Berlin Heidelberg, 1992. DOI: 10.1007/978-3-662-10388-3. 